University of Louisville

ThinkIR: The University of Louisville's Institutional Repository

Electronic Theses and Dissertations

$11-2011$

\title{
A statin-regulated microRNA represses c-Myc expression and function.
}

Apana Agha L. Takwi 1980-

University of Louisville

Follow this and additional works at: https://ir.library.louisville.edu/etd

\section{Recommended Citation}

Takwi, Apana Agha L. 1980-, "A statin-regulated microRNA represses c-Myc expression and function." (2011). Electronic Theses and Dissertations. Paper 1415.

https://doi.org/10.18297/etd/1415

This Doctoral Dissertation is brought to you for free and open access by ThinkIR: The University of Louisville's Institutional Repository. It has been accepted for inclusion in Electronic Theses and Dissertations by an authorized administrator of ThinkIR: The University of Louisville's Institutional Repository. This title appears here courtesy of the author, who has retained all other copyrights. For more information, please contact thinkir@louisville.edu. 


\title{
A STATIN-REGULATED MICRORNA REPRESSES $C$-MYC EXPRESSION AND FUNCTION
}

\author{
By
}

Apana Agha L. Takwi

B.Sc., University of Buea, 2003

\begin{abstract}
A Dissertation
Submitted to the Faculty of the

School of Medicine of the University of Louisville

in Partial Fulfillment of the Requirements

for the Degree of
\end{abstract}

Doctor of Philosophy

Department of Biochemistry and Molecular Biology

University of Louisville

Louisville, Kentucky

December 2011 

FUNCTION

By

Apana Agha L. Takwi

B.Sc. University of Buea, 2003

A Dissertation Approved on

November 1, 2011

By the following Dissertation Committee:

Dissertation Director: Dr. Yong Li

Dr. Russell Prough

Dr. Tom Geoghegan

Dr. Seven Ellis

Dr. Daniel Conklin 


\section{DEDICATION}

This dissertation is dedicated to my parents, Mr. and Mrs. Takwi

who have given me invaluable support and educational

opportunities over the years 


\section{ACKOWLEDGEMENTS}

I would like to thank my mentor, Dr. Yong Li, for his guidance and patience. I would also like to thank my dissertation committee members, Dr. Russell Prough, Dr. Tom Geoghegan, Dr. Steven Ellis and Dr. Daniel Conklin, for their comments and assistance over the past three years. I would like to express my thanks to my wife, Lilian, for her understanding and patience during the numerous hard and

trying times. Also many thanks to my family: Lilian, Roy, Kenneth, Jacky, Judith, Marlouise, Rosita and Anih Takwi. 


\begin{abstract}
A STATIN-REGULATED MICRORNA REPRESSES $C$-MYC EXPRESSION AND FUNCTION

APANA AGHA L. TAKWI

NOVEMBER 1, 2011
\end{abstract}

c-Myc dysregulation is one of the most common abnormalities found in human cancer. MicroRNAs (miRNAs) are functionally intertwined with the c-Myc network as multiple miRNAs are regulated by c-Myc, while others directly suppress c-Myc expression. In this work, we identified miR-33b as a primate-specific negative regulator of c-Myc. The human $m i R-33 b$ gene is located at $17 \mathrm{p} 11.2$, a genomic locus frequently lost in medulloblastomas, which are pediatric brain tumors that display c-Myc overproduction. Through a small-scale screening with drugs approved by the US Food and Drug Administration (FDA), we found that lovastatin up-regulated miR-33b expression, reduced cell proliferation, and impaired c-Myc expression and function in miR-33bpositive medulloblastoma cells. In addition, a low dose of lovastatin treatment at a level comparable to approved human oral use reduced tumor growth in mice orthotopically xenografted with cells carrying $m i R-33 b$, but not with cells lacking $m i R-33 b$. This work presents a highly promising therapeutic option, using drug repurposing and a miRNA as a biomarker, against cancers that over-express c-Myc. 
PAGE

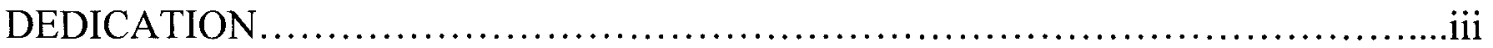

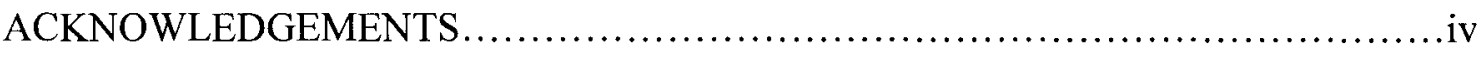

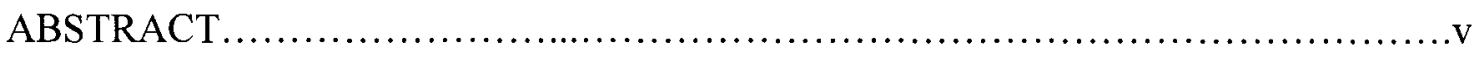

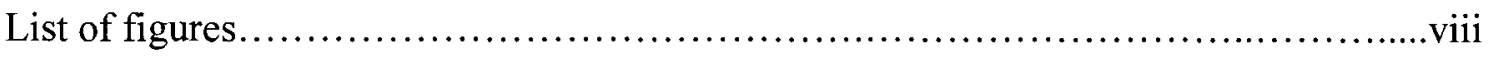

\section{CHAPTER}

1: MICRORNA REGULATION AND MEDULLOBLASTOMA DEVELOPMEN.......1

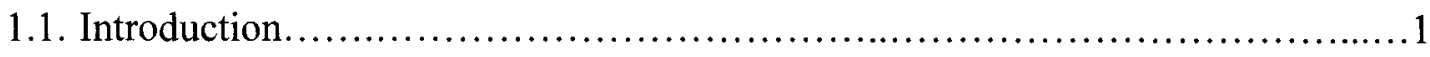

1.2. Childhood Brain tumors............................................................. 1

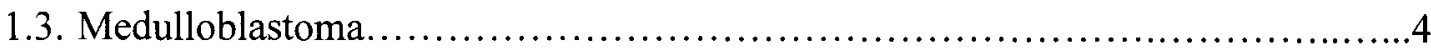

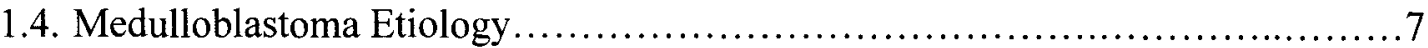

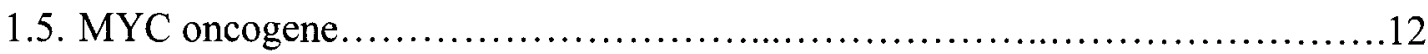

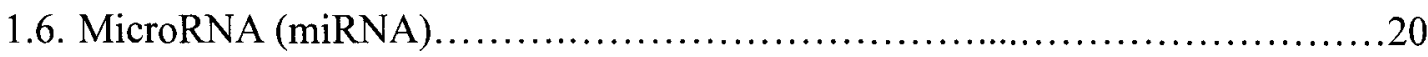

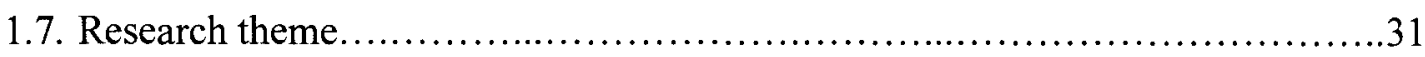

2: MICRORNA-33b (miR-33b) TARGETS MYC AND NEGATIVELY AFFECTS MEDULLOBLASTOMA DEVELOPMENT ....................................3

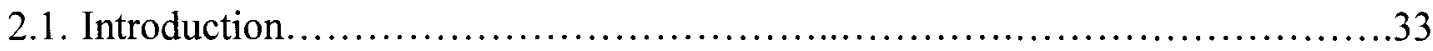

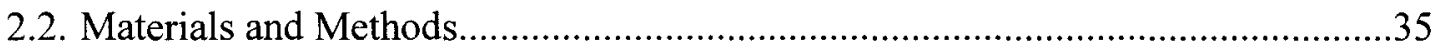

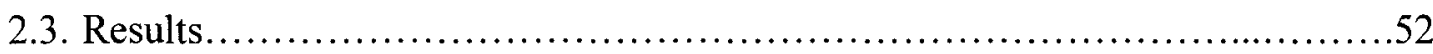

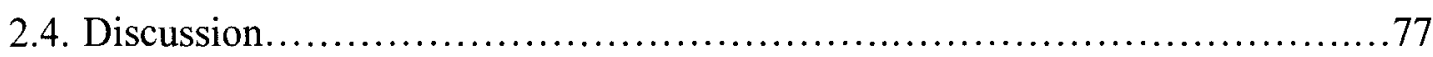


3: A STATIN-REGULATED MICRORNA REPRESSES c-Myc EXPRESSION AND

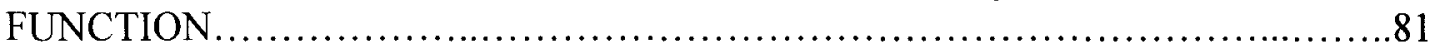

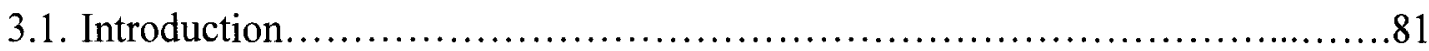

3.2. Materials and Methods......................................................... 83

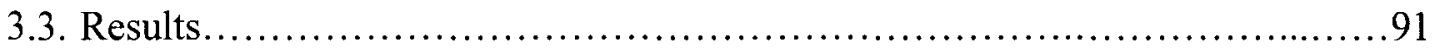

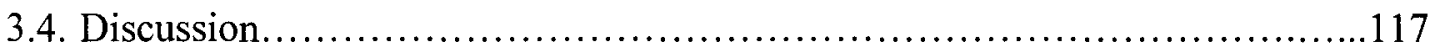

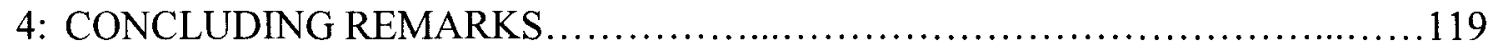

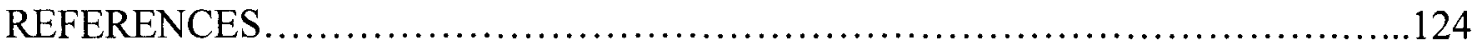

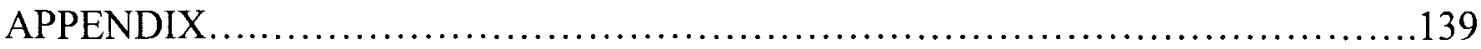

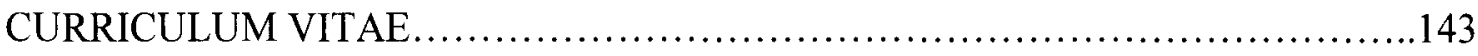




\section{LIST OF FIGURES}

PAGE

\section{FIGURES}

1. Schematic representation of the molecular progression of Medulloblastoma. 6

2. Schematic of the Wnt and Shh signaling pathway........................ 10

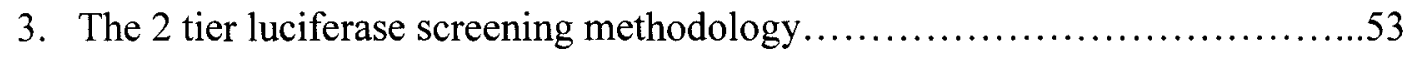

4. $\mathrm{miR}-33 \mathrm{~b}$ is a negative regulator of c-Myc directly targeting the

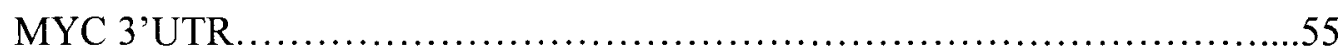

5. Mutate E2F-luc or MYC 3'UTR are not targeted by miR-33b.................56

6. Over-expression of miR-33b results in decreased levels of MYC mRNA.......58

7. Over-expression of miR-33b in $293 \mathrm{~T}$ cells down-regulates c-Myc and

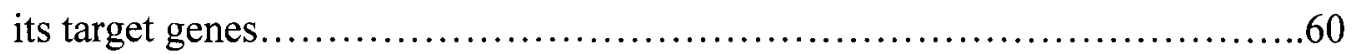

8. c-Myc does not regulate miR-33b expression................................61

9. miR-33b down-regulates exogenously expressed c-Myc in MYC-null

HO15.19 cells.

10. Re-introduction of miR-33b in D283 cells results in negatively regulates c-Myc expression and c-Myc-mediated function.

11. miR-33b negatively affect c-Myc-mediated cell proliferation and migration of D283 cells. 67

12. Stem cell characteristics of D28 cells are adversely affected by miR-33b. 69 
13. Over-expression of miR-33b in Daoy cells down-regulates c-Myc and

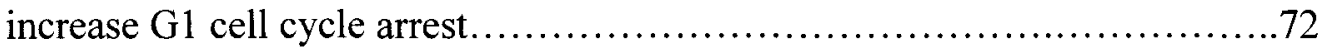

14. miR-33b inhibits c-Myc-mediated cell proliferation and invasiveness of Daoy cells .73

15. miR-33b decreases anchorage-independent colony formation of D283 cells....77

16. miR-33b reintroduction in D283-Med cells reduces tumorigenicity...............76

17. A small scale screening assay to identify FDA-approved compounds that reduce medulloblastoma Daoy cell viability and increase miR-33b expression .92

18. Lovastatin dose-dependent decrease in Daoy, but not D283, cell proliferation..93

19. Lovastatin positively modulates miR-33b expression in miR-33b-positive medulloblastoma cells Daoy and UW228 ...............................95

20. Lovastatin increases miR-33b transcription through the cholesterol synthetic pathway..... .96

21. Impact on cell proliferation and miR-33b expression by other statins in Daoy cells.

22. Lovastatin down-regulates the protein expression of c-Myc and its target in miR-33b-positive medulloblastoma Daoy and UW228 cells

23. Lovastatin increase G1 cell cycle arrest in Daoy, but not D283, cells.

24. Lovastatin decrease cell invasiveness of miR-33b-positive medulloblastoma Daoy cells .101

25. Xenograft models of medulloblastoma with lovastatin treatment. .103

26. Lovastatin treatment led to miR-33b up-regulation and lowered expression of miR-9, c-Myc, and Cyclin E in Daoy tumors

27. HeLa cells show reduced sensitivity in response to lovastatin treatment. 106

28. Expression of miR-33b is lower in HeLa cells compared to Daoy cells in response to FDA approved drugs. 
29. 5-Azacytidine sensitizes HeLa cells to lovastatin-induced inhibition of cell proliferation

30. 5-Azacytidine treatment decreases the methylation level of the SREBFI miR-33b gene enable lovastatin-mediated G1 cell cycle arrest of HeLa cells....110

31. Modulation of miR-33b expression in HeLa cells reciprocally modulates c-Myc protein and MYC mRNA expression

32. Over-expression of miR-33b down-regulated c-Myc-mediated functions in HeLa cells

33. Modulation of miR-33b inversely correlates with HeLa cells invasiveness......115

34. miR-33b-induced apoptosis is rescued by exogenous c-Myc expression 116

35. Proposed schematic of lovastatin induce inhibition of medulloblastoma cell growth 


\section{CHAPTER 1}

\section{MICRORNA REGULATION AND MEDULLOBLASTOMA DEVELOPMENT}

\subsection{INTRODUCTION}

The most common pediatric malignancies are leukemia/lymphoma (40\%) and primary central nervous system (CNS) tumors (30\%). There are very few genetic changes identified in childhood malignancies e.g. fewer p53 mutations. These changes often arise in embryonal precursor cells where aberrant signaling in normal development (1). Despite advances in recent years that have resulted in an improvement in the survival rate of childhood cancers ( $80 \%$ ), these cancers still remain the most common cause of childhood death due to disease in western countries (2).

\subsection{CHILDHOOD BRAIN TUMORS}

Collectively, tumors of the brain are the most common solid tumors and most chemoresistant neoplasms of childhood and adolescence. Malignant tumors of the brain account for $21 \%$ of all cancers and $24 \%$ of all cancer-related deaths in this age group. They account for 4.3 cases per 100,000 people yearly in the U.S. and are among the leading causes of cancer-related morbidity and mortality during childhood $(3,4)$. CNS tumors show diverse histologies, location and survival. Treatment regiments for brain tumors involve surgery, radiation and chemotherapy. Complications as a result of treatment are of significant concern, as long term side effects are common in surviving patients (5). Tumors of the CNS are classified based on the World Health Organization (WHO) 
classification system of biological behavior of tumors, which includes a grading scale corresponding to aggressiveness (6). The malignancy scale is crucial for determining adequate therapies. In children these grades are from Grade I to Grade IV, where Grade I applies to lesions that have low proliferative potential and that are often correlated with good prognosis. Grade IV applies to high-proliferative, mitotically active cells and is often associated with fatal outcomes (6). A further sub-division exists whereby tumors are grouped based on the site of origin and type of cells. The most common childhood CNS tumors are located in the posterior fossa: astrocytoma (44\%), medulloblastoma (19\%) and ependynoma (10\%) (7).

\subsubsection{Treatment}

Standard therapy for brain tumors consists of surgery followed by radiation to the entire craniospinal axis with boosts to both the primary tumor site and focal CNS metastatic sites. Following diagnosis, location and histology (type and grade) of the tumor determine if the tumor is surgically accessible. Improved techniques such as functional imaging and diffusion tensor imaging help the surgeon map the brain and define tracts and connections, leading to better tumor resection management and less harm to normal tissues (8). Low grade glioma is completely resected and no further treatment is needed. Medulloblastoma and ependymoma, on the other hand, are often only partially resected through surgery. In such cases radiation and chemotherapy are necessary after surgery (9).

The most common radiation technique is craniospinal radiotherapy (CSRT). Patients treated with CSRT receive radiation to the craniospinal axis with an additional boost to 
the tumor. Novel radiation techniques are available and are in clinical trials including conformal radiation therapy (CRT) that incorporates three-dimensional CT and MRI imaging, allowing more precise planning and delivery, which helps to identify the tumor and surrounding critical structures.

A variety of chemotherapeutic agents are effective against CNS tumors, including platinum compounds, nitrosureas, cyclophosphamide, iphosphamide, temozolomide, etoposide and vincristine. Children who have undergone both chemotherapy and radiation therapy have a significantly higher survival rate. Chemotherapy eligibility depends on the type and stage of tumor, and age of the patient. In children younger than 3years with unresectable tumors, chemotherapy is used to delay radiation therapy or, at best, reduce the dose while trying to maintain high cure rates. Combinatorial chemotherapy is considered most effective when used with surgery and radiation treatment to control local and disseminated disease (9).

\subsubsection{Late Effects}

Late effects are likely the result of the complex interactions between the tumor, different treatment modalities and individual characteristics in a growing and developing child (7). Patients receiving radiation therapy are at risk of developing side effects that negatively affect cognitive, endocrine and neurological functions (5). Radiation has been reported to cause neuro-endocrine abnormalities, personality changes and neuro-cognitive sequelae for surviving children (5). Reduced IQ has been reported for children receiving whole brain radiation $(10,11)$; while endocrine sequelae depend on dose of radiation and age of 
the patient. Patients receiving as low as 16 gray $(\mathrm{Gy})$ acquire damage to the hypothalamic-pituitary axis with abnormalities in production of growth hormones (12). Patients receiving higher doses, $>40 \mathrm{gy}$, develop endocrine and reproductive dysfunction (e.g. decreased production of thyroid and follicle stimulating hormones, adrenocorticotrophic hormone, and luteinizing hormone) (12).

\subsection{MEDULLOBLASTOMA}

Medulloblastoma is the second most frequent brain tumor in children after pilocytic astrocytoma. Most medulloblastomas occur in the first decade of life $(13,14)$. Medulloblastoma is an embryonal tumor of the brain, analogous to Wilms tumor of the kidney and adrenal neuroblastoma (15). Its embryonal nature is underlined by its high incidence in infants and children and by its undifferentiated, immature appearance, which resembles developing neural tissue (15). It is a densely cellular, midline cerebellar tumor that arises over the roof of the fourth ventricle (15). Despite debates over its origin and nomenclature, medulloblastoma is grouped under the name primitive neuroectodermal tumors (PNET) because it was thought historically that all embryonic tumors originated from a common precursor cell of the subependymal matrix in the CNS (15). Gene profiling technologies have demonstrated that embryonic brain tumors are a heterogeneous group of neoplasms and that these tumors should be classified based on tumor location, histology and patterns of differentiation (16). Increasing evidence suggest that medulloblastoma may originate from precursor cells (e.g. granule cells) in the external granular layer (EGL) of the cerebellum $(16,17)$. The development of medulloblastoma follows a specific sequence of events [(18), Fig. 1]. The less aggressive 
classic and desmoplastic medulloblastoma subtypes generally arise upon constitutive activation of wingless (Wnt) or sonic hedgehog (Shh) signaling. Subsequent activation of MYC, through gene amplification or over-expression, coupled with chromosome 17p loss gives rise to the more aggressive, metastatic large cell/anaplastic medulloblastoma (Fig.1). Most medulloblastoma appear sporadically except for a small fraction of patients that harbor germ-line mutations in tumor suppressor genes, such as those in Gorlin syndrome (19) and Turcot syndrome (20).

\subsubsection{Histopathology}

Based on histopathology, medulloblastoma are divided in four subtypes: classical medulloblastoma, desmoplastic/nodular medulloblastoma, medulloblastoma with extensive nodularity (MBEN) and large/anaplastic medulloblastoma [LA/C, $(6,21)]$. Two tissue patterns are also identified: medulloblastoma with myogenic differentiation (medullomyoblastoma) and medulloblastoma with melanotic differentiation (melanocytic medulloblastoma) (6).

Classic medulloblastoma accounts for $65 \%$ of cases and is composed of diffused masses of small, undifferentiated oval or round cells. Desmoplastic medulloblastoma account for $25 \%$ of cases and comprise of firm and consistent stroma made-up of recticulin-free nodule surrounded by proliferative cells. MBEN accounts for $5 \%$ of cases and differs exhibits a markedly expanded lobular architecture and advanced neuronal differentiation. The LA/C subtype accounts for $5 \%$ of cases and are characterized by spherical cells that have large nuclei, open chromatin and prominent nuclei. 
External granule Layer cells

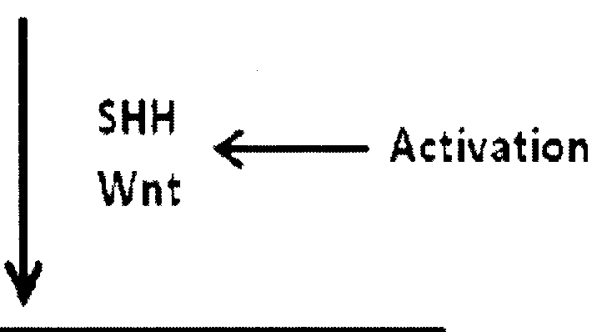

-Classic medulloblastoma

- Nodular/desmoplastic medulloblastoma

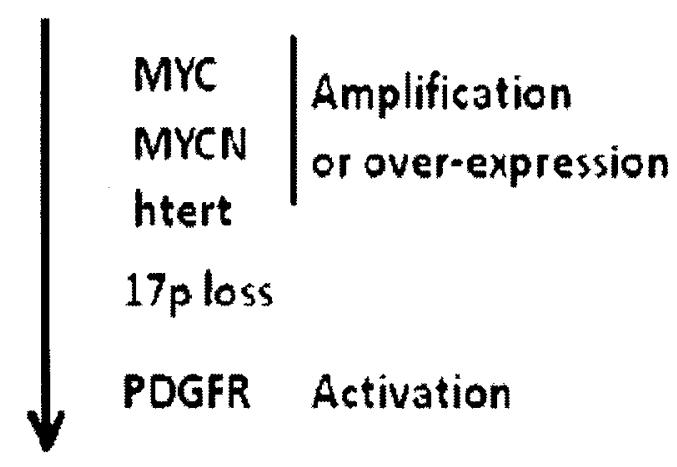

Large cell/anaplastic medulloblastoma

Fig. 1: Schematic representation of the molecular progression of medulloblastoma 


\subsection{MEDULLOBLASTOMA ETIOLOGY}

\subsubsection{A Change in Normal Development?}

It is accepted that there is a link between embryonic development and the biology of embryonic tumors (22). Many genes that were initially identified as oncogenes and tumor suppressor genes have been identified as the key regulators of normal developmental events. This is particularly true in embryonic tumors of the CNS (22). The majority of these genes regulate the components of Wnt and Shh signaling pathways which activate c-Myc. It is also clear that many of the pathways required for cerebellum development are activated in medulloblastoma $(16,17)$.

\subsubsection{Wnt Signaling}

The Wnt signaling pathway (Fig. 2) is a critical regulator of stem cell (SC) self renewal and is essential for proper embryonic development. It tightly controls intercellular communication in multiple developmental events and has been implicated in several diseases, particularly cancer $(23,24)$. The Wnt cascade regulates cell proliferation, cell fate specifications and cell differentiation through its major effector $\beta$-catenin (23). The stability of $\beta$-catenin is regulated by the destruction complex that induces adenomatous polyposis coli (APC), Axin, glycogen synthase kinase- $3 \beta$ (GSK-3 $\beta$ ) and casein kinase $1 \alpha$ (CK1 $\alpha$ ). In the absence of the Wnt ligand, the two scaffolding proteins APC and Axin bind newly synthesized $\beta$-catenin. GSK-3 $\beta$ and CK1 $\alpha$ then phosphorylate a set of conserved Ser and Thr residues on $\beta$-catenin. This results in the ubiquitination and degradation of $\beta$-catenin by the proteosome $(23,25)$. Upon activation of the Wnt ligand, the family of seven transmembrane Frizzle (Fz) receptors is engaged with the low-density 
lipoprotein (LDL) receptor related protein (LRP) complex, Lrp5/6. Binding of Wnt to the FZ-LRP facilitates reconfiguration of the FZ transmembrane domain. The intracellular proteins Dishevelled (Dsh) and Axin are recruited to the cell membrane, and phosphorylation of the cytoplasmic compartment of LRP occurs. In addition, relocation of Axin to the membrane leads to inhibition of $\beta$-catenin phosphorylation and subsequent inactivation of the destructive complex. This elevates cytoplasmic $\beta$-catenin protein levels, causing $\beta$-catenin to enter the cell nucleus, where it interacts with members of the $\mathrm{T}$ cell factor/lymphoid enhancer factor (Tcf/Lef) family of transcription factors. In the absence of Wnt signaling, Tcf/Lef interacts with Groucho and histone deacetylases such as Rpd3 to act as repressor of Wnt target genes (26). The binding of $\beta$-catenin to Tcf/Lef relieves the repressive activity of Groucho, and thus activates $\mathrm{Tcf}$ target genes such as cellular myelocytomatosis virus related oncogene (c-Myc) and cyclin D1 [(23, 25), Fig. 2]. Activation of Wnt signaling pathway is found in up to $25 \%$ of sporadic medulloblastoma (27). For instance, mutations in components of the Wnt pathway, such as CTNNBq (encoding $\beta$-catenin), APC and Axin, have been demonstrated in approximately $15 \%$ of sporadic medulloblastomas (28-30). Several studies using gene expression arrays have shown that Wnt signaling is activated in a distinct molecular subset of medulloblastomas $(16,31,32)$.

\subsubsection{Sonic Hedgehog (Shh) Signaling}

Shh signaling is known to regulate growth and patterning during embryonal development. Recent data suggest that Shh signaling is essential for SC maintenance and regeneration (33). Germ-line mutations that affect Shh signaling activity are associated with 
developmental disorder, whereas mutations activating the pathway are linked to multiple forms of cancer including medulloblastoma (34). In the absence of Shh, the 12-span transmembrane cell surface receptor Patched (Ptch) acts to inhibit the activity of the 7span transmembrane receptor-like protein Smoothened (Smo). Lack of Smo activity results in phosphorylation of Gli2/Gli3, which is further sequestered in the cytoplasm, where Gli2/Gli3 forms a complex with Fused and Costal2. Subsequently, Gli3 is recognized by $\beta-\operatorname{TrCP}$ and proteolytically processed to generate the truncated repressor form GliR. This truncated form of Gli3 represses a subset of Shh target genes (33). The binding of Shh to Patched releases inhibitory activity of Smo, which triggers the activity of Gli2 (GliA) by inhibiting the major intracellular inhibitor of Gli, Suppressor of fused. Free GliA can translocate to the nucleus and activate the expression of target genes such as MYC, Cyclin D1, Gli-1and Patched [(33), Fig. 2]. Gorlin syndrome, in which patche (Ptch) is mutaed, is an autosomal dominant disorder that predisposes individuals to developmental defects and cancer. About 1-2\% of medulloblastoma are attributed to the syndrome (35). Abnormal Shh signaling activity occurs in approximately $20 \%$ of sporadic medulloblastomas $(31,32)$.

\subsubsection{Other Important Signaling Pathways in Medulloblastoma Etiology} c-MET (MET or MNNG HOS) is a proto-oncogene that encodes a protein known as hepatocyte growth factor receptor [(HGFR) (36)] the only known ligand for the MET receptor. MET is activated by it ligand HGF eventually leads to phosphorylation and activation of the p85 regulatory subunit of phosphatidylinositol-3 kinase (PI3K) or indirectly through the scaffolding protein Gab1(37). 


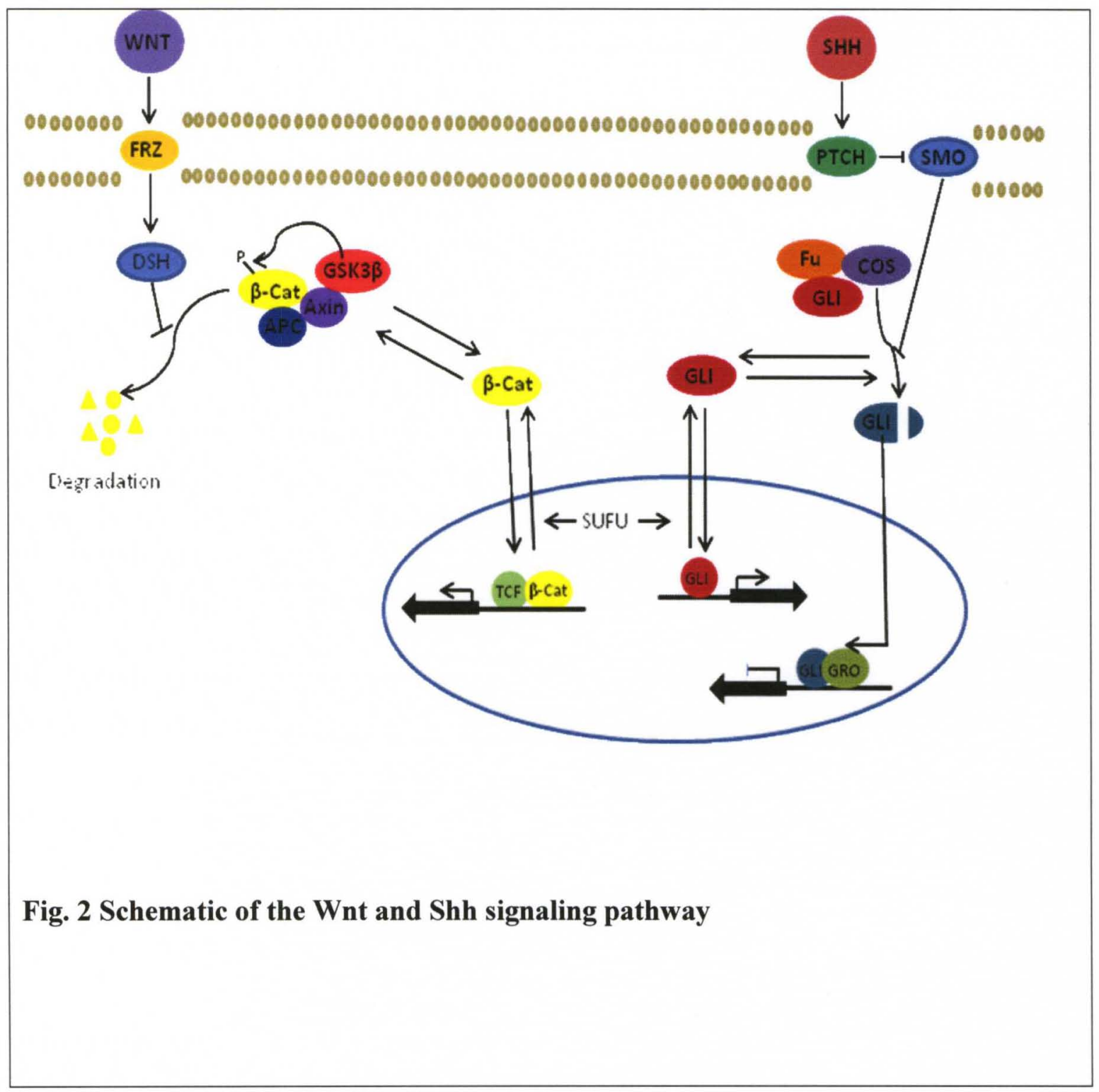


Aberrantly active MET triggers tumor growth, angiogenesis and metastasis, and this correlates with poor prognosis in cancer. MET regulates tumor growth, angiogenesis and metastasis, and this correlates with poor prognosis of cancers. MET is deregulated in many types of human malignancies, including medulloblastoma. HGF induces tumor cell proliferation, anchorage-independent growth, and cell cycle progression beyond the G1-S checkpoint (38). HGF also protects medulloblastoma cells against apoptosis induced by chemotherapy. This cytoprotective effect was associated with reduction of pro-apoptotic cleaved poly (ADP-ribose) polymerase and cleaved caspase-3 proteins and required PI3K activity (39). HGF induces $M Y C$ mRNA and c-Myc protein in established and primary medulloblastoma cells.

Myc has emerged as an important prognostic indicator and modulator of medulloblastoma malignancy. It is central to the activity of the Shh, Wnt, c-Met and insulin growth factor (IGF) pathways which are usually deregulated in medulloblastoma (31).

\subsubsection{Biological and Genetic Marker}

The neurotrophin-3 (NT-3) receptor TRKC was the first TRK used as a marker to predict clinical outcome, and now several TRKs have been correlated with survival in medulloblastoma patients. NT-3 activates TRKC and regulates proliferation, differentiation and cell death of the granule cells of the developing cerebellum. High TRKC mRNA expression is associated with a favorable outcome in medulloblastoma, with $5 y$ free survival rate of $89 \%$ vs $46 \%$ for low TRKC mRNA expression (40). 
Patients with deregulated Wnt signaling belong to a distinct molecular sub-group of medulloblastoma $(27,41)$. Medulloblastoma with Wnt activation are usually associated with chromosomal loss of $6 p(41)$. Patients with $\beta$-catenin necleopositivity have a better overall survival (OS) rate compared to patient without Wnt activation, $92 \%$ vs $65 \%$ (27, 41). Genetic abnormalities of Shh signaling, as seen in response to mutated Ptch1 (the Shh receptor which acts as a repressor in the absence of the ligand), are responsible for Gorlin syndrome, which exhibits high medulloblastoma incidence (42). Mutation of Shh negative regulators, such as Ptch1, SuFu and Ren/KCTD11 have all been reported in sporadic medulloblastoma. Mutations in these upstream regulators have also been shown to regulates Shh target genes including MYC $(17,43)$. Genomic amplification of MYC and the neuroblastoma-derived MYCN is strongly correlated to poor prognosis [5 yr OS: amplified MYC $13 \%$ vs not amplified $72 \%(44,45)]$. Low expression of MYC mRNA indicates a good prognosis, particularly when found in combination with high TRKC expression (46).

The most characteristic chromosomal abnormalities in medulloblastoma are $17 \mathrm{p}$ deletion and 17q gain. Approximately $30 \%$ of medulloblastoma patients harbor at least one of these abnormalities (44). The 5yr OS for 17q gain (55\%) and isochromosome (35\%) are better predictors of outcome than $17 \mathrm{p}$ deletions [ $78 \%$ (44)].

\subsection{MYC ONCOGENE}

\subsubsection{Gene and Protein Product}


The $M Y C$ gene was originally identified in avian retroviruses as an oncogene responsible for inducing myelocytomatosis in birds (47). Its human homologue, c-Myc, was found to be evolutionarily conserved (48). In mammals, there are four related genes in the family, c-Myc, N-Myc, L-Myc and S-Myc, with all sharing similar structural framework. c-Myc is a proto-oncogene and is located on chromosome $8 \mathrm{q} 24.21$. The gene product is a nuclear phosphoprotein with a short half-life of 20-30 mins that is subsequently ubiquitinated for proteosome degradation (49).

\subsection{2. c-Myc as a Transcription Factor}

$M Y C$ is a basic Helix-Loop-Helix leucine zipper (bHLHZip) protein that heterodimerize with Max, another bHLHZip protein, with binding ability to consensus 5'-CAC[C/T] GTG-3'DNA sequences called E-boxes. The basic region promotes sequence-specific DNA binding; the HLHZip domain confers protein-protein interaction while the Zip domain functions in maintaining dimerization. It is believed that Myc exerts its main functions through gene regulation by recruiting transcriptional cofactors involved in modulation of RNA polymerase II function and chromatin structure, including histone acetyl transferase (HAT) and transformation/transcription domain-associated protein (TRRAP) recruitment. These are engaged through interactions with the conserved Mycbox 2 (MB2) in the transactivation domain (TAD) or with the bHLHZip domain (50-52). While the MB2 domain (spanning residues 128-143) is required for cell transformation $(49,53)$, the MB1 domain (residues 47-62) is required for c-Myc activity and degradation $(51,54)$. MB3 plays a role in cellular transformation and MB4 in Myc-induced focus 
formation (55). All four MBs are found in both c-Myc and N-Myc, whereas L-Myc lacks MB3 (56).

\subsubsection{Downstream Effects of c-Myc}

MYC protein product is a transcription factor that regulates the expression of $\sim 15 \%$ of all genes in the cell. Due to the plethora of target genes c-Myc regulates, it is viewed as a multifunctional protein with the ability to regulate activities such as cell cycle, growth and metabolism, differentiation, apoptosis, transformation, genomic instability and angiogenesis $(50,51)$.

\subsubsection{Cell Cycle and Differentiation}

As Myc is essential for G0/G1 to S-phase cell cycle progression, cells with activated Myc have a shortened G1 phase $(57,58)$. Myc abrogates the transcription of cell cycle checkpoint genes (for example, Gadd45 $\alpha$ and Gadd153) and inhibits the function of cyclin-dependent kinase (CDK) inhibitors, either through directly repressing gene transcription or indirectly through degradation or sequestration. Myc also promotes cell cycle progression by activation of cyclin D1, cyclin D2, and cyclin A, as well as Cdk4, cell division cycle 25A (CDC25A), and E2 promoter binding factor (E2F) $1 / 2(59,60)$. Some examples of c-Myc target genes are ornithine decarboxylase (ODC), and Cyclin E $(50,61)$. Ornithine decarboxylase (ODC) controls polyamine biosynthesis which is essential as s modifier of ion transport channels, regulate protein translation, Okazaki fragment joining during DNA replication and progression through $\mathrm{S}$ phase of cell cyclin (62). Cyclin E, with its partner kinase Cdk2, regulates the G1 to S-phase transition and 
promotes DNA replication (63). c-Myc mediated ODC and Cyclin E up-regulation may contribute to an oncogenic phenotype as ODC over-expression in mouse fibroblasts results in transformation (64).

MYC has also been found to be important during cellular differentiation. Downregulation of Myc is required for cells to exit the cell cycle and undergo differentiation. This important point of regulation is further enforced by the induction and function of the Mxd family members in response to differentiation cues $(57,65)$.

\subsubsection{Cell Growth/Proliferation}

The ability of Myc to promote cell growth has been demonstrated in vitro and in vivo. Myc enables cell growth by providing the cell with an abundant supply of several classes of basic building blocks as well as increasing cell metabolism and protein synthesis (66). Myc-induced cell proliferation is potentiated by several Myc target genes, including those associated with cellular metabolism, ribosomal and mitochondrial biogenesis, and protein and nucleic acid synthesis $(67,68)$. Gadd $45 \alpha$ and ODC are specific c-Myc targets that have been implicated in c-Myc-mediated cell growth and proliferation. Equally, c-Myc has been shown to down-regulate both basal and stress-induced Gadd45 expression (69). Gadd45 $\alpha$ has been shown to function in growth arrest and in apoptosis following genotoxic stress (70).

\subsubsection{Genomic Instability}


S. Mai and colleagues showed that specific gene amplification occurs at a high frequency in cells with deregulated Myc (71). Additional research shows that Myc can promote chromosomal instability $(72,73)$. However, the role of Myc in genomic instability has been a subject of debate and controversy (74). Several mechanisms have been proposed, including increased levels of reactive oxygen species, alterations in chromosome structure (75), overcoming the p53 checkpoint (75), and induction of a DNA damage response and/or replication (76). Losing the high-fidelity organization of the genome is a hallmark of tumorigenesis that can clearly be associated with Myc deregulation.

\subsubsection{Metastasis and Angiogenesis}

Down-regulation of thrombospondin is vital to angiogenesis and is potentially achieved through Myc induction of the miR-17 92 cluster (77). The role of Myc expression during normal development is associated with proliferative expansion and cellular migration. Recent reports have demonstrated that c-Myc expression is required for the activation and expression of miR-9 (78-80). miR-9 negatively regulates E-cadherin, which results in activation of $\beta$-catenin and in turn contributes to cell metastasis and angiogenesis (80). The relevance to tumorigenesis is intuitive, with deregulated MYC preventing differentiation and promoting migration, leading to the features of aggressive, less differentiated, metastatic cancers.

\subsubsection{Induction of Apoptosis}

One of the functions of Myc is induction of apoptosis in response to cellular stress. Cyclin A and ODC are central to c-Myc-mediated apoptosis. Inhibition of ODC blocks 
inhibits apoptosis in c-Myc-over expressing cells, and forced expression of Cyclin A is sufficient to induce apoptosis under low serum conditions (81). c-Myc-mediated apoptosis correlates with regulation of the Fas receptor and its ligand as well as proapoptotic Bax (82). Despite modest change in Bax levels upon c-Myc overexpression (83), Bax appears to be essential for c-Myc-mediated apoptosis by indirect upregulation of upstream molecules like Caspase 8 (84). Furthermore, in response to c-Myc activation and MYC/Ras-induced transformation, p53 can be up-regulated and stabilized to induce cell cycle arrest or apoptosis (85). Furthermore, c-Myc-mediated apoptosis might be due to accumulation of reactive oxygen species (ROS) as a consequence of NF$\kappa \mathrm{B}$ inhibition (86).

\subsubsection{Regulation of 'Stemness'}

Experiments in transgenic mice have revealed that expression of c-Myc is essential for normal developmental control of haematopoietic stem cells (HSC) and neural stem cells $(87,88)$. Knockout of $M Y C$ in immature HSCs results in pancytopenia and rapid lethality (89). Likewise, c-Myc is crucial for self-renewal and maintenance of pluripotency in murine ES cells (90). More evidence demonstrating the importance of c-Myc in 'stemness' comes from experiments where murine and human somatic cells were reprogrammed to pluripotency through the generation of induced pluripotent stem cells (iPS) by retrovirus-mediated introduction of Oct3/4, Sox2, Klf4 and c-Myc (91). Although a recent report shows that c-Myc may be redundant in the conversion of somatic cells to iPS cells (92), there is still an implication for a novel 'stemness' function of c-Myc that may be important for controlling tumor-initiating cells. The pluripotent 
ability of c-Myc has been connected to its ability to positively regulate the cell cycle machinery (93). Consistently, down-regulation of c-Myc correlated with the differentiation of pluripotent cells (90).

\subsection{4. $M Y C$ in Medulloblastoma}

Over-expression of c-Myc in medulloblastoma has been associated with poor prognosis and the prognostically dismal large cell/anaplastic medulloblastoma subtype (94-96). Shh signaling corporate with c-Myc to enhance tumorigenicity of Nestin-expressing neural progenitors that are present in the cerebellum at birth. The Nestin-expressing neural progenitor cells can act as the cells-of-origin for medulloblastoma (97). c-Met induces cMyc, which mediates medulloblastoma cell proliferation, cell cycle progression, apoptosis and increased cell size (39). In cultured granule neuron precursor (GNP) cells, insulin growth factor (IGF) signaling stabilizes Myc by inhibiting GSK3 $\beta$-dependent phosphorylation, thus preventing degradation of Myc. Similarly, Myc can substitute for IGF signaling in a mouse model of Shh-induced medulloblastoma (98). Therefore, Myc appears to play a central role in mediating the effects of the signaling pathways in medulloblastoma including Shh, Wnt, c-Met, and IGFR. Therefore, it represents an attractive target for the therapy of this neoplasm, especially the very aggressive large cell/anaplastic subtype. Unfortunately, clinically useful inhibitors of Myc are not available to date.

Over-expression of c-Myc is found in $~ 31-64 \%$ of medulloblastoma patients (96), but only $5-15 \%$ of them have $M Y C$ gene amplification $(94,99,100)$. Various means of 
amplification-independent c-Myc activation have been identified in other human cancers: chromosomal translocations in Burkitt's lymphoma constitutively activate mRNA expression (101); c-Myc expression in multiple myeloma are increased 20 -fold by a mutation in the $5^{\prime}$ untranslated internal ribosome entry segment (102); mutations at the Myc intron factor binding site in mucosa-associated lymphoid tissue lymphomas removes a negative regulatory sequence (103); and APC or $\beta$-catenin mutations in colon cancer activate the c-Myc promoter at a $\beta$-catenin/Tcf-4 transcription factor binding site (104). APC and $\beta$-catenin mutations have also been found in a small fraction of medulloblastoma (30), and nuclear $\beta$-catenin staining was reported in $18 \%$ of medulloblastoma studied (29). Therefore, genomic amplification-independent activation of c-Myc is a likely mechanism for tumor progression of medulloblastoma.

\subsubsection{Regulation of $M Y C$}

In normal cells Myc expression and activity is regulated at multiple levels. At the transcriptional level, Myc is regulated by signal transduction pathways that are activated both during normal development and in cancer. The most important include Shh, Wnt, Notch, RTK signaling and transforming growth factor (TGF)- $\beta$. Posttranslationally, cMyc is regulated through sequential and reversible phosphorylation at two conserved sites, threonine 58 (T58) and serine 62 (S62), located on the amino-terminal TAD of the protein (54). T58 phosphorylation stimulates ubiquitination and proteasomal degradation by $\mathrm{SCF}^{\mathrm{FBW} 7}$ (105), while $\mathrm{S} 62$ phosphorylation stabilizes c-Myc. It has been hypothesized that GSK-3ß-mediated phosphorylation of c-Myc at T58 and dephosphorylation at S62 allows for binding of ubiquitin ligase $\mathrm{FBW} 7$ and recruitment of $\mathrm{SCF}^{\mathrm{FBW} 7}$ complex to 
direct c-Myc degradation. Coupled to FBW, F-box protein Skp2 and Hect H9 are two other ligases involved in c-Myc turnover (106). Skp2 effects transcriptional activation and degradation (107) while Hect $\mathrm{H} 9$ promotes transcriptional activity only (106). Proteins such as Ras, frequently altered in human cancers, can lead to inhibition of GSK$3 \beta$ activity and subsequent stabilization of c-Myc protein through loss of T58 phosphorylation (108). GSK-3 $\beta$ acts as a key protein in the canonical Wnt $/ \beta$-catenin signaling pathway by regulating the activity and nuclear transportation of $\beta$-catenin, which subsequently leads to transactivation of MYC (104).

\subsection{MicroRNA (miRNA)}

MiRNAs are small non-coding RNAs (18-25 nucleotides), involved in repression of gene expression by binding to the mRNA $3^{\prime}$ 'untranslated region ( $\left.3^{\prime} U T R\right)$, and effecting either translational repression, or mRNA degradation in animals, plants and protozoa. In mammals miRNAs are predicted to control the expression of more than $60 \%$ of all protein-coding genes (109), and are thereby involved in nearly all cellular processes.

\subsection{1. miRNA Biogenesis}

Individual miRNAs can be transcribed separately from a single transcriptional unit, which can encode a single miRNA or a cluster of distinct miRNAs; or they can be processed directly from other RNA species, such as introns (Fig. 3). Transcription of miRNAs by RNA polymerase II results in primary miRNA transcripts (pri-miRNAs), which are 5' capped and polyadenylated at the 3' end. The pri-miRNAs transcript is then cleaved in the nucleus by Drosha, an RNase III endonuclease, in association with 
DGCR8/Pasha, the double-stranded RNA-binding domain protein, in a complex known as the microprocessor complex (110). Drosha cleaves both strands of the stem at sites near the base of the primary stem-loop to generate an intermediate called precursor miRNA (pre-miRNA). The pre-miRNA is exported out of the nucleus with the aid of the nuclear membrane bound exportin 5. In the cytosol the RNase III domain-containing nuclease, Dicer, cleaves the terminal loop to generate the $\sim 22$ nucleotide mature miRNA. Dicer functions in combination with the double-stranded RNA-binding domain protein TRBP and PACT (111). The mature double-stranded RNA duplex that results from Dicer cleavage is unwound and then loaded into the RNA-induced silencing complex (RISC). RISC is a complex composed of Dicer, TRBP and a protein of the argonaute superfamily (112). The complex identifies target mRNA based on sequence complementarity between the miRNA and the associated single-stranded mRNA's 3'UTR, resulting in either mRNA cleavage or translational repression (113). Two basic mechanism have been proposed for miRNA gene silencing: initiation block, where the miRISC inhibits translation initiation by interfering with the eIF4F-cap recognition and $40 \mathrm{~S}$ small ribosomal subunit recruitment or by antagonizing $60 \mathrm{~S}$ subunit joining and preventing $80 \mathrm{~S}$ ribosomal complex formation (114); and post-initiation block, where the miRISC inhibit translation by inhibiting ribosome elongation, inducing ribosome drop-off, or facilitating proteolysis of nascent polypeptide (115).

\subsection{2. miRNAs and Cancer}

About $50 \%$ of annotated human miRNAs are mapped in fragile regions of chromosomes, which are areas of the genome prone to 'genomic earthquakes, that can trigger 
chromosome rearrangement, disrupt genes and alter gene regulation. These genomic regions are associated with various human cancers (116-118). The relevance of miRNAs to cancer is highlighted by their differential expression patterns in cancerous cells compared to normal cells. Several miRNAs have emerged as candidate components of oncogene and tumor-suppressor networks. The miR-17 92 cluster $(119,120)$, and miR155/BIC (121) have been implicated as proto-oncogenes in B-cell lymphomas. On the other hand, let-7 tumor suppressive function has been validated in various cancers $(122$, 123). Many of the oncogenic or tumor-suppressor miRNAs target cell cycle regulators, and this interaction may at least partially explain the oncogenic function of the miRNA alteration in cancer cells. miRNAs act as oncogenes and tumor suppressor genes and induce all of the six hallmarks of malignant cells.

\subsubsection{Self-sufficiency in Growth Signals}

To become independent from external growth factor signals and evade tissue homeostasis, tumor cells activate different pathways to sustain cell proliferation and survival such as the RAS proteins, H-RAS, K-RAS, and N-RAS. RAS oncogenic signaling is activated in response to decreased levels of let-7, a well-documented posttranscriptional RAS regulator that is inversely correlated with RAS expression in solid and hematologic malignancies (122). Let-7 down-regulation is associated with poor prognosis in lung cancer patients (124) and head and neck squamous cell carcinoma patients (125). Let-7 acts as a tumor suppressor by targeting oncogenes such as RAS and HMGA2 and is down-regulated in many solid cancers (126). 
Reduced Let-7expression result in up-regulation of the high-mobility group AT-hook 2 (HMGA2), which helps tumor cells grow in an anchorage-independent manner and not undergo apoptosis after they lose contact with the basal membrane. The clinical relevance of let-7's anti-proliferative effects was recently demonstrated by let-7-induced tumor regression in in vivo murine lung cancer models (124). Also, miR-21, one of the most frequently up-regulated miRNAs in solid tumors, also participates in RAS oncogenic signaling. AP1 that acts downstream of RAS transcriptionally induce miR-21which exerts its oncogenic effect by keeping phosphatase and tensin homolog (Pten) and PDCD4 in check (127). PDCD4, in turn negatively controls AP1, thus AP1-induced miR21 represents a positive feedback loop that sustains AP1 activity in response to RAS (127). miR-21 overexpression enhances tumorigenesis, and genetic deletion of this oncogenic miRNA partially protects against tumor formation in transgenic mice (128, 129).

\subsubsection{Insensitivity to Anti-Growth Signals}

The retinoblastoma (RB) pathway is one of the major cell cycle regulatory routes that is altered in almost all human cancer cells (130). RB is a transcriptional repressor that inhibits members the E2F family, resulting in proliferative arrest and inhibition of the expression of genes required for cell cycle progression such as MYC. The RB pathway is defective in a wide range of human tumors, resulting in deregulated and hyperactive E2F in transformed cells and uncontrolled cell proliferation (131). E2F transcription factor activities are controlled at the post-transcriptional level by a series of miRNAs including miR-17-5p, miR-20a, miR-106b, and miR-92 (119, 132, 133). Furthermore, E2F- 
activating transcription factors have been shown to regulate the expression of miRNAs $(132,133)$. Unbound E2F increases miR-17 92 and miR-106b 25 expression which in turn keeps E2F levels in check through miR-17-5p and miR-20a (119), thereby participating in the delicate equilibrium between cell proliferation and apoptosis. Upregulated miRNAs from these clusters target apoptotic and growth inhibitory proteins such as BIM and p21 (132). Under physiologic conditions, the miR-17 92 cluster limits MYC activation by dampening the E2F positive feedback loop. In tumors with MYC activation, the miR-17 92 cluster protects cells from Myc-induced apoptotic E2F response, leading to uncontrolled cellular proliferation. Also, miR-106b 25 family members are found to be induced in gastric cancer (132) and negatively regulate excessively high E2F1 expression levels that cause tumor cell apoptosis. miRNAs also play roles in activating cell cycle progression by targeting cell cycle checkpoint proteins. The miR-15a 16-1 cluster induces cell cycle arrest at G1 phase by targeting critical cell cycle regulators such as CDK1, CDK2 and CDK6 as well as cyclins (D1, D3 and E1) $(134,135)$. In fact, these major cell cycle kinase complexes are regulated by several other miRNAs. CDK4 or CDK6 mRNAs are targeted by miR-24, miR-34a, miR-124, miR125b, miR-129, miR-137, miR-195, miR-449 and let-7 family members $(136,137)$. As expected, most of these miRNAs display anti-proliferative properties, function as tumor suppressors and are inactivated in cancer by different mechanisms (138). Finally, some cell cycle inhibitors of the INK4 or Cip/Kip families are also tightly regulated by miRNAs. p16 ${ }^{\mathrm{INK} 4 \mathrm{a}}$, a CDK4/6 specific inhibitor, is controlled by miR-24 and miR-31 (139). Both miRNAs are involved in the regulation of cell proliferation and progress 
through the cell cycle at least in part by regulating the levels of the p16 ${ }^{\mathrm{INK} 4 \mathrm{a}} \mathrm{CDK}$ inhibitor.

\subsubsection{Apoptosis}

Apoptosis is a physiologic self-destruction mechanism that leads to the removal of unwanted cells. P53 is the most widely studied transcription factor; it controls the expression of several genes to promote tumor suppression. p53 responds to diverse cellular stresses and regulates target genes involved in DNA integrity, cell cycle arrest at the G1/S checkpoint, and apoptosis. It is the most commonly mutated gene in human cancer. miRNAs such as miR-34, miR-192/215, miR-107, and miR-145 are known transcriptional targets of p53 (140). The cancer-associated genomic region $1 \mathrm{p} 36$, which is lost or rearranged in many tumor types, including those from neural, epithelial, and hematopoietic tissues, contains the candidate tumor suppressor miR-34a. The miR-34 family is composed of three evolutionarily conserved members: miR-34a, miR-34b, and miR-34c. miR-34a functions as a potent suppressor of cell proliferation by modulating E2F signals (141) and induces p53 in a cell-type specific manner to activate apoptosis. Other major pro-apoptotic miRNAs with reduced expression in tumors include the miR15a/16-1 cluster, which represses anti-apoptotic $\mathrm{Bcl} 2$ protein and activates the intrinsic apoptotic program APAF-1-caspase-9-poly (ADP-ribose) polymerase (142). miR-15a and miR-16-1 are clustered on human chromosome 13q14, which is frequently lost or downregulated in B-cell chronic leukemia and several solid tumor types (142).

\subsubsection{Limitless Replicative Potential}


Cellular senescence is a physiologic withdrawal from the cell cycle in response to a multitude of different stress stimuli, including oncogene activation, and telomere deregulation (143). It is executed by two important cell cycle inhibitors, p21 and p16 (144) which are activated upon cellular stress. Also, various stresses can induce senescence, but p53 and RB have been identified as critical pathways common to the initiation, execution, and maintenance of senescence-associated growth arrest. P53activated miRNAs such as miR-34 are also important in senescence by modulating the E2F signaling pathway $(145,146)$. Furthermore, 15 miRNAs have been found to be down-regulated in senescent cells and breast cancers harboring wild-type p53, which demonstrated that these miRNAs are repressed by p53 in an E2F1-mediated manner (147). Recently, the protein subunit TERT of telomerase was found to be a direct target of regulation by miRNAs such as miR-138, miR-143, and miR-146a $(148,149)$.

\subsubsection{Angiogenesis}

Tumor cells turn on an "angiogenic switch" to produce large amounts of pro-angiogenic factors and promote vascularization. Hypoxia adaptation is an essential cellular response that is controlled by vascular endothelial growth factor (VEGF), which is also regulated by the oxygen-sensitive master transcription factor hypoxia-inducible factor 1 (HIF-1). HIF-1 expression is controlled by specific miRNAs such as the p53-induced miR-107 and miR-92 that target the Von Hippel-Lindau gene whose product is responsible for HIF-1 degradtation $(150,151)$; it, in turn, controls the expression of other miRNAs (152-154) that fine-tune adaptation to low oxygen tension. In tumor progression, hypoxia has been found to contribute to the modulation of miRNA expression, partly by direct HIF-1 
transcriptional activation of specific miRNAs such as miR-424 and miR-210 (152-154). These miRNAs have dual functions: they participate in the angiogenic process while helping the cell engage anti-apoptotic programs that sustain survival. miR-126 expression was found to be enriched in endothelial cells during angiogenesis and to repress negative regulators of the VEGF pathway (SPRED1 and PIK3R2) (155). miRNAs that are downregulated in hypoxic conditions, such as miR-16, miR-15b, miR-20a, and miR-20b, directly modulate VEGF expression levels. This creates a positive feed-forward loop in which hypoxia-repressed miRNAs reinforce the expression levels of a potent proangiogenic hypoxia-induced growth factor, such as VEGF (156). Lastly, individual miRNAs in the miR-17 92 cluster have effects on angiogenesis with miR-17-5p, miR$19 \mathrm{a}$ and miR-18a contributing directly to angiogenesis (157).

\subsubsection{Invasion and Metastasis}

The metastatic process involves multiple steps which culminate in tumor cells spreading to distant organs. The deregulation of epithelial-to-mesenchymal transition gives cells migratory and invasive properties that promote the dissemination of tumor cells and metastasis. Recent studies have shown that members of the miR-200 family (miR-141, miR-429, miR-200a, miR-200b, and miR-200c) are essential regulators of differentiation and epithelial cell character through the targeting of the zinc finger E-box-binding homeobox (Zeb) family of transcription factors $(158,159)$. Zeb1 and Zeb2 are master regulators of the mesenchymal phenotype and repress the transcription of genes containing E-box elements in their promoters, including E-cadherin. Twist, a metastasispromoting transcription factor, was found to induce miR-10b expression, whereas 
HOXD10, a homeobox transcription factor that promotes or maintains a differentiated phenotype in epithelial cells, is found to be a miR-10b target that is expressed at low levels in metastatic tumors (160). miR-21 does not only control cell survival and proliferation; it is also a master regulator of the metastatic process by directly modeling the cell cytoskeleton via TPM1 suppression $(161,162)$ and by indirectly regulating the expression of the pro-metastatic UPAR (via Maspin and PDCD4 direct suppression) (161), matrix metalloproteinases (via RECK and TIMP3) (163), and Pten direct suppression (164).

\subsubsection{Chemotherapeutic Importance of miRNAs}

RNA-based gene therapy can be used to treat cancer in two ways: by using RNA molecules against the mRNA of genes involved in cancer pathogenesis; or by directly targeting the miRNAs that participate in pathogenesis. It is recently reported that therapeutic miRNA delivery suppresses tumorigenesis in a murine liver cancer model: hepatocellular carcinoma cells exhibit reduced expression of miR-26a and systemic administration of miR-26a in a mouse model of hepatocellular carcinoma using adenoassociated virus results in inhibition of cancer cell proliferation, induction of tumorspecific apoptosis, and dramatic protection from disease progression without toxicity (165). Antagomirs are therapeutic RNA molecules that were originally designed to inhibit miRNAs $(166,167)$. Antagomirs' anti-cancer activity was demonstrated in a neuroblastoma model in which cholesterol conjugated antagomir-17-5p delivery resulting in regression of neuroblastoma in $30 \%$ of cases (168). Another method of targeting miRNAs is through locked nucleic acid (LNA)-mediated miRNA silencing. Efficient 
miR-122 silencing was achieved with three doses of $10 \mathrm{mg} / \mathrm{kg}$ LNA-anti-miRNA. MiR122 is an abundant liver specific miRNA, implicated in fatty acid and cholesterol metabolism and hepatitis $\mathrm{C}$ replication leading. The LNA-miR-122 be exhibited a longlasting and reversible decrease in total plasma cholesterol and no evidence of associated toxicities or histopathologic changes in the liver (169). Despite the promising result, the use of miRNA therapy has not been successful in humans due to constrains such the large size of miRNAs or anti-miRNA thereby failing one of the Lipinski rule of five for druglikeliness. In addition the molecules are likely recognized as foreign and elicit immune responses. Furthermore, miRNAs are expected to have low stability in vivo since they can be degraded by endogenous RNases.

\subsection{4. miRNAs and Medulloblastoma}

Peirson and associates were the first to demonstrate that miRNA could regulate medulloblastoma development. They showed that miR-124, a brain enriched miRNA, was significantly down-regulated in medulloblastoma and augmentation of miR-124 reduced medulloblastoma cell growth by targeting CDK6 (136). Since then, roles have been attributed to miRNAs in modulation of the Shh signaling and Notch (170). For example, HES1, the principal Notch-responsive gene, has been shown to be negatively regulated by miR-199b-5p, resulting in reduce cell proliferation and migration of medulloblastoma cells (171). Moreover, other investigators have focused on miRNA profiling of medulloblastoma cell lines in various cellular states and have identified important miRNA signature patterns in medulloblastoma development $(172,173)$. These 
experiments have all highlighted the importance of miRNA in medulloblastoma development and their therapeutic potential.

\subsubsection{Myc-miRNA Regulatory Network}

The transcription factor, c-Myc, also controls the production of many non-coding RNAs, including tRNA, rRNA and miRNAs. These RNAs are likely to contribute to the complex biology and pathology that is associated with changes in c-Myc expression (174). MYC and miRNAs form complex network, with a number of miRNAs reported to target MYC and c-Myc regulating the expression some miRNAs. J.T. Mendell and coworkers reported the direct induction of the miR-17 92 cluster by c-Myc (119). This cluster of six miRNAs is an oncogene and cooperates with c-Myc to accelerate tumor development in a mouse model of B-cell lymphoma (120). This cluster, as well as the paralogous cluster miR-106a 92 induced by $\mathrm{N}-\mathrm{Myc}$, promotes cell cycle progression in neuroblastoma cells (175). Both c-Myc and MYCN also induce miR-9, a miRNA that targets $C D H 1$, encoding E-cadherin, leading to increased cell motility and invasiveness, as well as increased tumor angiogenesis (80). c-Myc participates in the tumor-associated repression of miRNAs by directly repressing several miRNA clusters such as miR-15a16-1, miR-22, miR-23a/b, miR-26, miR-29 and several let-7 clusters (176). Many of these miRNAs down-regulated by c-Myc are known tumor suppressors and target critical cell cycle regulators $(177,178)$. Although most of these miRNAs are down-regulated through the transcriptional repressor activity of c-Myc, c-Myc may also lead to miRNA down-regulation by alternative mechanisms. For example, c-Myc is able to block the maturation of specific miRNAs by inducing the RNA binding proteins Lin 28 and Lin $28 \mathrm{~b}$ 
$(176,179)$, which are known to act as negative regulators of let-7 maturation at multiple levels including Drosha and Dicer processing (180). Repression of multiple miRNAs by c-Myc is likely to be an important mechanism contributing to the reduced function of miRNAs in cancer cells (180).

\subsection{RESEARCH THEME}

Given the convergence of miRNAs and c-Myc, my dissertation studies focus on: (i) whether and which miRNAs target c-Myc; (ii) whether the repression of $M Y C$ by miR$33 \mathrm{~b}$ is sufficient to reduce medulloblastoma tumor growth; (iii) whether we can identify the US Food and Drug Administration (FDA)-approved drugs that induce miR-33b expression to inhibit c-Myc expression and function in medulloblastoma; and, (iv) whether epigenetic silencing of miR-33b gene is responsible for the reduced sensitivity to lovastatin in HeLa cells.

First, I identified that miR-33b is a primate-specific negative regulator of c-Myc. The human miR-33b gene is located at $17 \mathrm{p} 11.2$, a genomic locus frequently lost in medulloblastomas. Through a small-scale screening with drugs approved by the FDA, I found that lovastatin upregulated miR-33b expression, reduced cell proliferation, and impaired c-Myc expression and function in miR-33b-positive medulloblastoma cells. In addition, a low dose of lovastatin treatment at a level comparable to approved human oral use reduced tumor growth in mice orthotopically xenografted with cells carrying miR$33 \mathrm{~b}$, but not with cells lacking miR-33b. This work presents a highly promising therapeutic option, using drug repurposing and a miRNA as a biomarker, against cancers 
that over-express c-Myc. I also revealed that the promoter of the $m i R-33 b$ gene is methylated in HeLa cells but not in medulloblastoma cells, which may contribute to the lower sensitivity to lovastatin of HeLa cells. This information is critical to future drug development against cancers other than medulloblastoma using miR-33b and c-Myc. 


\section{CHAPTER 2}

\section{MICRORNA-33B (MIR-33B) TARGETS MYCAND NEGATIVELY AFFECTS MEDULLOBLASTOMA GROWTH.}

\subsection{INTRODUCTION}

The $M Y C$ gene codes for the c-Myc onco-protein and is over-expressed in the majority of human cancers. It is now well established that the deregulated expression of c-Myc plays a significant role in human cancer development (181-183). The importance of c-Myc in carcinogenesis is supported in animal models; over-expression of $M y c$ in mouse liver induces hepato-cellular carcinoma, which regresses if Myc expression is inactivated (184). c-Myc is a transcription factor that exert its biological functions through activating and repressing target genes $(185,186)$. Its expression is tightly control and activated mitogenic cues resulting in cell proliferation (183).

Treatment of medulloblastoma remains problematic due to high doses of radiotherapy and chemotherapeutics that lead long-term toxicity. A major focus is the development of innovative approaches such as targeted therapies to reduce long-term toxicity. c-Myc is over-expressed in up to $\sim 31-64 \%$ of medulloblastoma (187) and has been associated with the large-cell/anaplastic subtype and poor survival (187). However, only $\sim 5-15 \%$ of medulloblastoma show an amplification of the $M Y C$ gene (188) suggesting that other mechanisms account for the increased c-Myc expression. Understanding how $M Y C$ is regulated in medulloblastoma is critical to develop targeted therapies. 
Gene profiling and murine models have revealed that many of the pathways (Wnt, Shh, Myc and Notch) required in neural stem cells (NSC), multipotent cerebellar SCs and lineage-restricted progenitors of the EGL are also aberrantly activated in medulloblastoma $(17,189)$. Inactivation of Ptch in lineage-restricted granule cell progenitors and NSC has been shown to form medulloblastoma tumors $(190,191)$. Experiments in transgenic mice have revealed that expression of c-Myc is essential for normal developmental control of hematopoietic stem cells (HSC) and NSCs $(87,88)$. Likewise, c-Myc is crucial for self-renewal and maintenance of pluripotency in murine ES cells (90). Therefore, c-Myc is an attractive therapeutic target to inhibit medulloblastoma cell growth. miRNAs are negative regulators of gene expression and have been implicated in regulation of cellular processes. Myc regulates miRNA expression and is also regulated by miRNAs $(176,192,193)$. We intend to identify miRNAs that target MYC as a first step toward the development of potent c-Myc inhibitors.

We used a screening method, which employs a two-tiered assay to enable us to identify miRNAs that target $M Y C$ directly. Tier 1 is designed to identify miRNAs that function in a particular pathway and tier 2 is designed to determine which miRNAs targets a specific 3'UTR. We found that miR-33b adversely regulates $\mathrm{c}-\mathrm{Myc}$ through direct binding to the 3'UTR of the MYC gene. Real-Time PCR data suggests that over-expression of miR-33b results in decreased levels of $M Y C$ mRNA. Also, miR-33b over-expression leads to down-regulation of c-Myc protein expression, and reduces c-Myc-mediated cellular proliferation, cell cycle progression, colony formation and cell migration. Furthermore, 
miR-33b expression in D283-Med (D283) cells reduces neurosphere formation.

Orthotopic injection of mice with miR-33b-expressing D283 resulted in decreased in vivo tumorigenesis. These results demonstrate that miR-33b is able to inhibit cell proliferation, cell cycle progression and suppress medulloblastoma tumor growth.

\subsection{MATERIALS AND METHODS}

\subsubsection{Cell culture}

HEK293T cells were purchased from ATCC and cultured in Dulbecco's modified eagle's medium (DMEM) (Gibco, Carlsbad, CA) supplemented with $10 \%$ heat inactivated fetal bovine serum (FBS) and 0.1\% (v/v) Antibiotic-Antimycotic (Cellgro, Manassas, VA). Rat fibroblasts Rat1c-Myc-/- (clone HO15.19), (from Dr. Claycomb's Lab, Brown University) were maintained in DMEM (Gibco, Carlsbad, CA) supplemented with $10 \%$ fetal bovine serum (FBS) (Gibco, Carlsbad, CA) and $0.1 \%$ (v/v) AntibioticAntimycotic (Cellgro, Manassas, VA). Medulloblastoma cell lines, D283 and DAOY, were purchased from ATCC and cultured in EMEM (Gibco, Carlsbad, CA) supplemented with $10 \%$ FBS and $0.1 \%$ (v/v) Antibiotic-Antimycotic (Cellgro, Manassas, VA). For neurosphere formation, D283 cells were cultured in neurobasal media supplemented with 10\% FBS (Gibco, Carlsbad, CA), 0.1\% (v/v) antibiotic-Antimycotic (Cellgro Manassas, VA), $2 \mathrm{mM}$ L-glutamine, N2 supplement, B27 supplement, $20 \mathrm{ng} / \mathrm{ml} \mathrm{hrEGF}, 20 \mathrm{ng} / \mathrm{ml}$ hrbFGF and $50 \mu \mathrm{g} / \mathrm{ml} \mathrm{BSA} \mathrm{(Invitrogen,} \mathrm{Carlsbad,} \mathrm{CA).} \mathrm{All} \mathrm{cell} \mathrm{lines} \mathrm{were} \mathrm{cultured} \mathrm{at}$ $37^{\circ} \mathrm{C}$ in an atmosphere containing $5 \% \mathrm{CO}_{2}$. 
Transient transfection was performed using Lipofectamine LTX (Invitrogen, Carlsbad, CA). Transient transfection efficiency for $293 \mathrm{~T}$ cells was $>90 \%$ as judged by a control vector, pSIF, expressing green fluorescent protein (GFP). Efficiency for other cells lines was determined based on the expression of GFP from the parental pSIF vector and was recorded to be between $60-90 \%$. For miRNAs, we used the parental vector pSIF as a vector control. For inhibitor of miR-33b, we used Anti-miR ${ }^{\mathrm{TM}}$ miRNA inhibitor (Ambion Inc, Austin, TX), which is chemically modified, single stranded antisense RNA to miRNA designed to specifically bind and inhibit the endogenous miR33b molecules. The negative control (Ambion, Inc., Austin, TX) used for the inhibitor is a random-sequence RNA molecule that has been extensively tested in many human cell lines and tissues and validated to not produce any identifiable effect on known miRNA function.

Stable transfection was performed using viruses generated in 293T cells. For lentivirus production, $293 \mathrm{~T}$ cells was transiently co-transfected, $24 \mathrm{hrs}$ post plating in 6 -well plates, with $2 \mu \mathrm{g}$ of pSIF vector, or miRNA, $1.4 \mu \mathrm{g}$ of $\mathrm{pVGV}-\mathrm{S}$ and $0.7 \mu \mathrm{g}$ of $\mathrm{pFIV}-34 \mathrm{~N}$ packing and expression vectors respectively using Lipofectamine LTX. For retrovirus production, $293 \mathrm{~T}$ cells in 6-well plates were transfected with $2 \mu \mathrm{g} \mathrm{pCL-Eco} \mathrm{and} \mathrm{the} \mathrm{vector} \mathrm{of} \mathrm{interest}$ (pMX-EGFP as control and pMX-gene $\mathrm{X}$ for experiments). The lentivirus-containing or retrovirus containing media was collected after $48 \mathrm{hrs}$. The viral media was supplemented with polybrene (final concentration $5-8 \mu \mathrm{g} / \mathrm{ml}$ ), a polycation that neutralizes charge interaction to increase binding of the virus capsid to the cellular membrane, and used to infect desired cells. The media was changed after $12-24 \mathrm{hrs}$ to minimize the toxic effect 
of polybrene on cell viability. The efficiency of stable transfection was determined based on expression of GFP or RFP by fluorescent microscopy.

\subsubsection{Cloning}

MYC 3'UTR primers were designed using the Vector NTI software: forward primer 5'ATTCTAGAGGAAAAGTAAGGAAAACGATTCCT-3' and reverse primer 5'TAGCGGCCGCGGCTCAATGATATATTTG-3'. The MYC 3'UTR primers were modified at both ends to carry Not1 and Xbal sites. The primer set was used to amplify the 3'UTR of MYC by PCR in a mixture of $0.5 \mu \mathrm{l}$ of $100 \mu \mathrm{M}$ of each primer, $25 \mu \mathrm{l}$ of $2 \mathrm{x}$ buffer H, $2.5 \mu 1$ of Taq polymerase (Promega, Madison, WI), $0.5 \mu 1$ of $10 \mathrm{mM}$ dNTPs (Promega, Madison, WI) and $100 \mathrm{nM}$ of A459 genomic DNA template. The amplified 3'UTR (467bp) was purified from a 1\% agarose gel using Ultrafree-DA columns (Millipore Inc., Billerica, MA) and cloned into pGEM-T vector (Promega, Madison, WI). The resultant pGEM-T-MYC 3'UTR WT was digested with Not//XbaI (New England BioLabs, Ipswich, MA) and cloned into the Not1 and Xba1 site of pRL-TK vector.

The following primer set: reverse 5' GGC AGT TTA AGC CAT GGC TAA 3'; forward primer 5'-ATTCTAGAGGAAAAGTAAGGAAAACGATTCCT-3'; mutant primer 5'GGCAGTTTAAGCCATGGCTAA3'; was used to clone the full mutant MYC 3'UTR, mutated at the miR-33 'seed' binding site, into the Xba1/Not1 sites of pRL-Tk vector in 2 PCR runs. In the first PCR the WT forward primer and the mutant primer were used to amplify an approximately $150 \mathrm{bp}$ product. The first PCR product was used as a forward primer for the second PCR, with the WT reverse primer to amplify the full length mutant 
MYC 3'UTR. The product was purified using the Ultrafree-DA column (Millipore Inc., Billerica, MA) and cloned into pGEM-T vector (Promega, Madison, WI). This resultant pGEM-T-MYC 3'UTR mutant was cut with Not1 and XbaI and cloned into the Not1/XbaI site of pRL-TK vector. Likewise, the following primer set: reverse 5' GGC AGT TTA AGC CAT GGC TAA 3'; forward primer 5'-

ATTCTAGAGGAAAAGTAAGGAAAACGATTCCT-3'; mutant forward primer 5' GTACCTAAGCGGCTAGGTACTA 3' was used to clone mutant MYC 3'UTR mutated at the miR-369-5p 'seed' binding site into the Xba1/Not1 site of pRL-TK.

The oligonucleotides listed in Table 1 were used to clone the miR-33b mutant construct. The oligonucleotides were phosphorylated in a mixture containing $5 \mu \mathrm{l}$ of $10 \mu \mathrm{M}$ of each oligonucleotide (except for 1 and 9), $1 \mathrm{X}$ of T4 polynucleotide kinase (PNK) buffer, $0.5 \mu \mathrm{l}$ of T4 PNK (New England BioLabs, Ipswich, MA), and of $1 \mu \mathrm{M}$ ATP at $37^{\circ} \mathrm{C}$ for 30minutes. PNK was inactivated by incubating at $65^{\circ} \mathrm{C}$ for 5 minutes. The oligonucleotide was then annealed by heating at $90^{\circ} \mathrm{C}$ for $5 \mathrm{mins}$ and eventual cooling to room temperature. The annealed product was adenylated by incubating with dATP at $72^{\circ} \mathrm{C}$ for 30 mins. The resultant pGEM-T-miR-33b mutant construct, as well as pSIF vector, were digested with $\mathrm{MfeI}$ and BamHI and the desired bands, $150 \mathrm{bp}$ and $7 \mathrm{~kb}$ respectively, purified with the Ultrafree-DA column (Millipore Inc., Billerica, MA). The mutant miR-33b insert was then ligated into pSIF at a ratio of 3:1 and confirmed based on band sizes following Mfe1/BamHI restriction enzyme digest. 


\begin{tabular}{|c|c|}
\hline Number & Oligonucleotide \\
\hline 1 & 5'-GGATCCGTGGCGGGTGTGGGGGCCGA-3' \\
\hline 2 & 5'-GGGGCCGCCCGCCTCTCGGCCCCCACACCCGCCACGGCTC-3' \\
\hline 3 & 5'-GAGAGGCGGGCGGCCCCGCGGT-3 \\
\hline 4 & 5'-TCACACACGTGCAATGCAACTGCAATGCACCGC-3' \\
\hline 5 & $\begin{array}{l}\text { 5'- } \\
\text { GCATTGCAGTTGCATTGCACGTGTGTGGGCGGGTGCAGTCCTCG- } \\
\text { 3' }\end{array}$ \\
\hline 6 & 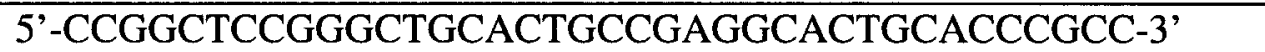 \\
\hline 7 & 5'-GCAGTGCAGCCCGGAGCCGGCCCCTGGCACCACGG-3' \\
\hline 8 & 5'-CAATTGAGGATGGGGGCCCGTGGTGCCAGGGG-3' \\
\hline 9 & 5'-GTTAACTCCTACCCCCG-3' \\
\hline
\end{tabular}

Table 1: Oligonucleotide for miR-33b mutant cloning

The pcDNA3-MYC construct was purchased from Addgene (Cambridge, MA). This construct lacks the MYC 3'UTR but carries the $M Y C$ coding region. We then cloned constructs that either carried the MYC wild-type or mutant 3'UTR. pRL-TK-MYC 3'UTR, pRL-TK-MYC 3'UTR mutant and pcDNA3-MYC constructs were digested with HindIII and Xbal to generate desired bands of $\sim 500 \mathrm{bp}$ (pRL-TK-MYC 3'UTR, pRL-TK-MYC 3'UTR mutant) and 5kb (pcDNA3-MYC). The ligation experiment was set at 1:3 (insert to backbone construct) to generate the final pcDNA3-MYC 3'-UTR (MYC 3'UTR) WT and MYC 3'-UTR mutant constructs. 
All constructs were confirmed by DNA sequencing.

\subsubsection{Luciferase assay}

E2 promoter binding factor (E2F) was originally identified as a cellular transcription factor which could bind to and activate the adenovirus E2 promoter (194). E2F activity appears to play a critical role in cell growth regulation through the activation of a variety of genes that encode proteins important for DNA replication in S-phase, as well as various genes that encode cell cycle regulatory proteins that facilitate the transition through the cell cycle $(195,196)$. The $E 2 F 2$ promoter has 4 distinct E-boxes to which cMyc can bind to regulate E2F2 protein expression (197). pSIF, the parental vector for all our miRNA constructs, served as the control. Renilla luciferase (Rluc) from pRL-TK for tier-1 and luciferase (Luc) reporters from the pGL3-Promoter for tier-2, are used to normalize transfection efficiency and total protein synthesis. If a miRNA directly targets the MYC gene, both Luc/Rluc of the tier-1 assay and Rluc/luc of the tier- 2 assay will be down-regulated compared to respective controls. However, if a miRNA indirectly downregulates c-Myc, only Luc/Rluc expression in the tier-1 assay is likely to be reduced. The success of this strategy depends on: (i) a cell line with a proven record in a specific pathway; (ii) endogenous miRNA expression levels may dictate how many fold the exogenous construct can over-produce a specific miRNA, which can be addressed by comparing levels in cells with or without the miRNA construct, or using antisense to miRNA to inhibit endogenous expression. 
We used a two-tier screening assay: tier-1 is designed to identify any miRNA that modulates the c-Myc signaling pathway, and tier-2 is designed to determine which miRNAs binds the MYC 3'UTR. In tier-1, we made use of an E2F2-Luciferase construct (E2F2-Luc). This construct is made up of a luciferase gene whose expression is regulated by the E2F2 promoter cloned down-stream of the luciferase gene. In this case miRNA modulation of endogenous c-Myc activity regulates firefly luciferase activity. The given miRNA can target c-Myc expression by either binding the 3'UTR of MYC or the 3'UTR of other genes that regulate c-Myc expression. Based on endogenous c-Myc expression, differential binding to the E2F2 E-box occurs, and this is determined based on the luciferase reporter measurement. The pRL-TK (renilla luciferase -Rluc) expression vector is used as an internal control. HEK293T cells $\left(3 \times 10^{4}\right.$ cells/well) were cotransfected in triplicate wells with $80 \mathrm{ng} /$ well of pSIF, or $\sim 400$ miRNA present in our miRNA library, $20 \mathrm{ng} /$ well of pRL-TK and $100 \mathrm{ng} /$ well of the E2F2-Luc construct using the Lipofectamine LTX reagent (Invitrogen, Carlsbad, CA). Luciferase reporter activity was measured $48 \mathrm{hrs}$ post-transfection using the Dual-Glo Luciferase assay system (Promega, Madison, WI) as per the manufacturer's instruction. To determine the effect of a specific miRNA on the c-Myc pathway, the ratio of Luc/Rluc was calculated. The value from the vector-transfected wells was normalized to a value of one, and the values from cells transfected with miRNAs were analyzed in comparison to this. Values lower than one indicated a negative effect on the c-Myc pathway. Similarly, the Dual Glo Luciferase assay was used to determine whether mutation of the E2F2 WT construct abrogated the effects of miRNA targeting. The E2F2-luc mutant construct has mutated bases in two of the E2F2 promoter E-boxes. These mutations have been shown to significantly affect c- 
Myc activity on the E2F2 promoter resulting in reduced luciferase reporter activity (197). This mutant construct (100ng/well) was co-transfected with $80 \mathrm{ng} /$ well of miR-33b, miR$33 \mathrm{a}$ or $\mathrm{pSIF}$, and $20 \mathrm{ng} / \mathrm{well}$ of pRL-TK vector into $293 \mathrm{~T}$ cells $\left(3 \times 10^{4}\right.$ cells/well) in 96well plates. Dual Glo luciferase assay was performed $48 \mathrm{hrs}$ later. Analysis was performed as described for the wild type E2F2-luc construct assay.

Tier-2 assay was designed to determine miRNAs that directly bind the 3'UTR of target genes. In this assay, we used a cloned pRL-TK-MYC 3'UTR-Luc construct and a pGL3Luc construct as a transfection control. In this system, binding of miRNA to the 3'UTR mediates the down-regulation of Rluc expression. We co-transfected $293 \mathrm{~T}$ cells $\left(3 \times 10^{4}\right.$ cells/well) in 96-well plates with $120 \mathrm{ng} /$ well of pRL-TK-MYC-3'-UTR WT, $80 \mathrm{ng} /$ well of 56 miRNAs (individually) predicted from TargetScan and Miranda to target $M Y C$, and $20 \mathrm{ng} / \mathrm{well}$ of pGL3-Luc construct (which act as internal luciferase control) in triplicate wells. Dual-Glo Luciferase assay (Promega, Madison, WI) was performed and miRNAs binding the MYC 3'UTR were determined based on Luc/Rluc ratio. The value obtained from vector-transfected wells were normalized to one and the values for the miRNAs were analyzed in comparison to this, with values lower than one indicating binding to MYC 3'UTR and negative regulation of MYC. A pRL-TK-MYC-3'UTR mutant construct, mutated at 4 nucleotides that form complementary base pairs with the miR-33b seed region, was used to determine whether preventing miR-33b binding at the seed region will abrogate down-regulation of the luciferase reporter. $293 \mathrm{~T}$ cells $\left(3 \times 10^{4}\right.$ cells/well $)$ in 96-well plates were co-transfected with $120 \mathrm{ng} /$ well of pRL-TK-MYC 3'UTR mutant, 80 $\mathrm{ng} /$ well of miR-33b and $20 \mathrm{ng} /$ well of pGL3-Luc construct (internal luciferase control) in 
triplicate wells, and Dual-Glo Luciferase assay was performed. The data were analyzed similarly to that of the WT construct.

\subsubsection{Western blot analysis}

HEK293T cells $\left(1 \times 10^{6}\right.$ cells/well $)$ cultured in 6-well plates were transfected with $2.5 \mu \mathrm{g}$ of pSIF, miR-33b, and miR-33a or miR-333b mutant. Medulloblastoma cell lines DAOY and D283-Med were stably transfected with pSIF-mKate or miR-33b. Total protein was extracted using 1X RIPA reagent (Cell Signaling Technology, Danvers, MA) containing $1 \mathrm{X}$ protease inhibitor cocktail (Sigma, St. Louis, MO). The protein concentration was determined using the Bicinchoninic Acid (BCA) method (Thermo scientific, Rockford, IL) according to the manufacturer's protocol. The sample was boiled with Leammli buffer for 10 mins, fractionated by SDS-polyacrylamide gel electrophoresis and transferred at $4^{\circ} \mathrm{C}$ to nitrocellulose membranes. Protein membranes were incubated in blocking buffer (1x Tris-buffered saline - TBS, ph 7.5, 5\% Non-fat dried milk) for $2 \mathrm{hrs}$ at room temperature. The membranes were incubated over-night at $4^{\circ} \mathrm{C}$ on a shaker with anti-c-Myc (Santa Cruz, Santa Cruz, CA; sc-40; diluted 1:1000), anti-Cyclin E (Santa Cruz, Santa Cruz, CA, ; sc-25303; diluted 1:1000), anti-VEGF-A (Santa Cruz, Santa Cruz, CA; sc-7269; diluted 1:1000), anti-Gadd45 (Santa Cruz, Santa Cruz, CA; sc-6850; diluted 1:1000), anti-ODC (Cell signaling, Danvers, MA: 1:1000) or $\beta$-Actin (Sigma, St. Louis, MO; AC-15; diluted 1:5000) antibodies. The membranes were washed 3 times, 10 minutes per wash, with $1 x$ TBS containing $0.05 \%$ Tween 20 , incubated with horse peroxidase-linked goat anti-mouse IG antibody (Santa Cruz, Santa Cruz, CA; sc-2005; 1:5000) or goat anti-rabbit IG antibody (Cell Signaling, Danvers, 
MA) for $1 \mathrm{hr}$ at room temperature, washed $3 \mathrm{X}$ for $10 \mathrm{mins}$, and visualized with the Supersignal west Dura/Femto Chemiluminescent substrate kit (Pierce, Rockford, IL).

c-Myc null cells, HO15.19 ( $2 \times 10^{6}$ cells/ well) were plated in 6-well plates and cotransfected with $2 \mu \mathrm{g}$ pSIF, miR-33b or miR-33a, and $2 \mu \mathrm{g}$ of pcDNA3.1-MYC-MYC 3'UTR or pcDNA3.1-MYC-MYC 3'-UTR mutant. Total protein was extracted $48 \mathrm{hrs}$ after transfection and western blot analysis performed to determine c-Myc, and Cyclin E and $\beta$-Actin expression.

The quantification of protein expression levels was determined by ImageJ (http://rsb.info.nih.gov/ij) with arbitrary units (AU) reflecting the signal density from the blot.

\subsubsection{Quantitative real-Time PCR (qRT-PCR)}

TaqMan miRNA assay and gene expression assay (applied Biosystem inc., Forest City, CA) were used to detect the expression level of mature miRNAs and gene mRNA expression, respectively (198). U6 RNA and $\beta$-Actin mRNA was used for normalization. To determine whether miR-33b affects MYC mRNA levels, $293 \mathrm{~T}$ cells $\left(5 \times 10^{4}\right.$ cells/well), were plated in 12-well plates and transfected with $1 \mu \mathrm{g}$ of pSIF, miR-33b, miRNA inhibitor negative control or antisense miR-33b. Also, D283 and DAOY cells were stably infected with empty vector or miR-33b. Total RNA was extracted $48 \mathrm{hrs}$ posttransfection by one of two methods: the cells were washed twice with 1X PBS, resuspended in $50 \mu \mathrm{l}$ of $1 \mathrm{X} \mathrm{PBS}$ and heated at $95^{\circ} \mathrm{C}$ for 5 mins; or total RNA was 
extracted using TriZol reagent (Invitrogen, Carlsbad, CA) as per the manufacturer's protocol. Total RNA concentration was detected by nanodrop. To quantify miR-33b expression levels, reverse transcription (RT) reaction was performed with 100 ng RNA in a reaction mixture containing $5 \mathrm{x}$ miR-33b and U6 RT primer, $1 \mathrm{X}$ RT buffer and $0.0125 \mathrm{mM}$ DTT. For mRNA expression level, reverse transcription (RT) reaction was performed with $100 \mathrm{ng}$ RNA using the iScript cDNA synthesis Kit (Bio-RAD, Hercules, CA) as per the manufacturer's instruction. Reverse transcription conditions were as follows: $16^{\circ} \mathrm{C}$ for $30 \mathrm{mins}, 42^{\circ} \mathrm{C}$ for 30 mins and $85^{\circ} \mathrm{C}$ for 5 mins. The PCR product was used in a TaqMan PCR miRNA or gene expression assay (ABI, Forest City, CA). TaqMan 20x probe for miR-33b was used to detect expression levels of miR-33b and TaqMan 20x probe for $M Y C$ was used to detect expression levels of $M Y C$ mRNA. U6 RNA or $\beta$-Actin mRNA was used for normalization. Similar experiments were done with total RNA extracted from $M Y C$ null cells HO15.19, co-transfected with; $1 \mu \mathrm{g}$ of pSIF, miR-33b or miR-33a and $1 \mu \mathrm{g}$ of pcDNA-MYC-MYC 3'-UTR WT or pcDNA-MYC-MYC 3'-UTR mutant.

To determine the expression level of stem cell marker genes, TaqMan gene expression assay was performed from total RNA extracted from neurospheres and differentiated neurospheres. D283 cells, stably transfected with vector or miR-33b and cultured in neurobasal media, formed neurospheres that were further differentiated by culturing in EMEM growth media. Total RNA was extracted from neurospheres or differentiated neurospheres using TriZol reagent. cDNA was synthesized using the iScript kit and used 
for TaqMan real-time PCR for $M E L K, M Y C, S O X 2, C D 133$ and $\beta$-Actin mRNA quantification.

To determine whether c-Myc activates the miR-33b promoter, 293T cells and HeLa cells $\left(1 \times 10^{4}\right.$ cells/well) were plated in 24-well plates and transfected with $3 \mu \mathrm{g}$ of pcDNA3.1+, or 1,2 , or $3 \mu \mathrm{g}$ of pcDNA-MYC, and total RNA extracted after $48 \mathrm{hrs.}$ QRTPCR was performed using the TaqMan gene or miRNA expression assay for quantification of MYC mRNA and miR-33b levels. $\beta$-Actin and $U 6$ were used as internal controls.

\subsubsection{Cell cycle analysis}

D283 and Daoy cells were stably transfected with empty vector or miR-33b. Also, Myc null cells, HO15.19, were co-transfected with vector or miR-33b and pcDNA-MYC 3'UTR or pcDNA-MYC 3'UTR Mut. The various cell types were collected after $48 \mathrm{hrs}$ and washed twice in 1X PBS. Cells were suspended in 1 $\mathrm{ml}$ of ice-cold $70 \%$ ethanol and kept at $4^{\circ} \mathrm{C}$ for $24 \mathrm{hrs}$. The next day, the cells were washed twice with 1X PBS and treated with $1 \mathrm{mg} / \mathrm{ml} \mathrm{Ribonuclease} \mathrm{for} 90$ mins at $37^{\circ} \mathrm{C}$ in $0.5 \mathrm{ml}$ of $1 \mathrm{X}$ PBS. Before cell cycle analysis, cells were stained with $5 \mu \mathrm{g} / \mathrm{ml}$ of PI and protected from light. Flow cytometry was performed with a FACScan apparatus. A minimum of 20,000 cells per sample were collected and the FACS files were further analyzed using FlowJo software (Tree Star Inc., Ashland, OR) for cell cycle analysis. The excitation wavelength was $488 \mathrm{~nm}$, and red fluorescence ( $>590 \mathrm{~nm}$ for PI) was recorded. In addition, the parameters 
for forward scatter (FSC) and side scatter (SSC) were determined. For each variable (exposure condition, culture period etc.) a minimum of 3 samples were quantified.

\subsubsection{Transwell cell migration assay}

D283 $\left(1 \times 10^{4}\right.$ cells/well $)$ and Daoy $\left(1 \times 10^{4}\right.$ cells/well $)$ cells were stably transfected with $1.6 \mu \mathrm{g}$ of empty vector or miR-33b, washed twice with $1 \mathrm{X}$ PBS and resuspended in serum-free EMEM. Transwell (Boyden) assay was then performed $48 \mathrm{hrs}$ after transfection. Briefly, Transwell (Boyden) chambers (Invitrogen, Carlsbad, CA), with a pore size of $8 \mu \mathrm{m}$, were placed into triplicate 12 -well plates. EMEM media supplemented with $10 \%$ FBS which served as a chemoattractant was added to wells beneath the Transwell chamber. D283 cells $\left(1 \times 10^{4}\right)$ or Daoy cells $\left(1 \times 10^{4}\right)$, in $0.5 \mathrm{ml}$ of serum-free EMEM media were added to the Transwell chamber and the plates were then incubated at $37^{\circ} \mathrm{C}$ in a humidified $5 \% \mathrm{CO}_{2}$ atmosphere for $24 \mathrm{hrs}$. The cells were fixed with methanol for 10 mins, and stained with $0.4 \%$ crystal violet for $2 \mathrm{hrs}$. Non-migrated cells on the upper side of the filter were removed with a cotton swab, and the filter mounted on microscope glass slides. Images were captured using a Nikon Eclipse TE300 microscope and a Nikon Plan Fluor 10x0.30 objective. The migrated cells were counted using ImageJ software. D283 and Daoy cells $\left(0.5 \times 10^{4}\right)$ similarly transfected, were cultured in serum free media, and 24hrs later MTT cell proliferation assay performed.

\subsubsection{Neurosphere assay}

The neurosphere assay has proven to be an excellent technique to isolate neural stem cells and progenitor cells to investigate the differentiation and potential of cell lineages. 
Medulloblastoma is believed to have a stem cell origin; therefore we reasoned that growth of medulloblastoma cells in media that favors neural stem cell growth will result in neurosphere formation. D283 cells $\left(1 \times 10^{6}\right)$ were stably transfected with empty vector or miR-33b and neurosphere assay performed. Briefly, the cells were trypsinized, washed in neurobasal medium (Invitrogen, Carlsbad, CA) and resuspended in Matrigelcoated plates at $5 \times 10^{4}$ cells $/ \mathrm{ml}$ in the neurobasal medium containing $2 \mathrm{mM} \mathrm{L}$-glutamine, N2 supplement, B27 supplement, 20ng/ml hrEGF, 20ng/ml hrbFGF and 50 $\mu \mathrm{g} / \mathrm{ml}$ BSA (Invitrogen, Carlsbad, CA). The growth factors, 20ng/ml hrEGF and 20ng/ml hrbFGF, were added daily to the plates and the neurobasal medium were replaced twice per week with fresh neurobasal media. After 10days of incubation, primary neurospheres were disaggregated into single-cell suspensions and reseeded to form secondary, tertiary and quaternary spheres in 96-well plates. Spheres larger than $50 \mu \mathrm{m}$ in diameter were counted by microscopy (magnification, 10X) and quantified.

\subsubsection{Soft agar colony formation (clonogenic) assay}

Clonogenic assay or colony formation assay is an In Vitro cell survival assay based on the ability of a single cell to grow into a colony. The assay essentially tests every cell in the population for its ability to undergo "unlimited" division. The effect of miR-33b overexpression on colony formation ability of D283 cells was assessed by soft agar colony formation assay. Medulloblastoma D283 cells were stably transfected with empty vector or miR-33b. The cells were collected in fresh EMEM media after 48hrs and incubated in soft-agar. The soft agar plate contains a bottom layer of $0.5 \%$ agar and a top layer of $0.2 \%$ agar. Approximately 2000 , appropriately transfected, D283 cells were plated over 
the top layer of a 6-well plate. The cells were maintained at $37^{\circ} \mathrm{C}$ in a humidified $5 \% \mathrm{CO}_{2}$ atmosphere for two weeks. Fresh media was added at regular intervals to prevent the

plates from drying out. At the end of two weeks, the cells were treated with $0.5 \%$ of MTT reagent for 2-3 hrs. Colony sizes were assessed after imaging using a scanner or microscopy (original magnification, $\mathrm{x} 4$ ).

\subsubsection{Orthotopic tumor formation}

All animal experiments were conducted according to a protocol approved by the University of Louisville Animal Care and Use Committee (IACUC number 08107). D283 cells $\left(1 \times 10^{6}\right.$ cells/well $)$ in 6 well plates were stably transfected with vector or miR-33b. Positive transfection was checked by visualization of red fluorescent protein (RFP) expressed by the vector and, positive transfectant were selected with $400 \mu \mathrm{g} / \mathrm{ml}$ of G418 (Cellgro). Recipient immunodeficient nude mice were purchased from Taconic (C.B-Igh$1^{\mathrm{b}} /$ IcrTac-Prkdc ${ }^{\text {scid }}$; FOX CHASE CB-17 SCID ICR; Taconic). Male 9- to 12-week old mice were anesthetized with rapid-sequence inhalation isofluorane (Abbot Laboratories, Abbott Park, IL). 10-mm skin incision was made along the midline of the head and a $1 \mathrm{~mm}$ burr-hole was drilled into the skull and a total of $1 \times 10^{5}$ cells expressing the empty vector or miR-33b were orthotopically injected into the cerebellum of each immunodeficient nude mouse. The mice were monitored for any sign of distress and maintained for 6 weeks in the animal housing facility at the University of Louisville. The mice were sacrificed 6 weeks later and fixed in $10 \%$ formalin for 3 days. After fixation, the brains were embedded in paraffin and $5 \mu \mathrm{m}$ thick sections were made following routine procedure. 
H\&E staining was performed to determine size and location of tumors formed by D283 cells either expressing empty vector or miR-33b. Briefly, the tissue sections were deparafinized by incubating twice in xylene for 10 mins per incubation. The sections were then rehydrated by incubating twice for 5 mins in 100\% ethanol, then for 2 mins each in $95 \%$ and $70 \%$ ethanol. The section was then washed with copious amounts of distilled water and stained by incubating in Harris hematoxylin (Sigma, St. Louis, MO) solution for 10 mins, followed by washing with tap water for at least 5 mins. The sections were then differentiated by incubating in $1 \%$ ethanol for 30 seconds and washed with tap water for 2 mins. The sections were then washed and rinsed in 90\% ethanol and counterstained in eosin-phloxine (Sigma, St. Louis, MO) solution for 0.5-1 min. After the staining the section were dehydrated by incubating twice in $95 \%$ ethanol for 5 mins per incubation and 2 changes of xylene for 5 min per change. The sections, from comparison brain location, were then mounted on the microscope using xylene based mounting medium. Images of the tumors or whole brain were taken using a microscope (magnification 20X, 100X, 200X and 400X). Tumor surface area was determined using the Olympus microscope.

\subsubsection{Immunohistochemistry (IHC)}

Immunohistochemistry was performed according to the avidin biotin complex (ABC) peroxidase method using Rabbit and Mouse IgG ABC Elite1 detection kits (Vector Laboratories, Burlingame, CA). Prior to the performance of the immunohistochemical procedure, $5 \mu \mathrm{m}$ thick histological sections from paraffin-embedded tissue blocks were subjected to non-enzymatic antigen unmasking in $0.01 \mathrm{M}$ sodium citrate buffer $(\mathrm{pH} 6.0)$ 
for $10 \mathrm{~min}$ in a microwave at medium power. The rabbit polyclonal antibody against cMyc (Santa Cruz; SC-789; Dilution 1:500), rabbit monoclonal antibody against Sox2 (Cell signaling; D6D9; Dilution 1:500), rabbit monoclonal antibody PC10 to PCNA (Cell Signaling; PC10: Dilution 1:100), and rabbit monoclonal antibody against Cyclin E (Abcam; ab7959: Dilution 1:100) were used to determine protein expression. Briefly, tumor sections on slides were deparaffinized by incubating in HD1 and HD2 solution for 10 mins per solution. This was followed by re-hydration by incubating at: $100 \%, 95 \%$, $80 \%$, and $70 \%$ ethanol for 5 mins per solution, followed by 5 min incubation in 1XPBS. The slides were then washed copiously with $\mathrm{dH}_{2} \mathrm{O}$. The tumor sections were incubated with a proteinase $\mathrm{K}$ for 5 mins and peroxidase block solution for 15 mins at room temperature. Primary antibody against c-Myc, Sox 2 or PCNA was added and the slides were incubated overnight at $4^{\circ} \mathrm{C}$. The slides were then washed three times with $1 \mathrm{X}$ PBS and incubated with secondary anti-rabbit-HRP antibody for $1.5 \mathrm{hrs}$. The slides were then washed three times with 1X PBS at 10 mins per wash. This was followed by chemical reaction of the horse radish peroxidase (HRP) attached to secondary antibody by incubating tissue sections in HRP DAB substrate buffer, followed by counter staining with methyl green or hematoxylin solution. The slides were then washed in $\mathrm{dH}_{2} 0$. The tumor samples were then dehydrated by incubation for 5 mins each in $70 \%, 80 \%, 95 \%$ and $100 \%$ ethanol and the HD1 and 2 solutions for 10 mins each. Tumor slides were imaged by microscopy (magnification, 20X, 40X, 100X, 200X and 400X). The tumor images were scored on a scale of 0 to 3 (3 indicating strong expression, 2 moderate expression, 1 medium expression, 0 no expression) by three independent scorers. 


\subsubsection{Statistics}

The experimental results were expressed as the mean \pm standard deviation of at least 3 independent experiments. Two-tail Student's t-test was performed with $\mathrm{p} \leq 0.05$ (*) considered as statistically significant between the samples and their respective controls.

\subsection{RESULTS}

\subsubsection{MicroRNAs target $M Y C$ 3'-UTR}

We used reporter assays to screen miRNAs for their ability to modulate c-Myc transcriptional function and target $M Y C 3^{\prime} U T R$ directly (Fig. 3). In Assay 1, a firefly luciferase gene (luc) is driven by the promoter to E2F2 (E2F2-luc), which is regulated by c-Myc (197). miRNAs-of-interest, E2F2-luc, and a Renilla luciferase (Rluc) construct constitutively expressing $R l u c$ are co-introduced into 293T cells to screen miRNAs that down-regulate the reporter luc. In Assay 2, we cloned the 3'UTR of MYC downstream of the Rluc construct and co-expressed it with a respective miRNA and a constitutively expressed $l u c$ to determine whether the miRNA targets the MYC 3'UTR to down-regulate Rluc. 


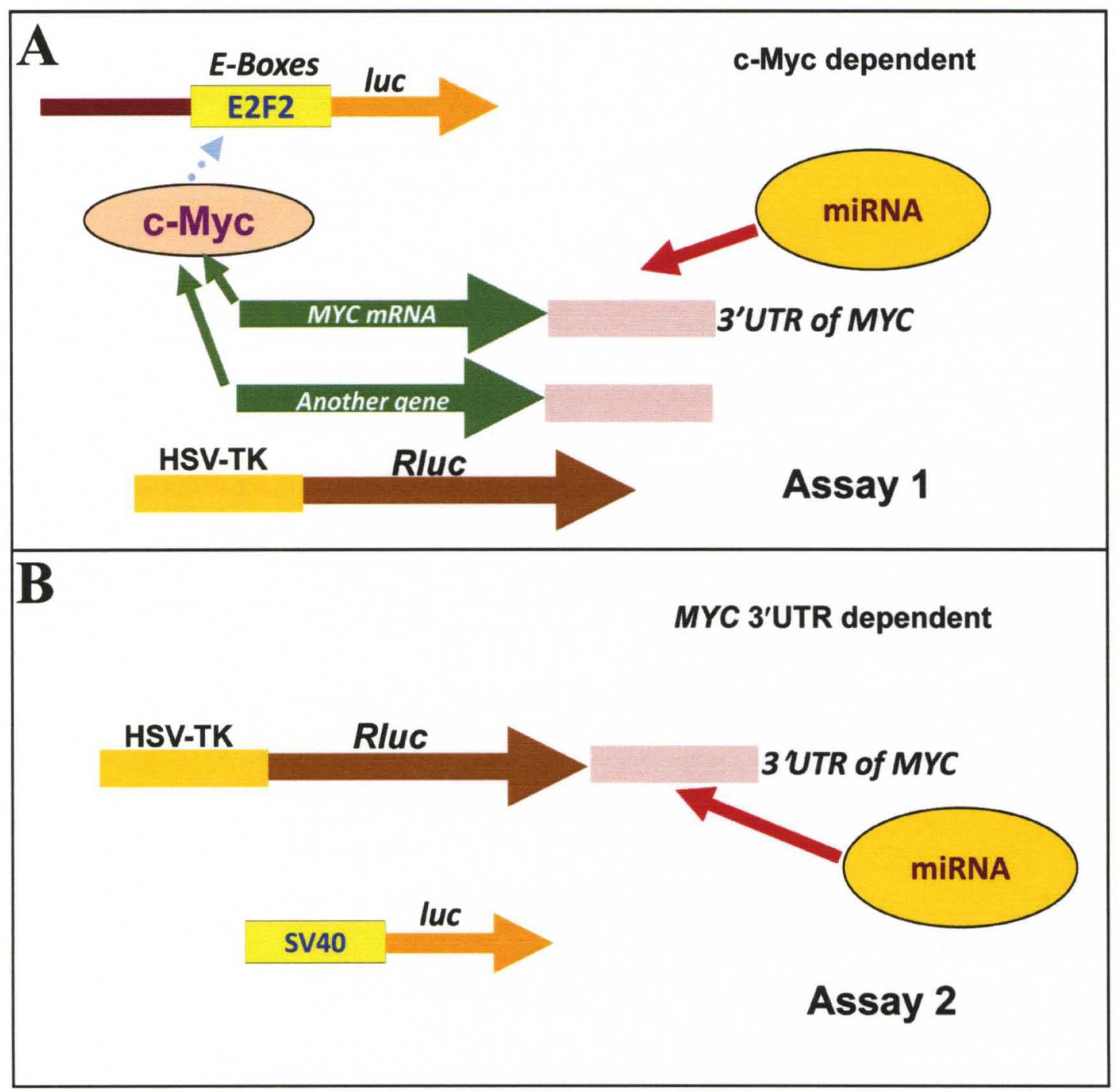

Fig. 3: The 2 tier luciferase screening method. (A) Assay 1 is designed to identify miRNA that functions in the c-Myc pathway and can either target the 3'UTR of $M Y C$ or the 3 'UTR of another gene that regulate c-Myc activity. (B) Assay 2 is designed to identify miRNAs that directly target the 3 'UTR of $M Y C$. The MYC 3'UTR of Myc is cloned down-stream of a renila (Rluc) gene and binding of the miRNA to the 3 'UTR down-regulates the luciferase activity from Rluc. 
We performed Assay 1 in 293T cells using hundreds of miRNA minigenes in our genetic library (199) and found that 4 miRNAs (miR-33a, miR-33b, miR-212, miR-203) significantly down-regulated the c-Myc-dependent reporter, while miR-210 up-regulated it (Fig. 4A). Next, we performed Assay 2 using 54 miRNAs that were predicted to target $M Y C$ and found that miR-33b and miR-203 down-regulated the reporter with MYC 3'UTR downstream (Fig. 4B). Our results with miR-210 suggested that it did not target the 3'UTR of $M Y C$, but up-regulated c-Myc-dependent luc expression, which is consistent with a recent study demonstrating that miR-210 targets MNT, an antagonist of c-Myc (200). To determine whether MYC is a bona fide miR-33b target gene, we performed Assay 1 using a mutant E2F2-luc construct, in which 2 of its 4 E-boxes (cMyc binding sites) were disrupted (197). As expected, the expression of $l u c$ was significantly reduced ( $\sim 10$ fold) when E-boxes on the promoter were perturbed (Fig. 5A). The regulation of $l u c$ by miR-33b was abolished with the removal of 2 E-boxes (Fig. 5A). When a MYC 3'UTR mutation construct (Fig. 5B) that disrupts miR-33b binding to the 'seed' sequence of miR-33b was used in Assay 2, we found that the down-regulation of Rluc by miR-33b was abrogated (Fig. 5B). Our results verified that miR-33b is a negative regulator of c-Myc through directly targeting MYC 3'UTR. 
A

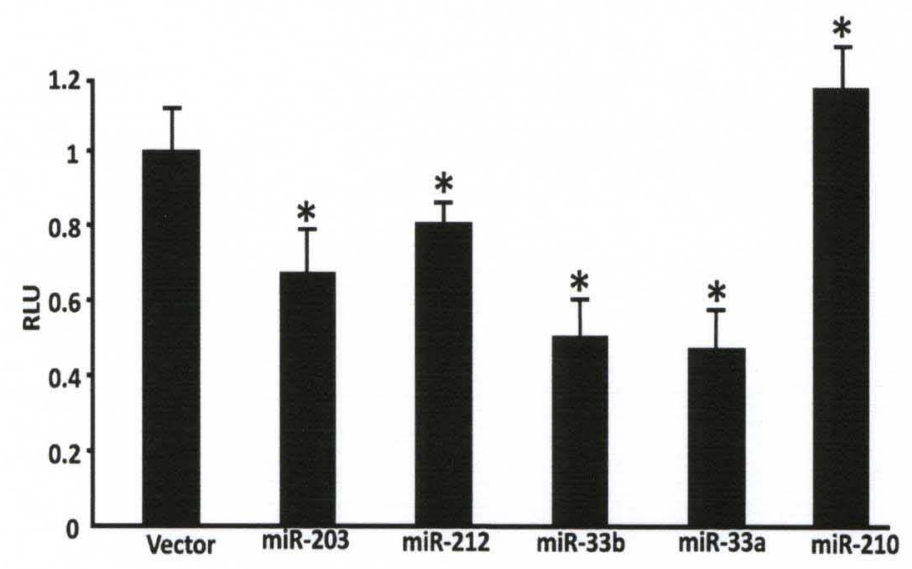

B

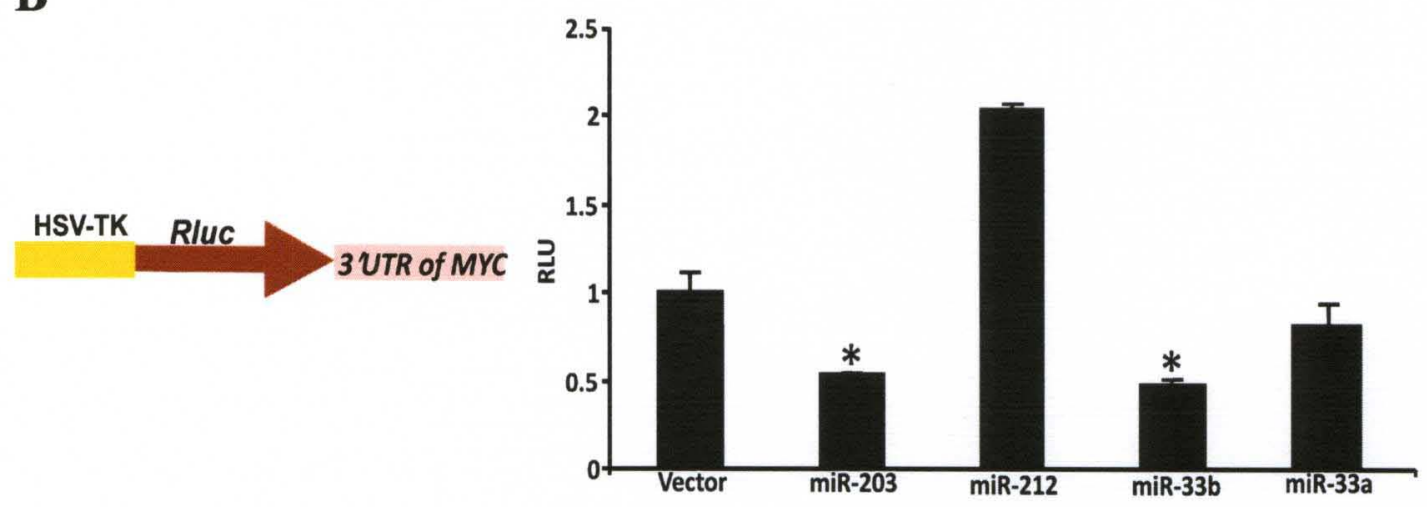

Fig.4: miR-33b is a negative regulator of c-Myc directly targeting the MYC 3'UTR. (A) Luciferase assay from an E2F2-luciferase construct. The Luciferase activity is under the control of the E2F2 promoter that contains $4 \mathrm{c}-\mathrm{Myc}$ binding E-boxes. Values below the RLU of the vector (normalized to 1) indicate downregulation of luciferase activity. (B) Rluc assay from a Myc 3'UTR cloned upstream of the renilla luciferase gene. Values below the RLU of the vector (normalized to 1) indicate down-regulation of luciferase activity. 
A

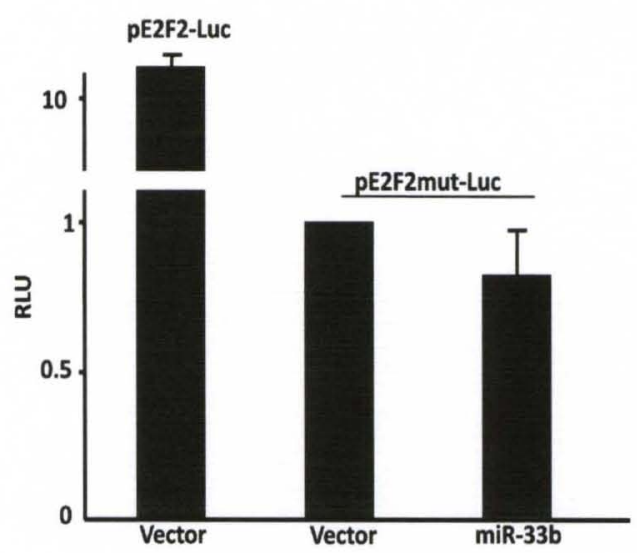

B

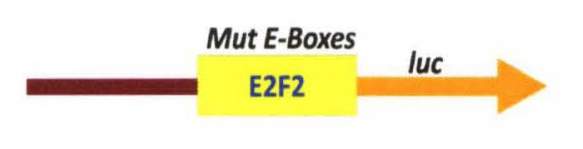

$\begin{array}{ll}\text { MYC3'UTR } & \text { 5'-CAAAUGCAUGAUCAAAUGCAAC-3' } \\ \text { miR-33b } & \text { 3'-CGUUACGUUGUCG-UUACGUG-5' } \\ \text { MYC3'UTR Mut } & \text { 5'-CAAAUGCAUGAUCACCGACAAC-3' }\end{array}$

HSV-TK

Rluc 3 UTR of MYC Mut

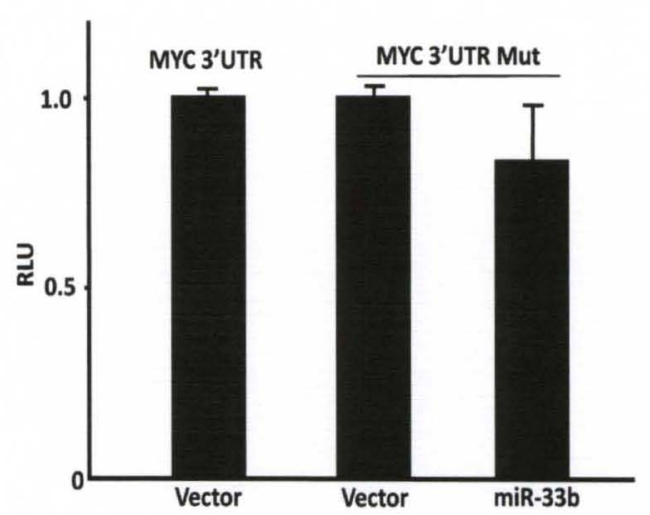

Fig.5: Mutant E2F-luc or MYC 3'UTR are not targeted by miR-33b. (A) Luciferase assay from E2F2-Luciferase mutant construct. Two c-Myc binding E-Boxes are mutated. (B) Luciferase assay from a MYC 3'UTR mutant construct (Right). Mutant construct containing four nucleotides substitution at the miR-33b 'seed' binding site on MYC 3'UTR (Left). 


\subsubsection{Over-expression of miR-33b in 293T, Daoy and D283 cells down-regulates MYC mRNA expression}

We used TaqMan miRNA and gene expression assays to determine which possibility is favored by miR-33b on MYC mRNA. In the scenario where a miRNA leads to mRNA translational repression, the steady state level of mRNA in the cell will remain unchanged despite down-regulation of protein expression, whereas if the miRNA leads to cleavage of the mRNA, down-regulation of the target gene's mRNA will be observed. Following transfection of miR-33b into 293T, D283 and Daoy cells, total RNA was extracted and used for TaqMan qRT-PCR. The miR-33b expression was increased by more than 10 fold following over-expression compared to the parental vector control. The steady state levels of MYC mRNA were significantly reduced with miR-33b over-expression in $293 \mathrm{~T}$ (Fig. 6A), D283 (Fig. 6B) and Daoy cells (Fig. 6C), suggesting that mRNA degradation likely contributed to miR-33b-mediated $M Y C$ suppression. 

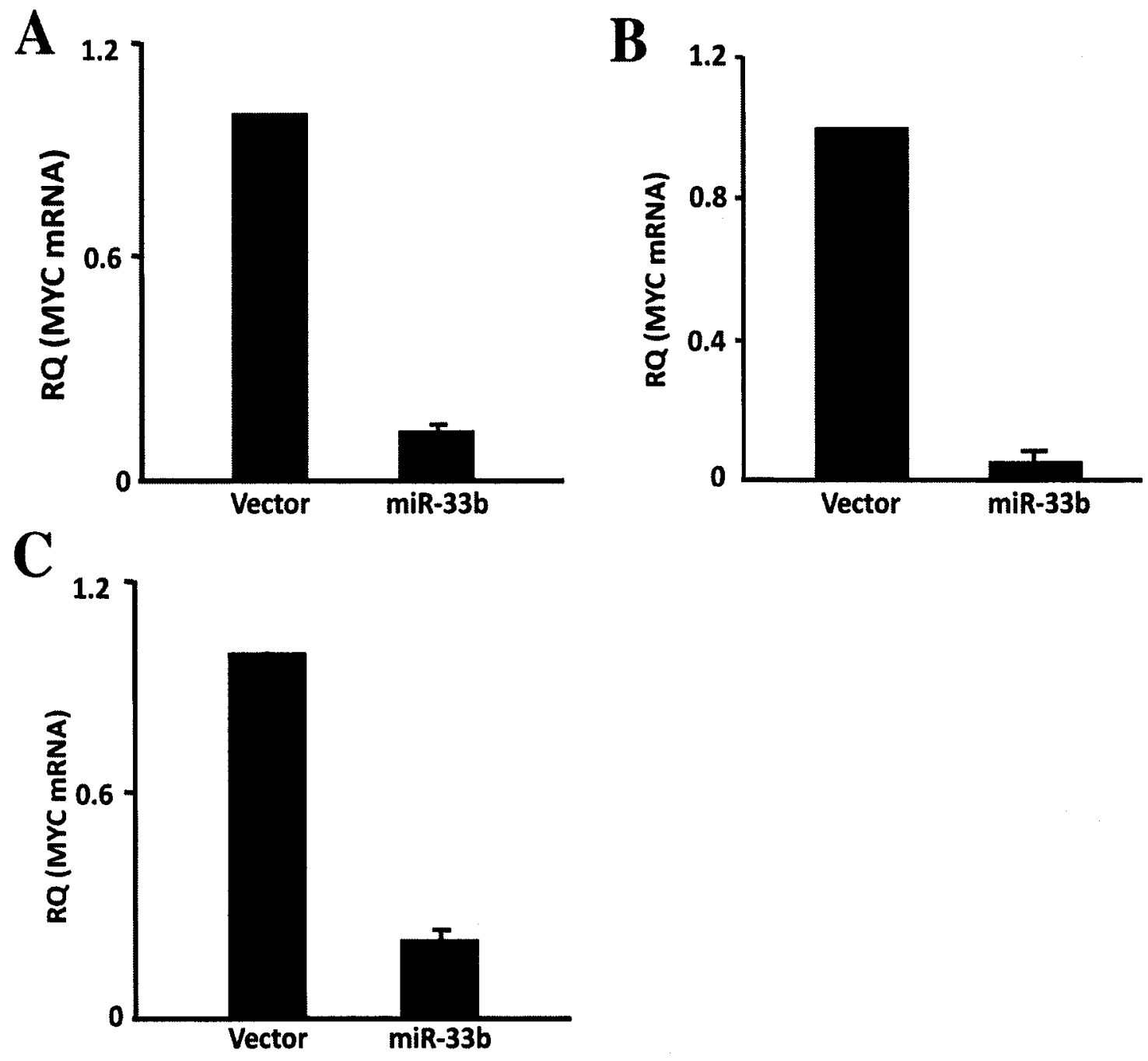

Fig.6: Over-expression of miR-33b results in decreased levels of $M Y C$ mRNA. (A) QRT-PCR shows that $M Y C$ mRNA levels are reduced following transient transfection of miR-33b for 48 hrs. (B) Stable transfection of miR-33b in miR33b-negative medulloblastoma cells results in decreased levels of $M Y C$ mRNA by qRT-PCR. (B) QRT-PCR of MYC mRNA level is reduced in response to overexpression of miR-33b in miR-33b-positive Daoy cells. 


\subsection{3. miR-33b down-regulates the expression of c-Myc protein and its}

\section{transactivational function in $293 \mathrm{~T}$ cells}

To determine whether miR-33b expression result in down-regulation of c-Myc protein expression, we over-expressed miR-33b and performed western blot analysis. We transfected increasing amounts of miR-33b expression constructs $(0,1,2$ and $3 \mu \mathrm{g})$ into 293T cells in 6-well plates and found that over-expression of miR-33b down-regulated cMyc protein levels in a concentration-dependent manner (Fig. 7A). We did not pursue miR-203 as it did not reduce c-Myc protein levels (Fig. 7A). In subsequent experiments, except when stated, we use $2 \mu \mathrm{g}$ of miR-33b expression plasmid to avoid a significant reduction of cell proliferation and unwanted cell death. We noted that miR-33b but not miR-33a down-regulated c-Myc expression (Fig. 4B), although miR-33a differs from miR-33b by only two nucleotides (UA compared to CG respectively) on position 9 and 10 of their mature sequences (Fig. 7B). We constructed a mutant miR-33b (miR-33bM) whose mature sequence resembled miR-33a (Fig. 7B). Over-expression of miR-33b but not miR-33a or miR-33bM reduced the protein levels of c-Myc, as well as two known cMyc transactivational targets: Cyclin E and ornithine decarboxylase (ODC); while Gadd45a, a c-Myc-repressed target was up-regulated (Fig. 7C). The miR-33b binding site is present in MYC 3'UTRs from human, chimpanzee, and rhesus, but not those from mouse, rat, dog, and other mammals (Fig. 8A) and miR-33b is only present in primates (201), suggesting that miR-33b is a primate-specific regulator of c-Myc. Overexpression of an exogenous $M Y C$ gene in 293T cells did not increase the expression levels of miR-33b (Fig. 8B), indicating that miR-33b expression is not regulated by cMyc. 


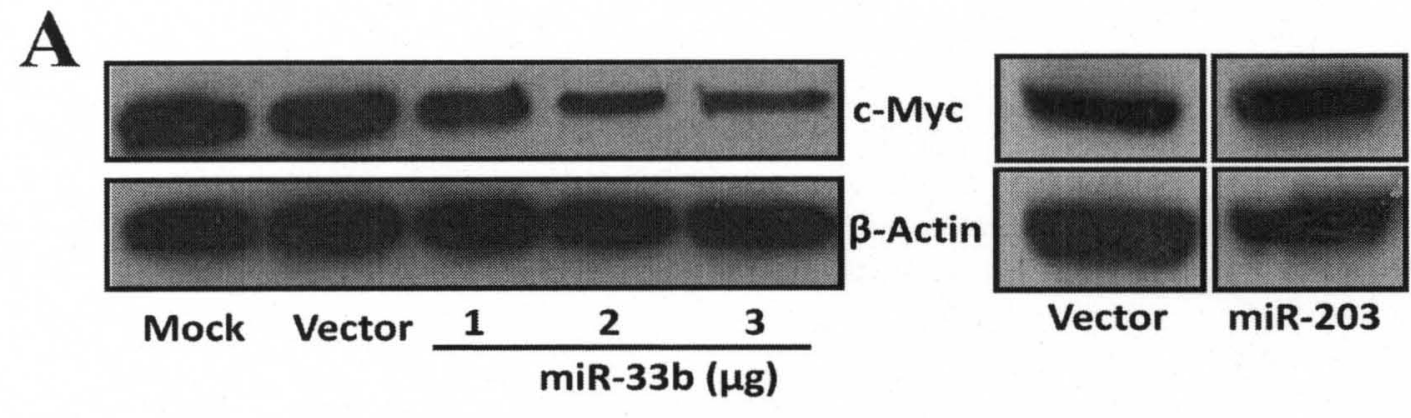

B
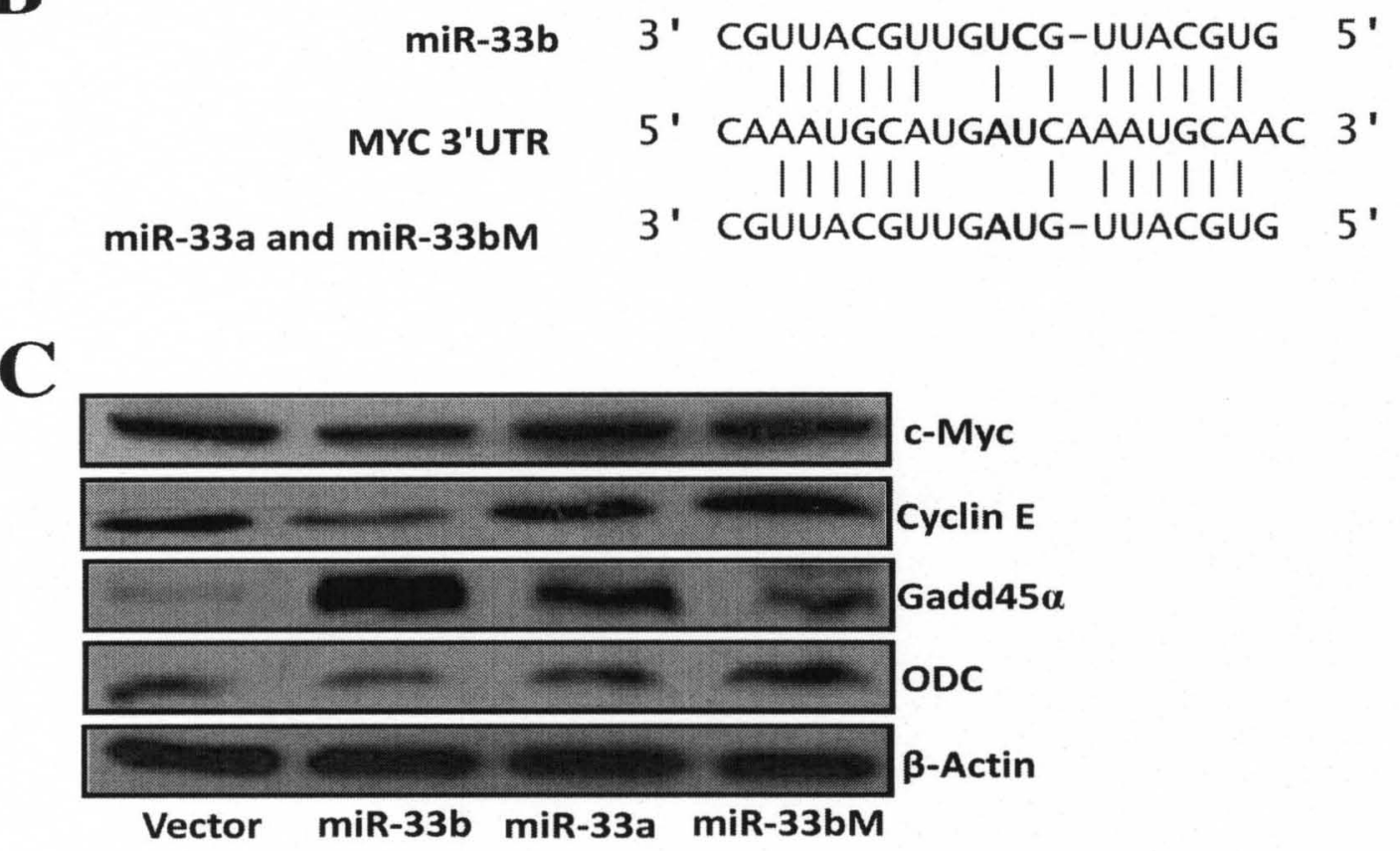

Fig. 7: Over-expression of miR-33b in 293T cells down-regulates c-Myc and its target genes. (A) Western blot analysis for expression of c-Myc in 293T cells transfected with increasing concentration of miR-33b. miR-203 did not affect cMyc protein expression (B) Schematic representation of the binding of miR-33b, miR-33b mutant (miR-33bM) to MYC 3'UTR. (C) Western blot analysis for cMyc, Cyclin E, ODC and Gadd45a levels upon over-expression of miR-33b, miR-33a or miR-33bM in 293T cells. 
A

CGUUACGUUGUCG-UUACGUG 5' hsa-miR-33b

|||||| |||||| 101 UTR

CAAAUGCAUGAUCAAAUGCAACCUCACAACCUUGGCU Human

CAAAUGCAUGAUCAAAUGCAACCUCACAACCUUGGCU

CAAAUGCAUGAUCAAAUGCAACCUCACAACCUUGGCU

- AAAUGCAUGCCAAAGCCUAACCUCACAACCUUGGCU

- AAAUGCAUGCCAAAGCCUAACCUCACAACCUUGGCU

CACAUGCAUGGUCAAGUGCAACCUCACAACCUUGGCU

CAAAUGCAUGUUUAAGUGCAACCUCACAACCUUGGCU

CAAAUGCAUGGUCAAAUGUAACCUCACAACCUUGGCU

CAUAUGCAUGA---- ACAACCUCACAACCUUGGCU

CAACUGCAUGCUAACUUGAGACUACACAACCUUGGCC

Chimpanzee

Rhesus

Mouse

Rat

Rabbit

Dog

Horse

Cow

CAAAUGCAUGaUCAAaUGCAACCUCACAACCUUGGCU

Chicken

Consensus
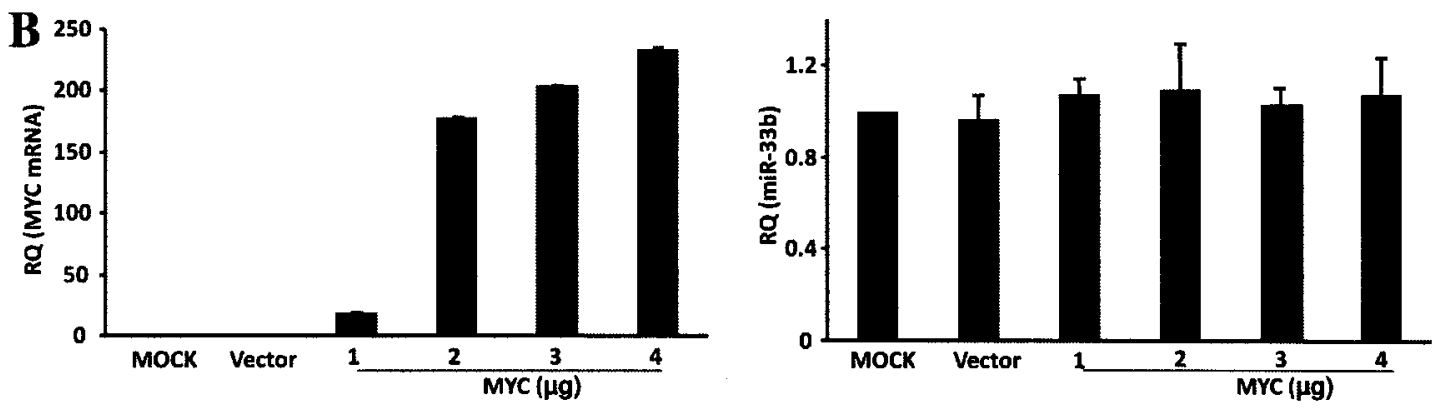

Fig. 8: c-Myc does not regulate miR-33b expression. (A) miR-33b gene exists in human, chimpanzee, and Rhesus, but not in other animals even though they all possess the host gene SREBF1. (B) QRT-PCR for MYC mRNA and miR-33b from 293T cells following transfection with a c-Myc expression vector, pcDNAMYC. The expression of miR-33b remains constant following c-Myc overexpression. 


\subsection{4. miR-33b down-regulates exogenously expressed c-Myc in $M Y C$-null HO15.19}

cells

To further validate that miR-33b specifically targets $M Y C$, we introduced two c-Myc constructs into a MYC-null cell line HO15.19 (202): both have a native c-Myc coding sequence but one with a wild-type 3'UTR (3'UTR WT) and the other with a mutant 3'UTR, in which the miR-33b binding site was disrupted (3'UTR Mut). As shown in Fig. 9A, miR-33b down-regulated the expression of c-Myc and Cyclin $\mathrm{E}$ in cells with 3'UTR WT but not in those with 3'UTR Mut. The changes of MYC mRNA levels showed similar patterns to that of the protein levels (Fig. 9B). miR-33b led to more G1 arrest in cells carrying MYC with 3'UTR WT compared to that with 3'UTR Mut (Fig. 9C). These results show that miR-33b regulation of c-Myc expression and function is dependent on the 3'UTR of MYC mRNA. 
A

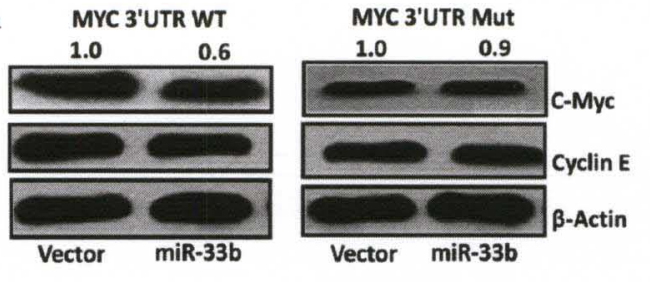

C

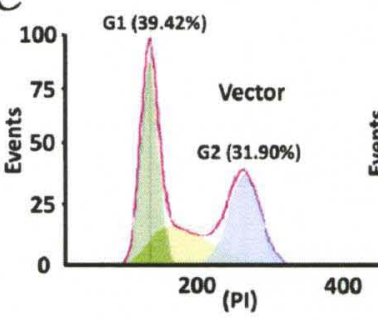

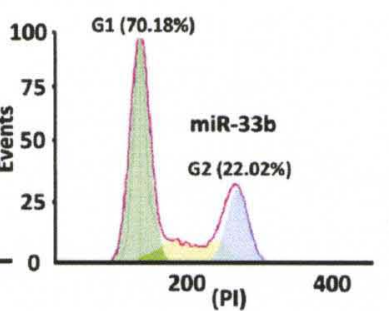
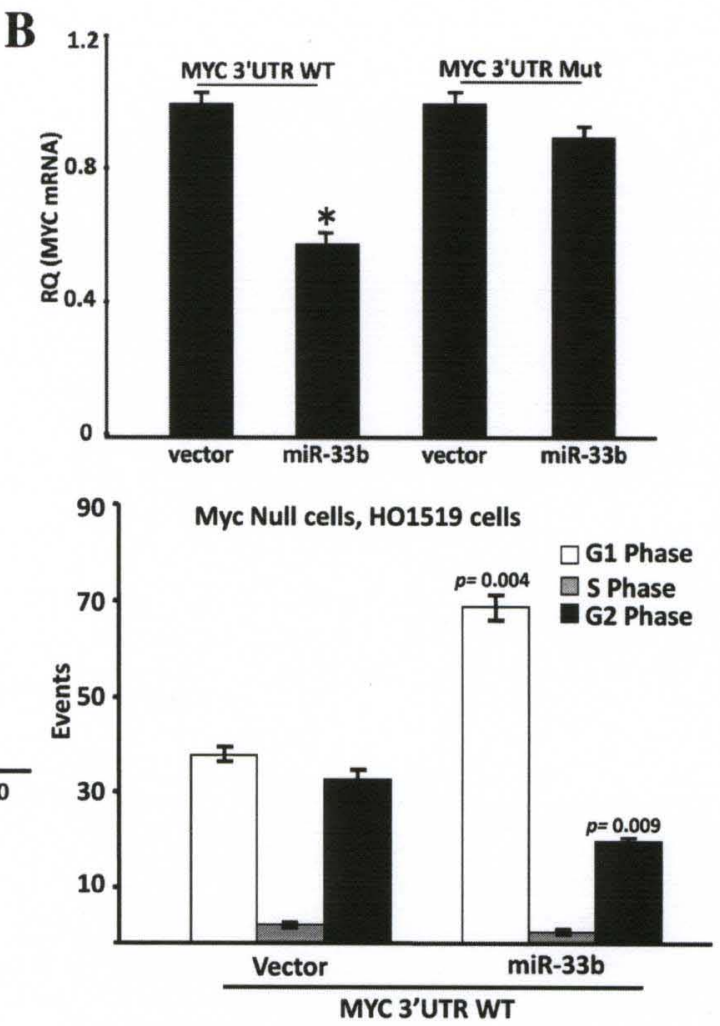

Fig. 9: miR-33b down-regulates exogenously expressed c-Myc in $M Y C$-null

HO15.19 cells. (A) Western blot analysis show that miR-33b down-regulates exogenously expressed c-Myc from a MYC construct with the 3'UTR WT, but not a 3'UTR mut, and c-Myc transcriptional target Cyclin E. (B) qRT-PCR demonstrates a reduction in $M Y C$ mRNA level following miR-33b overexpression. (C) Flow cytometry analysis for cell cycle progression of MYC-null cells following over-expression of miR-33b On the left is a representative photo of a single flow cytometry run with the y-axis donating events (number of cells) and the $\mathrm{x}$-axis donating the emitted fluorescent of the DNA dye (PI): the bar graft on the right is provided to summarize three independent runs. 


\subsubsection{Re-introduction of miR-33b in D283 cells down-regulates c-Myc and adversely affects its functions.}

The human $m i R-33 b$ gene is located in intron 17 of $S R E B F 1 \sim m i R-33 b$ gene and their genomic locus $17 \mathrm{p} 11.2$ is frequently lost in medulloblastoma. c-Myc is over-expressed in $\sim 31-64 \%$ of medulloblastoma, yet MYC amplification only accounts for $\sim 5-15 \%$ of cases (96). We hypothesize that miR-33b loss is responsible for c-Myc over expression, in medulloblastoma. We first employed D283, a medulloblastoma cell line without $17 \mathrm{p} 11.2$ with no MYC gene amplification, yet with high levels of c-Myc (203). When miR-33b was reintroduced, c-Myc expression was down-regulated, along with its transactivation targets Cyclin E and ODC, and Gadd45 $\alpha$ (a c-Myc repressed target) was up-regulated (Fig. 10A). Coinciding with down-regulation of Cyclin E, more cells arrested at the G1 phase when miR-33b was over-expressed in D283 cells (Fig. 10B). The role played by cMyc in inducing cell proliferation and apoptosis is partly due to its ability to up-regulate ODC and Gadd45 $\alpha$ expression, respectively. Over-expression of miR-33b resulted in reduction in MTT-assayed cell proliferation compared to empty vector alone (Fig. 11A). To verify whether the reduction in cell proliferation by miR-33b is dependent on MYC 3'UTR, D283 cells were transfect with a MYC-expressing construct carrying either 3'UTR WT or 3'UTR Mut, in which the miR-33b binding site was disrupted (3'UTRMut). As miR-33b targets 3'UTRWT but not 3'UTRMut, cell proliferation was reduced only in cells carrying MYC with the 3'UTRWT (Fig. 11B). We also found miR$33 \mathrm{~b}$ reintroduction down-regulated the expression of miR-9, a miRNA regulated by cMyc (204) that mediates cell migration (Fig. 11C) and resulted in a reduction in cell migration (Fig. 11D). 
Collectively, these data support that reintroduction of miR-33b into miR-33b-null D283 cells caused down-regulation of c-Myc and its transcriptional targets, decreased cell proliferation, cell cycle progression, and cell migration. 
A
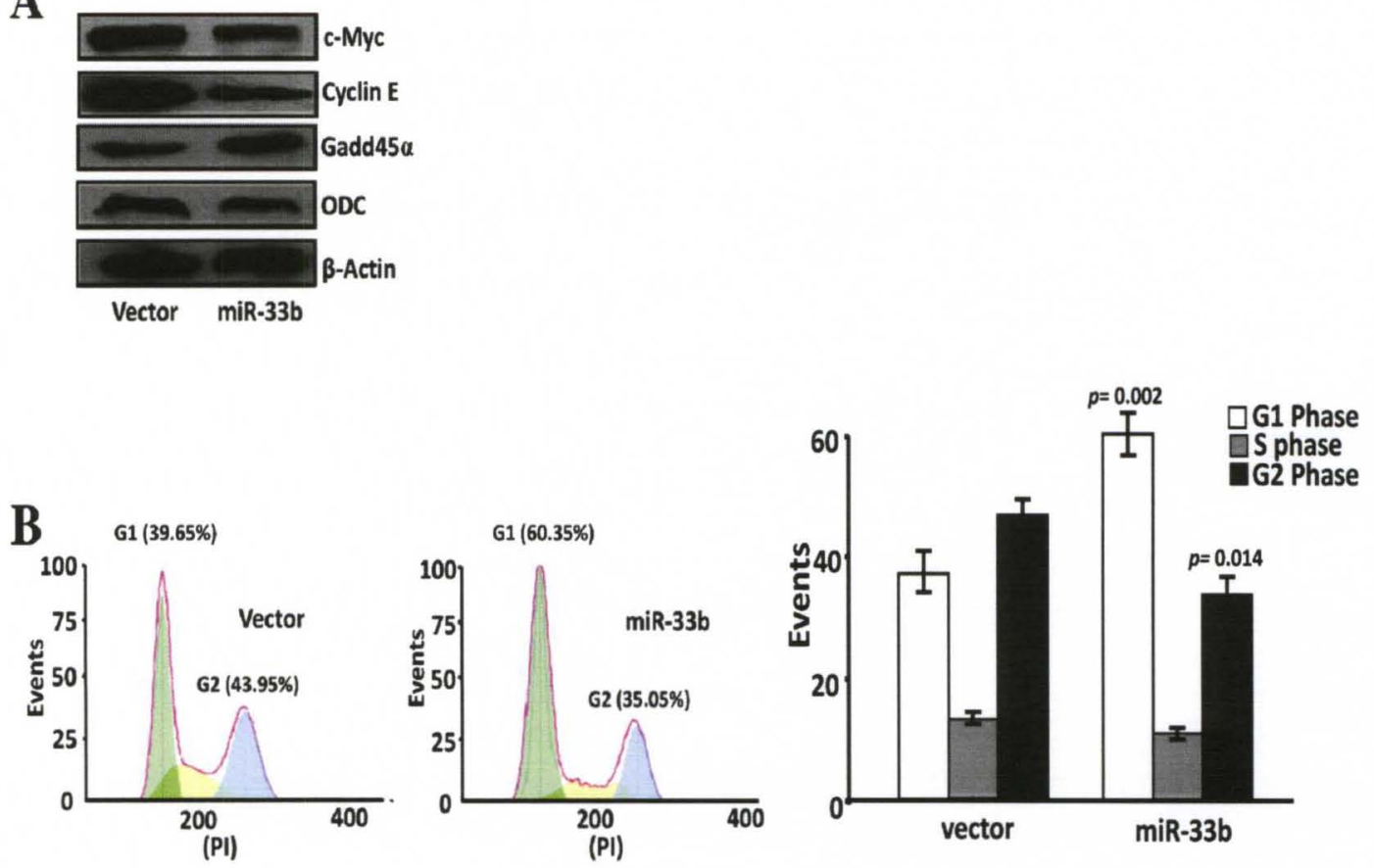

Fig. 10: Re-introduction of miR-33b in D283 cells results in negatively regulates cMyc expression and c-Myc-mediated function. (A) Western blot analysis for c-Myc, Cyclin E, ODC and Gadd45a expression in D283 cells following stable transfection with the parental vector or miR-33b. (B) Flow cytometry analysis to determine cell cycle progression of D283 cells transfected with vector or miR$33 \mathrm{~b}$. On the left is a representative photo of a single flow cytometry run with the $\mathrm{y}$-axis denoting events (the number of cells) and the x-axis denoting the emitted fluorescence of the DNA dye (PI); a bar graph on the right is provided to summarize three independent runs. 
A

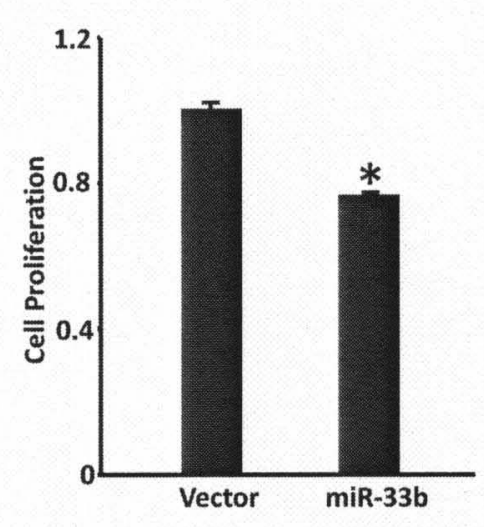

C

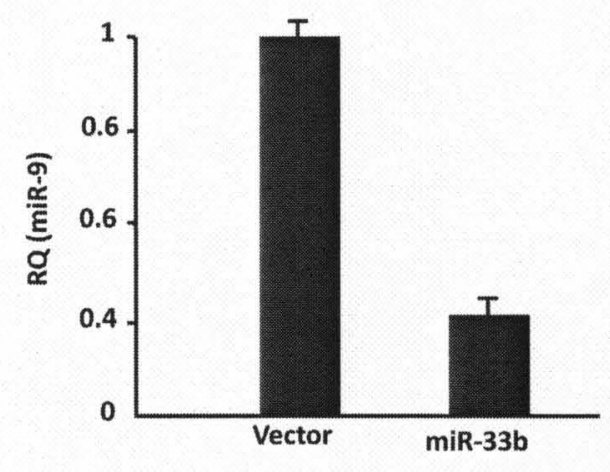

B

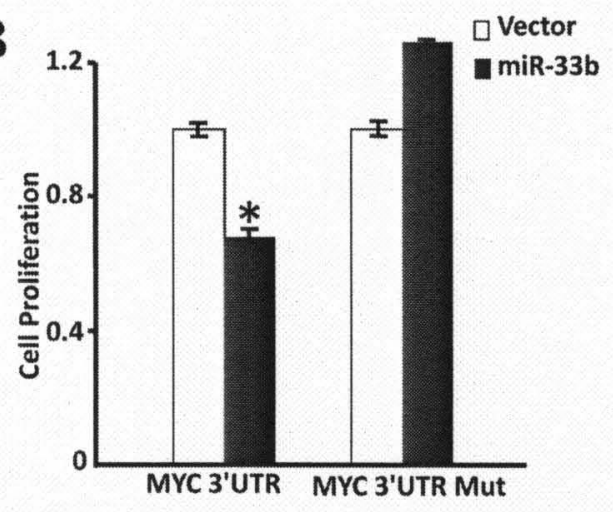

D

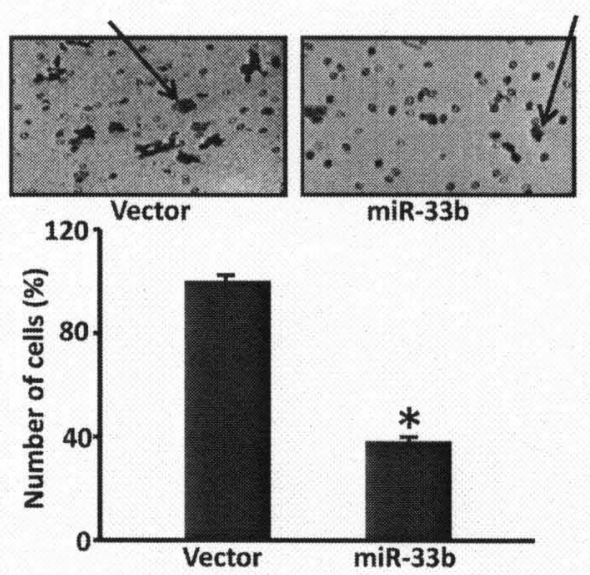

Fig. 11: miR-33b negatively affects c-Myc-mediated cell proliferation and migration of D283 cells. (A) MTT cell proliferation/viability assay of D283 cells stably transfected with vector or miR-33b. (B) D283 cell proliferation is rescued exogenous c-Myc from a MYC 3'UTR mutant construct, but not a MYC 3'UTR WT construct. (C) QRT-PCR for the expression of miR-9 in D283 cell stably transfected with vector or miR-33b. (D) Transwell cell migration assay D283 cells. The upper panel is a representative photo of migrated cells; the bottom panel is the bar graph summarizing three independent experiments. 


\subsubsection{Stem cell characteristics of $D 28$ cells are abrogated by miR-33b}

Medulloblastoma is a tumor which is characterized by an undifferentiated stem- or progenitor-like appearance in the majority of its cells with neurons, and glia cell types detected. c-Myc is a major player in the maintenance of stem cell self-renewal $(88,205)$ and appears to play a crucial role in medulloblastoma development. Medulloblastomas have been reported to have a stem cell origin and are capable of forming neurospheres when incubated in neurobasal medium $(206,207)$. Even in regular growth medium, there is a major morphological change (multicell aggregates to desegregated single cell suspension) in D283 cells stably transfected with miR-33b (Fig. 12A). When cultured in neurobasal medium, D283 cells with miR-33b formed fewer neurospheres compared to the control (Fig. 12B). In neurobasal medium, the mRNA levels of $M Y C$ and two stem cell markers SOX2 and CD133 was reduced with miR-33b overexpression, while in growth medium, miR-33b introduction resulted in decreased expression of SOX2 and $M Y C$ but not that of $C D 133$ (Fig. 12C). We also determined the expression of a neural stem cell marker Musashi, a RNA-binding protein that is essential for neurosphere formation and proliferation(208-210). miR-33b-expressing D283 neurospheres had lower levels of Musashi, while Musashi expression was not altered significantly when cells were cultured in growth medium (Fig. 12D). These results show that miR-33b expression is sufficient to activate differentiation of medulloblastoma cells via c-Myc down-regulation. Furthermore, miR-33b over-expression results in the negative regulation of stem cell characteristics of medulloblastoma cell line. 


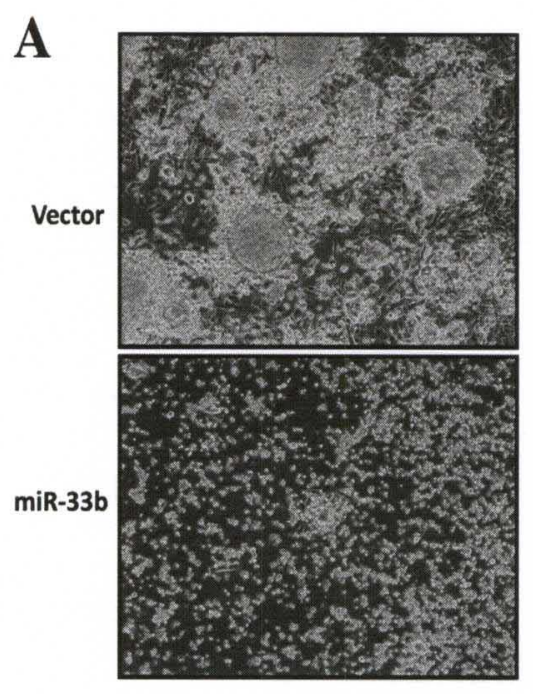

B

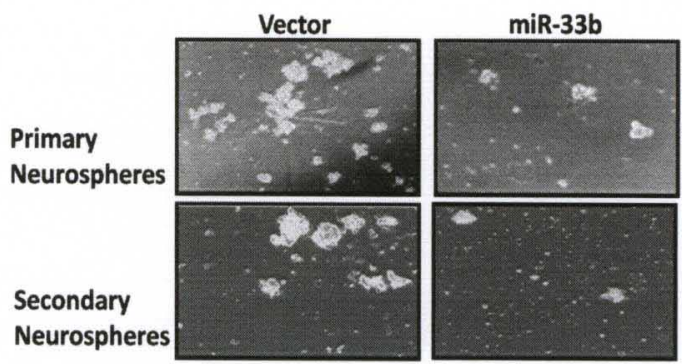

C

$\square$ Vector $\square$ miR-33b
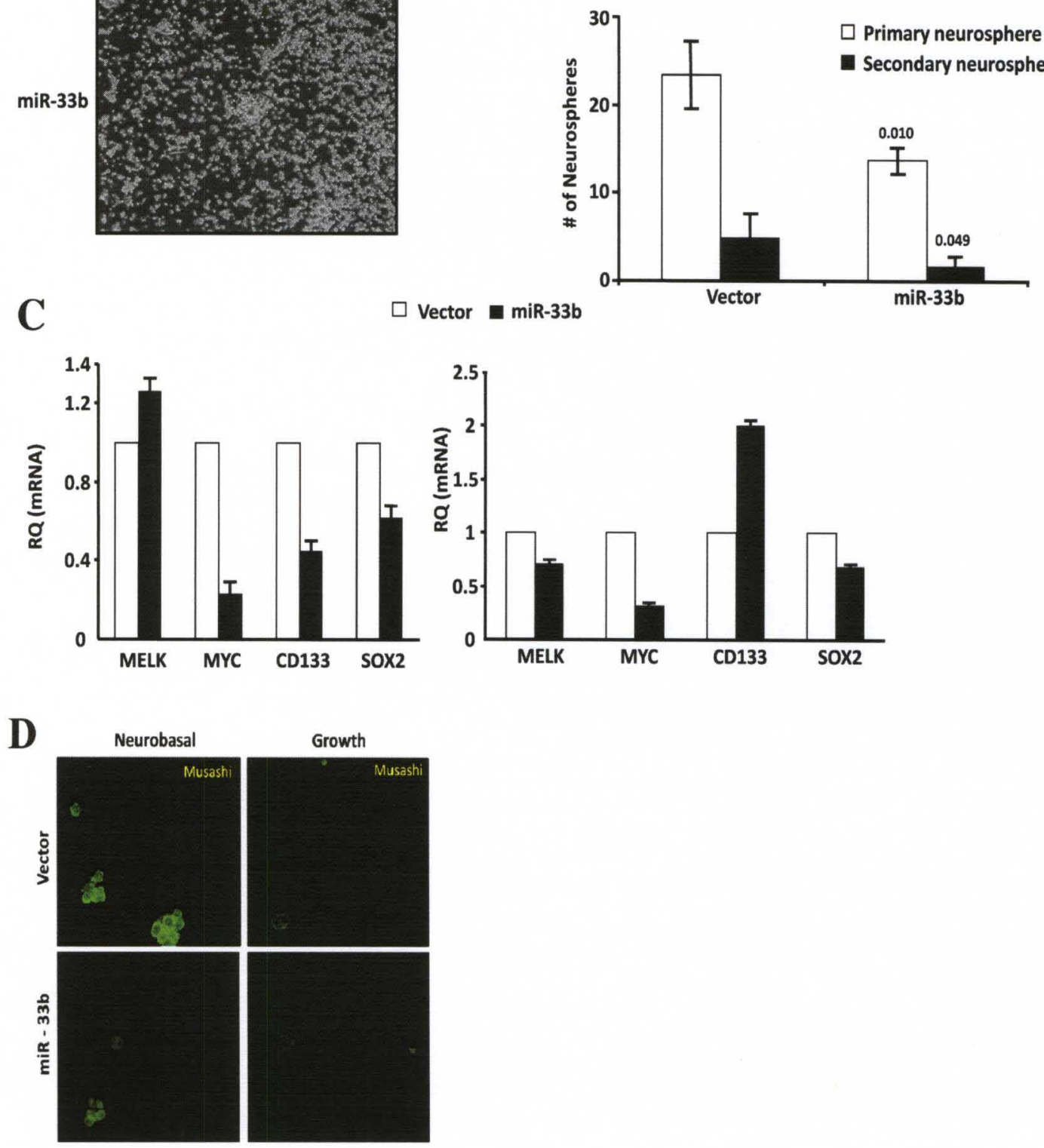
Fig. 12: Stem cell characteristics of $\mathbf{D 2 8 3}$ cells are adversely affected by miR-33b.

(A) Morphological change of D283 cells with stable expression of miR-33b in growth medium. (B) Neurosphere formation assay of D283 cells stably transfected with parental vector or miR-33b. MiR-33b is impaired in neurobasal medium. The top panel donates a representative photo of primary and secondary neurospheres; the bottom panel is a bar graph summarizing three independent experiments (C) QRT-PCR for the mRNA levels of the stem cell markers $M E L K, M Y C$, Sox2 and $C D 133$ from RNA extracted from Neurosphere or differentiated neurosphere. (D) Immunocytometry to determine the levels of musashi in stably transfected vector or miR-33b D283 neurospheres or differentiated neurospheres.MmiR-33b expression results in reduced expression of Musashi in neurospheres. 


\subsubsection{Over-expression of miR-33b in Daoy cells down-regulates c-Myc and adversely affects c-Myc-mediated cellular function}

We used a medulloblastoma cell line, Daoy, with intact SREBF1 miR-33b gene to determine whether over-expression of miR-33b will negatively affect medulloblastoma development. Daoy medulloblastoma cells are adherent cells and, unlike D283 cells, have a 'glial' phenotypic profile. Daoy are desmoblastic in origin and exhibit a less aggressive characteristic compared to the metastatically derived D283 cell line. Over-expression of miR-33b resulted in down-regulation of the protein levels of c-Myc and its targets Cyclin E and ODC (Fig. 13A). Gadd45 $\alpha$, a protein repressed by c-Myc, was up regulated (Fig. 13A). Over-expression of miR-33b in Daoy cells resulted in delay at G1cell cycle (Fig. 13B) and decreased cell proliferation (Fig. 14A). Finally, the expression of miR-9 was decreased (Fig. 14B) and cell migration was significantly reduced when miR-33b was over expressed in Daoy cells (Fig. 14C).

These results demonstrate that miR-33b has similar effects on miR-33b-negative medulloblastoma cells and miR-33b-positive medulloblastoma. Therefore miR-33b is an attractive chemotherapeutic target against medulloblastoma. 

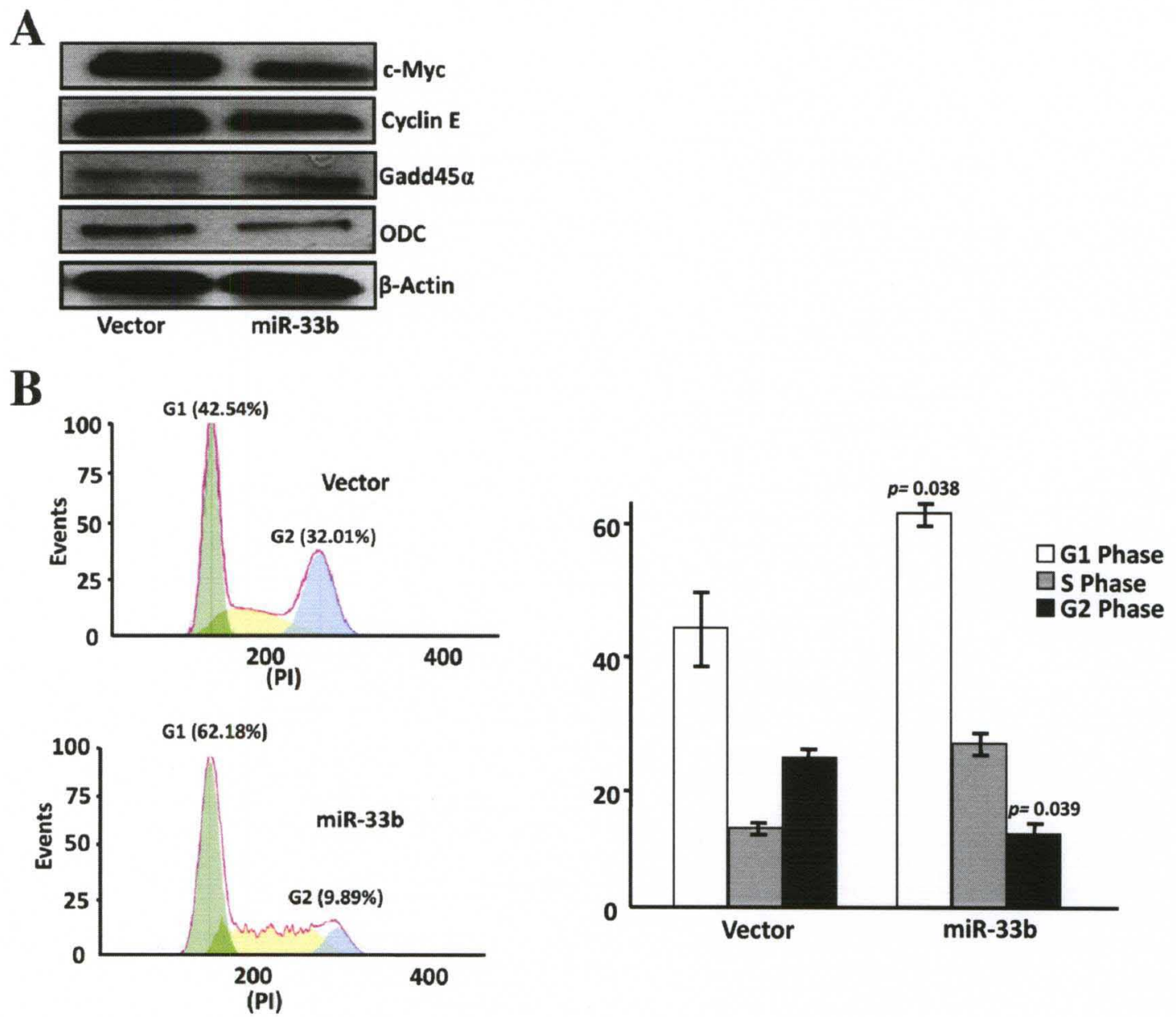

Fig. 13: Over-expression of miR-33b in Daoy cells down-regulates c-Myc and increase G1 cell cycle arrest. (A) Western blot analysis for protein levels of cMyc and its transcriptional targets Cyclin E and ODC in Daoy cells transfected with vector or miR-33b. (B) Flow cytometry analysis to determine cell cycle progression of Daoy cells transfected with the parental vector or miR-33b. On the left is a representative photo of a single flow cytometry run with the y-axis denoting events (the number of cells) and the $\mathrm{x}$-axis denoting the emitted fluorescence of the DNA dye (PI); a bar graph on the right is provided to summarize three independent runs. 

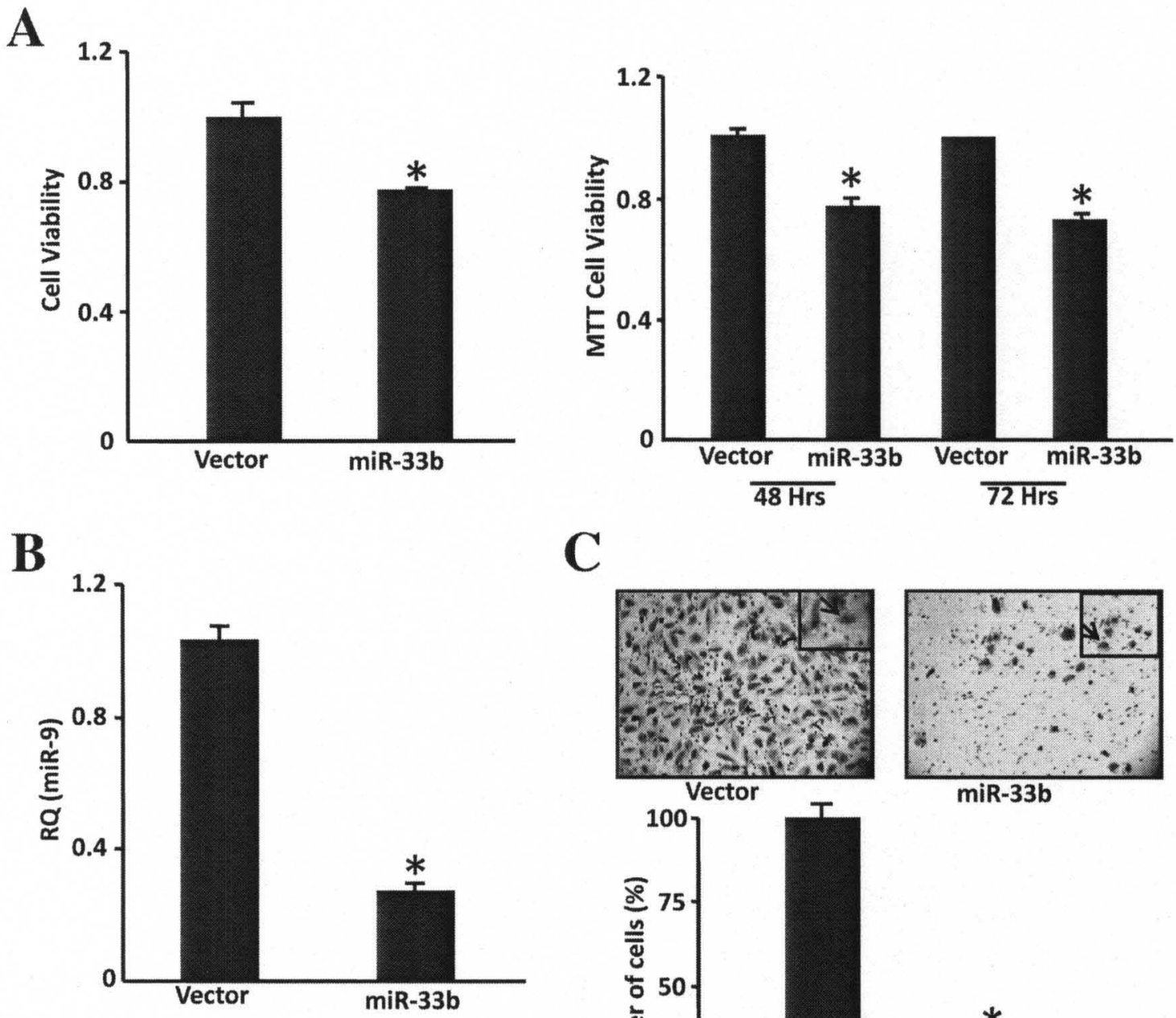

C

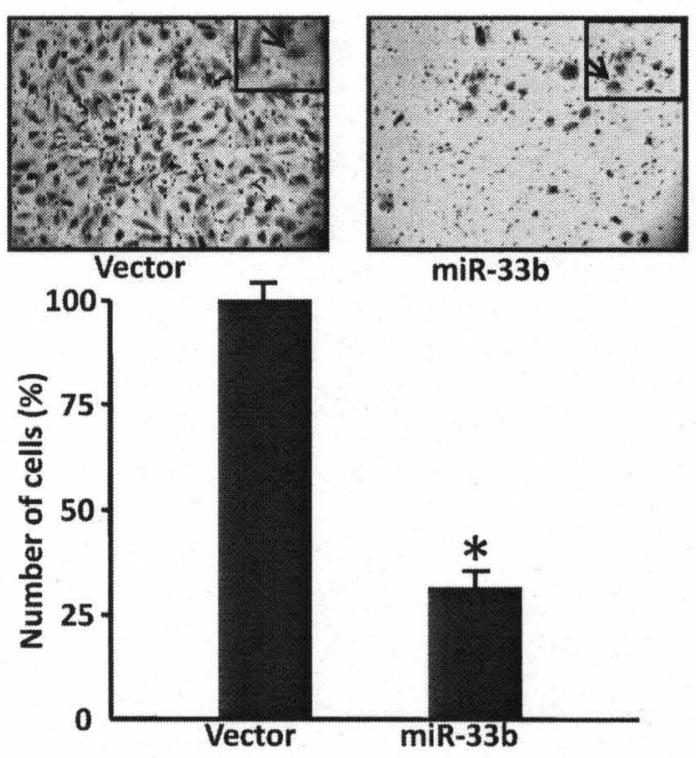

Fig. 14: miR-33b inhibits c-Myc-mediated cell proliferation and invasiveness of

Daoy cells. (A) MTT cell proliferation assay of Daoy cells transfected with parental vector or miR-33b for 24,48 or $72 \mathrm{hrs.} \mathrm{(B)} \mathrm{QRT-PCR} \mathrm{assay} \mathrm{for} \mathrm{the}$ expression of miR-9 levels in Daoy cells transfected with miR-33n or vector. (C) Daoy cells transfected with mir-33b or vector for $48 \mathrm{hrs}$ followed by Transwell migration assay. 


\subsection{8. miR-33b expression reduces tumorigenesis of D283 cells}

The soft agar colony forming ability of D283 cells was tested following stable transfection with either a vector control or miR-33b. D283 cells expressing miR-33b formed significantly less colonies on soft agar compared to D283 cells stably transfected with parental vector (Fig. 15). We then injected D283 cells constitutively expressing miR-33b or vector orthotopically into the cerebellum of immunodeficient nude (nu/nu) mice. Six weeks following injection, there was significantly less tumor expansion in brain ventricles of mice injected with cells carrying miR-33b compared to cells stably transfected with the parental vector (Fig 16A). As miR-33b is shown to down-regulate cMyc expression, we performed immunohistochemistry (IHC) on tumor sections to determine the expression level of c-Myc, Cyclin E, and sox 2 proteins. miR-33bexpressing D283 tumors expressed less c-Myc, Cyclin E and Sox 2 compared to the control (Fig. 16B). Taken together, we conclude that miR-33b decreases medulloblastoma tumor growth via c-Myc-mediated inhibition of cell cycle progression as evident by lower levels of c-Myc and Cyclin E. 


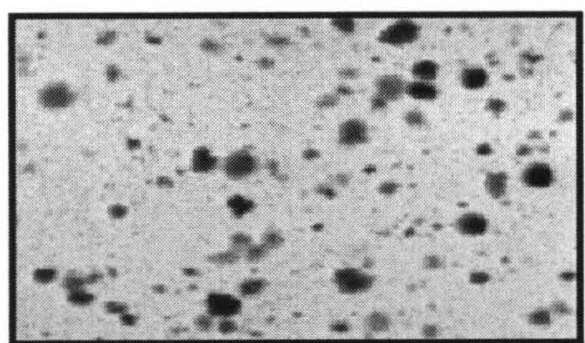

Vector

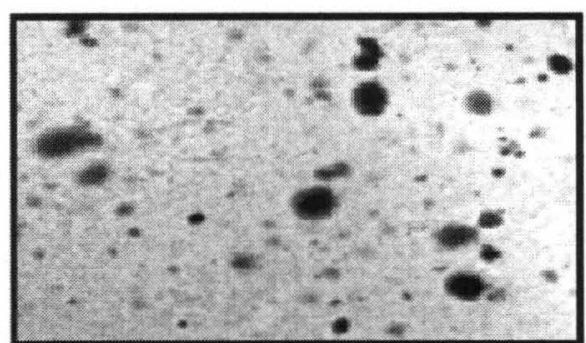

miR-33b

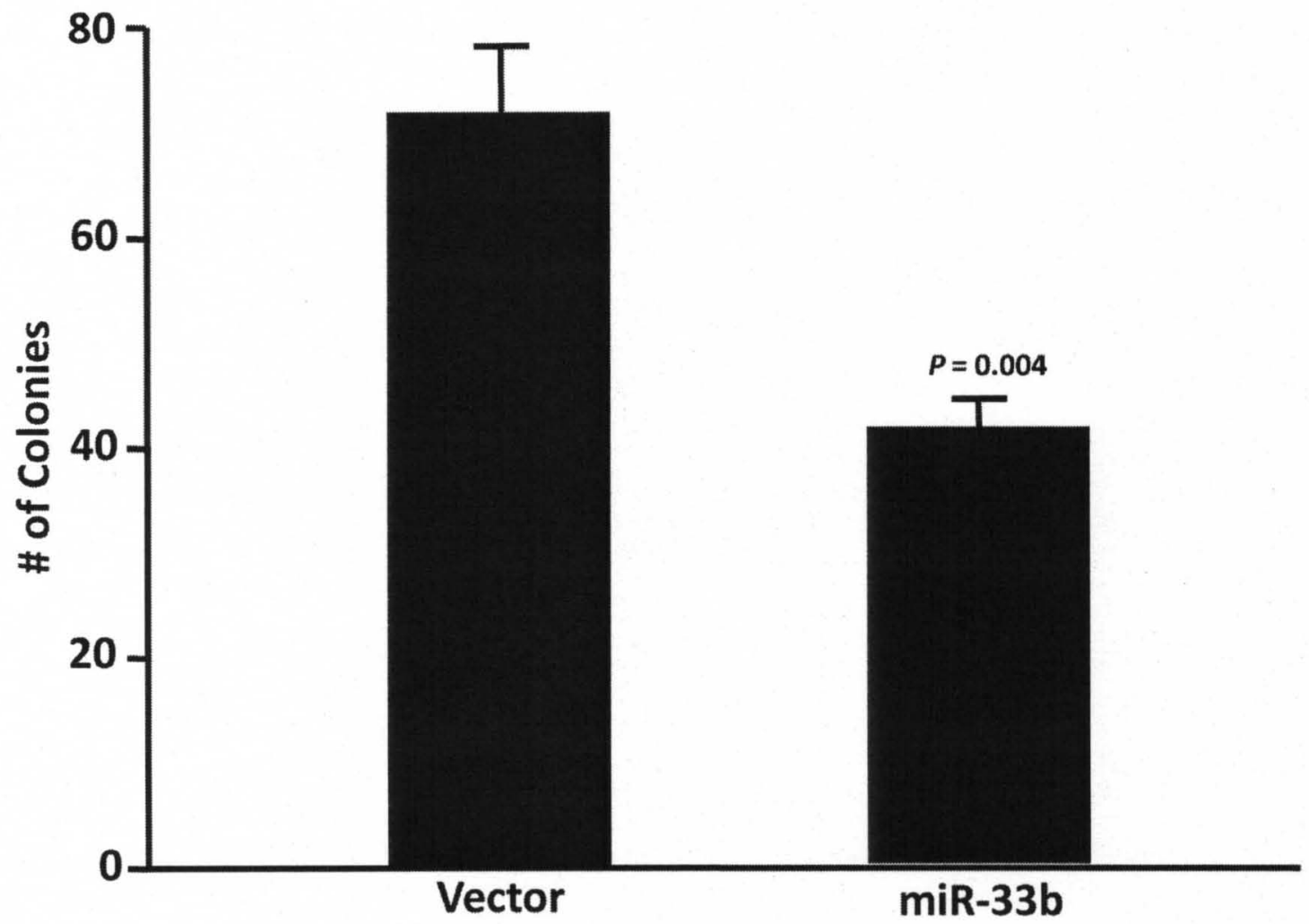

Fig. 15: miR-33b decreases anchorage-independent colony formation of D283 cells. D283 cells stably transfected with parental vector or miR-33b was grown on $0.5 \%$ softagar for two week followed by MTT staining and imaged (magnification 10X). 

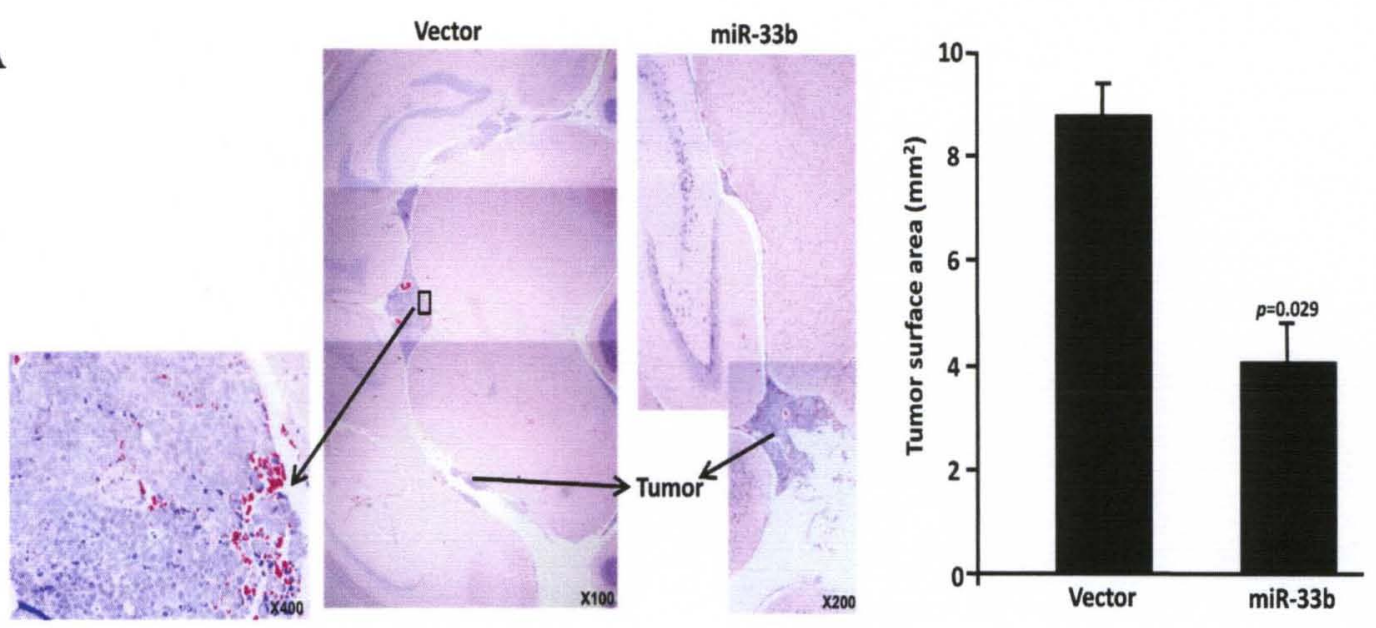

B

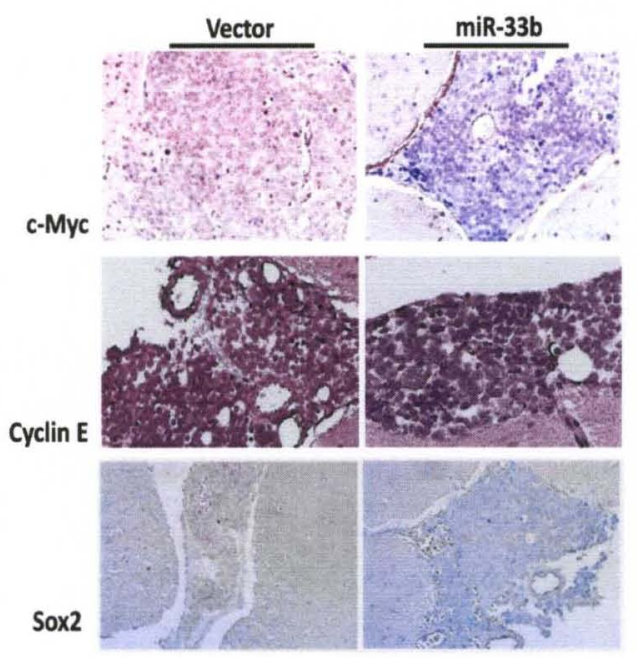

Fig. 16: miR-33b reintroduction in D283 cells reduces tumorigenicity. (A)

Hematoxylin Eosin (H\&E) staining of mouse brain injected with D283 cells stably transfected with vector or miR-33b. Tumors are light blue; red dot donates blot vessels within tumors. (B) IHC of D283 tumors from D283 cells stably transfected with the parental vector or miR-33b for the expression of cMyc, Cyclin E and Sox2. Protein expression range is from brownish (high expression) to light blue (no expression). 


\subsection{DISCUSSION}

$M Y C$ codes for $\mathrm{c}-\mathrm{Myc}$, a transcription factor that regulates the expression of up to one third of human genes $(185,186)$. The genes regulated by c-Myc have placed it at the center of many biological processes, including apoptosis, cell cycle, differentiation and cellular proliferation $(96,211-215)$. It is now well established that c-Myc expression is essential for stem cell biology and inhibits differentiation of cells while favoring proliferation. The importance of c-Myc in carcinogenesis is also supported in animal models (216). c-Myc is viewed as an attractive target against cancer due to its crucial role in tumorigenesis. Despite early promise shown by drugs developed to target c-Myc, complete success is hampered by the lack of a good compound that inhibits c-Myc and low specificity of current chemotherapeutics against $M Y C$. Therefore, understanding how Myc is regulated at all levels is critical for a more specific targeted therapy to be developed. The major goals of this work aimed at studying and identifying miRNAs that target MYC and how these miRNAs contribute to cancer development. We used a 2-tier luciferase screening method to systematically identify miRNAs that target the MYC gene. In tier-1, we made use of the c-Myc binding E2F2 promoter upstream of a luciferase gene. This enabled us to evaluate miRNAs that affected the MYC pathway. In tier-2, we cloned the 3'UTR of MYC downstream of a Rluc gene to evaluate which miRNAs directly target the 3'UTR of MYC. Using these E2F2-luc and MYC 3'UTRbased Rluc constructs we screened 400 and 54 miRNAs, respectively, and showed that more than $25 \%$ of the miRNAs in each group adversely regulated MYC. Our luciferase results suggest that various miRNAs affect MYC directly as well as other components of 
the MYC pathway. This result serves as a first step in elucidating miRNA targets in the MYC pathway.

Using miR-210 as a control, we confirmed that our luciferase data are reliable since miR210 up-regulated the reporter assay from the E2F2-luc construct, consistent with a previous study showing that miR-210 targeted a c-Myc antagonist, MNT, to activate cMyc (217). Also, Let-7a and the miR-17 92 cluster of miRNAs target the MYC gene as reported previously $(218,219)$ and this is consistent with our luciferase data which showed that let-7a and members of the miR-17 92 cluster down-regulate the luciferase activity of both the E2F2-Luc and MYC 3'UTR based constructs. Our luciferase data suggest that miR-33b and miR-203 negatively regulated the luciferase reporter from the E2F2 promoter and the $3^{\prime} U T R$ based constructs. However, only miR-33b over-expression resulted in down-regulation of c-Myc protein levels and this corresponded with decreased levels of c-Myc targets, Cyclin E and ODC. Also, we demonstrated an up regulation of Gadd45 $\alpha$, a gene that is repressed by c-Myc. The mRNA-miRNA binding usually results in either translational repression or mRNA degradation. Translational repression was believed to be the dominant mechanism observed for the majority of human miRNAs since perfect complementarity is hardly encountered (220). This observation are particularly true for mRNAs that have multiple binding sites to a given miRNA (220). However, when the miRNA has just one binding site to the mRNA's 3'UTR, speedy mRNA degradation is favored (220). We used qRT-PCR assay to reveal that miR-33b over-expression leads to lower steady state levels of MYC mRNA, favoring a mRNA degradation mechanism for this pair. 
Other mechanisms likely contribute to c-Myc over-expression in medulloblastoma, as cMyc is over-expressed in $\sim 31-64 \%$ of cases (221-226), but the MYC gene is amplified in only $5-15 \%(94,99,100)$. We show that miR-33b, a miRNA whose gene locus on chromosome $17 \mathrm{p} 11.2$ is characterized to be a major breakpoint in a subset $(\sim 17 \%)$ of medulloblastoma, regulates c-Myc expression and is sufficient to decrease medulloblastoma development via reduced cell proliferation, increased G1 cell cycle arrest and decrease cell migration. Similar experiments were performed on a desmoplastic medulloblastoma cell line, Daoy, with similar results. Unlike the anaplastic D283 medulloblastoma cells which are characterized by loss of chromosome 17p11.2 and over-expression of c-Myc $(227,228)$, the desmoplastic Daoy cells have an intact chromosome $17 \mathrm{p} 11.2$ locus and express miR-33b. This suggests that loss of miR-33b expression is a contributing factor to aggressiveness of tumor cell growth. Our data show that expression of miR-33b decreases tumorigenic properties such as proliferation, cell migration and cell cycle progression. Colony formation assays on soft agar show that miR-33b was able to reduce the number of D283 colonies compared to empty vector control.

Medulloblastoma has been reported to have a stem cell origin (229). Medulloblastoma cells differentiate into numerous cell types, lending credence to its stem-like character. Furthermore, a group of cancer cells with the ability to self-renew and differentiate into all cell types continue to gain ground as the major culprit in tumor therapeutic resistance $(230,231)$. Development of therapies that could result in targeting the differentiated and the self-renewing cancer cells will significantly reduce relapses. We used D283 cells to 
determine whether miR-33b expression will affect the stem cell characteristics of medulloblastoma. We show that stable re-introduction of miR-33b resulted in a morphological change in the D283 cells, from a multicell aggregate characteristic of stem cell to desegregated single-cell suspension characteristic of differentiated cells. miR-33bexpressing D283 cells when placed in the neurobasal media that favors the growth of neural stem cells, formed smaller and lower number of neurospheres compared to the control. Expression of stem cell markers, MYC, SOX2, CD133, and Musashi was significantly reduced in miR-33b-expressing neurospheres and differentiated neurospheres compared to cells stably transfected with the parental vector. These results suggest that miR-33b targets both the differentiated cancer cells and the self renewing cells. To replicate our findings in vivo, D283 cells with stable expression of miR-33b or parental vector using lentivirus infection was injected into the cerebellum of immunodeficient nude mice to determine whether tumorigenesis is affected. miR-33bexpressing D283 cells formed smaller tumors compared to D283 tumors from cells stably transfected with the parental vector. Expression levels of c-Myc, Cyclin E and Sox2 was down-regulated from D283 tumors expressing miR-33b. Differential expression of Cyclin E indicated that miR-33b acted as a cell cycle regulatory miRNA and functions through a c-Myc-mediated arrest in cell growth.

In summary, data from these experiments suggest that miR-33b is a negative regulator of c-Myc, and its loss in a subset of medulloblastomas may contribute to c-Myc over production and tumor growth. 


\section{CHAPTER 3}

\section{A STATIN-REGULATED MICRORNA REPRESSES $C$-MYC EXPRESSION AND FUNCTION}

\subsection{INTRODUCTION}

RNA interference (siRNA) technology show great promise based on studies in mammalian cell-culture systems and animal in vivo models and is currently being extensively evaluated as a potential therapeutic strategy. The induction of immune response by siRNA (232) is a drawback of siRNA technology, as well as an observed increase in mortality, probably due to saturation of the miRNA-processing system. However, the valuable experience and the knowledge gained from siRNA-based therapy are used while design miRNA-based technologies. The rationale for using miRNAs as potential therapeutic targets is based on: (1) miRNAs are endogenous antisense regulatory molecules; (2) miRNA expression profiles can be used to diagnose disease states, and deregulated miRNAs contribute to the initiation and development of disease; (3) mouse models demonstrate that miRNAs play an important role in diseases; (4) miRNA expression levels respond to physiological stimuli; (5) miRNA expression profiles change when cells are treated with drugs in vitro; and (6) their small size (23-24 nucleotides in length) makes them attractive for drug development. miRNAs are related to and function similarly to siRNA $(233,234)$, which was first identified by Fire et al. (235). Various miRNA-based therapies including anti-sense miRNAs (236), locked nucleic acid (234), antagomirs (237), virus vector expressing miRNA (232) and mimic 
miRNAs (156) have been examined, with limited success. Many difficulties are encountered when considering miRNAs as therapeutic drugs including (1) the extensive number of targets one miRNA can regulate, (2) delivery to the disease site; (3) lack of tissue specificity; (4) the potential of the body eliciting an immune response against them; and (5) low stability in vivo since they may be degraded by endogenous RNases. Improving the stability of miRNA-based drugs is the major focus of drug design. Furthermore, to develop effective delivery systems, it is important to gain an in-depth understanding of cellular miRNA uptake, distribution and the biological activity at whole-body, organ, and cellular levels. To circumvent these difficulties, repurposing FDA-approved drugs is a more realistic proposition. Various studies have indicated differential miRNA expression profiles upon treatment of cells with various compounds $(238,239)$. For example, breast cancer cells resistant to doxorubicin (DOX) exhibit a pronounced deregulation of miRNA expression and altered expression of miRNA processing enzymes (238). It is therefore possible to modulate specific miRNA production with approved FDA compounds. However, two drawbacks must be considered: (1) validation of positive proof-of-concept clinical data for the repurposed compounds; (2) the ability of the drug to be delivered to the diseased site.

Drug repurposing (also known as Drug re-profiling, Therapeutic Switching and Drug retasking) refers to the development of existing drugs for new indications. These drugs may have (i) known therapeutic uses, (ii) failed to show efficacy in late stage clinical trials, without safety issues; (iii) stalled in development for commercial reasons; (iv) passed the point of patent expiry; or (v) are being explored in new geographical markets (240). 
Using drug repositioning, pharmaceutical companies have achieved a number of successes, for example Pfizer's Viagra in erectile dysfunction and Celgene's thelidomide in severe erythema nodosum leprosum (240). We hypothesize that drug repurposing via modulation of miR-33b expression will decrease proliferation of miR-33b-positive medulloblastoma cells.

We used a miR-33b-positive medulloblastoma cell line to screen drugs in the NIH collection that up-regulated miR-33b and reduced cell proliferation, and specifically singled out lovastatin. We showed that lovastatin results in a reduction in cell proliferation of miR-33b-positive Daoy cells, an effect not significant in miR-33bnegative D283 cells. The lovastatin-induced inhibition of Daoy cell proliferation correlated with an increase in miR-33b expression as well as a down-regulation of c-Myc expression. Also, Daoy tumor growth was significantly reduced in mice treated with lovastatin, and tumors expressed lower levels of miR-9, c-Myc and Cyclin E. Treatment with lovastatin and the methylation inhibitor 5-Azacytidine, which released methylation of the SREBF1 miR-33b promoter, increased sensitivity to lovastatin in HeLa cells, a cell line that showed reduced lovastatin sensitivity, via miR-33b up-regulation.

\subsection{MATERIALS AND METHODS}

\subsubsection{Cell culture}

Medulloblastoma cells, D283 and DAOY, were purchased from ATCC and cultured in EMEM (Gibco, Carlsbad, CA) supplemented with 10\% FBS and $0.1 \%(\mathrm{v} / \mathrm{v})$ AntibioticAntimycotic (Cellgro, Manassas, VA). UW228 medulloblastoma cell line was the kind 
gift of Dr. J.R. Silber (University of Washington, Seattle, WA) and were maintained in DMEM/F12 supplemented with $10 \%$ FBS and $0.1 \%$ (v/v) antibiotic-Antimycotic (Cellgro, Manassas, VA). The human cervix carcinoma cell line (HeLa cells) was

purchased from ATCC and cultured in DMEM (Gibco, Carlsbad, CA) supplemented with 10\% FBS and $0.1 \%(\mathrm{v} / \mathrm{v})$ Antibiotic-Antimycotic (Cellgro, Manassas, VA). All cell lines were cultured at $37^{\circ} \mathrm{C}$ in an atmosphere containing $5 \% \mathrm{CO}_{2}$. Transient and stable transfections were performed as previously described.

\subsubsection{Luciferase assay}

The LightSwitch Luciferase Assay Kit (SwitchGear, Menlo Park, CA) was used to determine whether lovastatin activates the $S R E B F 1$ promoter. The $S R E B F 1$ promoter was cloned upstream of a Rluc gene. Daoy and D283-Med cells in 96-well plates were transfected with the SREBF1-luc promoter construct (SwitchGear Genomics, Menlo Park, CA), an LDHA-PROM positive control (SwitchGear, Menlo Park, CA) and a negative control R01-PROM (SwitchGear, Menlo Park, CA) in triplicate wells. The cells were treated $24 \mathrm{hrs}$ following transfection with $0,0.2,2$ or $10 \mu \mathrm{M}$ of lovastatin. Luciferase activity was measured 24 hrs later, using the LightSwitch Luciferase Assay Kit, per the manufacturer's instruction. Briefly, $100 \mu 1$ of the assay solution, made-up of $1 \mathrm{x}$ substrate in assay buffer, was pippeted into each well and incubated in the dark for 30 mins, followed by luciferase reporter measurement. Luciferase activity was normalized against values of the positive and negative control.

\subsubsection{Western blot analysis}


Medulloblastoma cell lines D283, Daoy and UW228 $\left(1 \times 10^{6}\right.$ cells/well) cultured in 6well plates were treated with lovastatin $(0,0.2,2,10 \mu \mathrm{M})$ and total protein was extracted using 1X RIPA reagent (Cell Signaling Technology, Danvers, MA) containing 1X protease inhibitor cocktail (Sigma, St. Louis, MO) after $48 \mathrm{hrs.} \mathrm{Total} \mathrm{protein}$ concentration was determined using the Bicinchoninic Acid (BCA) method (Thermo scientific, Rockford, IL) according to the manufacturer's protocol. Western blot for cMyc (Santa Cruz, Santa Cruz, CA; sc-40; diluted 1:1000), Cyclin E (Santa Cruz, Santa Cruz, CA; sc-25303; diluted 1:1000), Gadd45 $\alpha$ (Santa Cruz, Santa Cruz, CA; sc-6850; diluted 1:1000), ODC (Cell signaling; Danvers, MA; dilution 1:1000) and $\beta$-Actin (Sigma, St. Louis, MO; AC-15; diluted 1:5000) expression was performed. HeLa cells in 6 well plates were treated with lovastatin $(0,0.2,2,10 \mu \mathrm{M})$ or lovastatin $(0.2 \mu \mathrm{M}), 5$ Azacytidine $(0.2 \mu \mathrm{M})$ or lovastatin $(0.2 \mu \mathrm{M}) / 5$-azacytidine $(0.2 \mu \mathrm{M})$ for $48 \mathrm{hrs}$. Total protein from treated cells was used for western blot analysis for c-Myc, Cyclin E, ODC,

Gadd $45 \alpha$ and $\beta$-Actin expression. To determine whether exogenous miR-333b regulated c-Myc protein expression, HeLa cells $\left(1 \times 10^{6}\right.$ cells/well $)$ cultured in 6-well plates were transfected with $2.5 \mu \mathrm{g}$ of pSIF, miR-33b, or miR-33bM. Total protein was used for western blot analysis for the expression of c-Myc, Cyclin E, Gadd45 $\alpha$, ODC, and $\beta$ Actin.

\subsubsection{Quantitative real-time PCR (qRT-PCR)}

We used TaqMan miRNA and gene expression assays (applied Biosystem inc., Forest City, CA) to detect the expression level of mature miRNAs and gene mRNA, respectively (198). U6 RNA and $\beta$-Actin mRNA was used for normalization. 
Medulloblastoma D283, Daoy and UW228 cells were treated with lovastatin $(0,0.2,2$, or $10 \mu \mathrm{M})$ and TaqMan qRT-PCR was performed for the expression of miR-33b, MYC mRNA and SREBF1 mRNA. We performed qRT-PCR using total RNA from Daoy cells treated with vehicle, lovastatin $(0.2 \mu \mathrm{M})$, mevalonate $(100 \mu \mathrm{M})$ or lovastatin/mevalonate to determine whether mevalonate inhibits lovastatin-induced up-regulation of miR-33b and SREBF1 mRNA and down-regulation of MYC mRNA. For this, Daoy cells will be treated with vehicle, lovastatin, mevalonate and lovastatin/mevalonate for $48 \mathrm{hrs.} \mathrm{Total}$ RNA was then extracted using TriZol and TaqMan miRNA and gene expression assay was used to measure miR-33b/a, SREBF and $M Y C$ mRNA expression. To determine whether orthotopic brains tumors of Daoy and D283 treated with vehicle or lovastatin had differential levels of miR-33b and miR-9, total RNA was extracted using the TriZol reagent (Invitrogen, Carlsbad, CA) as per the manufacturer's protocol. A total of 100ng of total RNA was used for TaqMan miRNA expression assay to measure the expression level of miR-33b and miR-9. To determine the effect of lovastatin on miR-33b expression, HeLa cells were treated with lovastatin $(0,0.2,5$ and $10 \mu \mathrm{M})$ and qRT-PCR was performed 24 and $48 \mathrm{hrs} \mathrm{later} \mathrm{for} \mathrm{miR-33b} \mathrm{and} \mathrm{MYC} \mathrm{mRNA} \mathrm{expression.}$ Furthermore, HeLa cells were treated with lovastatin $(0.2 \mu \mathrm{M})$, Valproic acid $(0.2 \mu \mathrm{M}), 5$ Azacytidine $(0.2 \mu \mathrm{M})$, lovastatin/valproic acid or lovastatin/5-azacytidine for $48 \mathrm{hrs}$. Total RNA was extracted from treated cells and qRT-PCR performed for MYC mRNA and miR-33b expression. To determine whether exogenous miR-33b regulated the expression of c-Myc-mediated miR-9, HeLa cells were transfected with: empty vector or miR-33b; miRNA inhibitor negative control or anitisense-miR-33b. Total RNA was extracted from 
these cells after $48 \mathrm{hrs}$ and miR-9 levels were determined using the miRNA Taqman realtime PCR method as previously described

\subsubsection{MTT assay}

Daoy cells ( $3 \times 10^{4}$ cells/well) were plated in 96 -well plates and treated with $0.2 \mu \mathrm{M}$ of drugs in the NIH clinical collection (NCC) 1 and 2 in triplicate wells. MTT assay was performed after 48 hrs. Likewise, Daoy and D283 cells were treated in triplicate 96-well plate with lovastatin $(0,0.2,2,10 \mu \mathrm{M})$ for $48 \mathrm{hrs}$ followed by MTT assay. HeLa cells ( 3 X $10^{4}$ Cells/ well) were treated with lovastatin $(0,0.2,2$ and $10 \mu \mathrm{M})$ for $48 \mathrm{hrs}$ followed by MTT assay. To determine whether epigenetic regulation affects HeLa cells lovastatin sensitivity, HeLa cells ( $3 \times 10^{4}$ Cells/ well) in a 96-well plate were treated with vehicle or lovastatin $(0.2 \mu \mathrm{M})$ alone or with valproic acid $(0.2 \mu \mathrm{M})$ or 5 -azacytidine $(0.2 \mu \mathrm{M})$ for $48 \mathrm{hrs}$. Cell viability of treated cells was compared to normalized vehicle control. HeLa cells $\left(3 \times 10^{4}\right.$ cells/well) in 96-well plates were transfected with $0.1 \mu \mathrm{g}$ of $\mathrm{pSIF}$, miR-33b in triplicate wells. MTT assay was performed at 2, 4 and 6 days post-transfection as previously described. Furthermore, HeLa cells were co-transfected with vector control or miR-33b and pcDNA3.1, pcDNA-MYC-MYC 3'UTR WT or pcDNA-MYC-MYC 3'UTR mut. MTT assay was performed $48 \mathrm{hrs}$ later to determine if exogenous c-Myc could rescue the miR-33b-induced decrease in cell proliferation.

\subsubsection{Apoptosis assay}

To determine whether exogenous over-expression of miR-33b increase apoptosis of HeLa cells, HeLa cells ( $1 \times 10^{4}$ cells/well) in 12-well plates were transfected with $1 \mu \mathrm{g}$ of empty 
vector or miR-33b. Equally, HeLa cells were co-transfected with vector or miR-33b and pcDNA MYC, pcDNA-MYC 3'UTR WT or mutant constructs in 12 well plates. Transfected cells were washed with $1 \mathrm{X}$ PBS and suspended in $500 \mu \mathrm{l}$ of fresh media at 48,72 and 144 hrs post transfection. PI $(1 \mu \mathrm{g} / \mathrm{ml})$ was added and cells incubated in the dark for 15 mins. Cells were analyzed by flow cytometry (Becton Dickinson, Franklin Lakes, $\mathrm{NJ}$ ) within $1 \mathrm{hr}$ of staining.

\subsubsection{Cell cycle analysis}

Also, D283 and Daoy cells were treated with lovastatin $(0,0.2,2$, or $10 \mu \mathrm{M})$. The cells were collected 48 hrs later and flow cytometry was performed and cell cycle analyzed using FlowJo software (Tree Star Inc., Ashland, OR) as previously described. Likewise HeLa cells were treated with lovastatin $(0,0.2,2,10$ or $40 \mu \mathrm{M})$ or $0.2 \mu \mathrm{M}$ of lovastatin, 5-azacytidine, or lovastatin/5-azacytidine for $48 \mathrm{hrs}$ followed by cell cycle analysis. To determine the effect on cell cycle after over-expression of miR-33b, HeLa cells $\left(1 \times 10^{4}\right.$ cells/well $)$ in 12 -well plates were transfected with $1 \mu \mathrm{g} /$ well $\mathrm{pSIF}, \mathrm{miR}-33 \mathrm{~b}$ or miR-33a. For each variable a minimum of 3 samples were quantified.

\subsubsection{Transwell cell migration assay}

Daoy and D283 cells were treated with $0.2 \mu \mathrm{M}$ of lovastatin for $48 \mathrm{hrs}$. The cells were washed twice in $1 \mathrm{X}$ PBS after $48 \mathrm{hrs}$ and collected in serum-free EMEM media (Gibco, Carlsbad, CA). Transwell (Boyden) cell migration assay was then performed as previously described. Migrated cells were fixed with methanol for 10 mins and stained with $0.4 \%$ crystal violet for $2 \mathrm{hrs}$. Non-migrated cells on the upper side of the filter were 
removed with a cotton swab, and the filters mounted on microscope glass slides. Images were captured using a Nikon Eclipse TE300 microscope and a Nikon Plan Fluor 10x0.30 objective. The migrated cells were counted using the ImageJ software. To determine the effect of exogenous miR-33b on HeLa cells migration, HeLa cells $\left(1 \times 10^{4}\right.$ cells/well) were transfected with empty vector, miR-33b, miRNA inhibitor negative control or antisense miR-33b. Two days after transfection the HeLa cells were collected in fresh serum-free DMEM and Transwell assay performed.

\subsubsection{Tumor formation}

All animal experiments were conducted according to a protocol approved by the University of Louisville Animal Care and Use Committee (IACUC number: 08107). We used orthotopically medulloblastoma cell-induced mice tumor to determine whether our In Vitro data is replicable in an animal model. Daoy cells $\left(5 \times 10^{6}\right.$ cells/mice $)$ were injected subcutaneously into 13 NCRNU-M nude mice. Briefly, the cells were trypsinized and washed twice in 1X PBS. The cells were then resuspended in 1X PBS containing 5\% matrigel. The cells were then counted using the Countess meter (Invitrogen, Carlsbad, CA). A total of $5 \times 10^{6}$ cells in $400 \mu 1$ of media were injected into each mouse ( $200 \mu \mathrm{l}$ at each flank of the mice). The animals were monitored for the formation of tumors. Once tumor reached a volume of $200 \mu \mathrm{m}$, the mice were separated into 3 groups ( 4 animals per treatment group) and the mice were treated three times a week with $0,0.2$ or $1 \mathrm{mg} / \mathrm{Kg}$ by peritoneal injection. The tumor sizes were measured each week and volume calculated using the formula; Volume $=(\pi / 6)($ length $)$ (width) (height) for four weeks after which the mice were sacrificed, tumor sizes measured and tumors 
removed. Also, miR-33b-positive medulloblastoma cell line Daoy and miR-33b-negative medulloblastoma cell line D283 ( $1 \times 10^{5}$ cell/mice) were injected orthotopically into the cerebellum of the 22 mice/cell line as previously described. The mice were housed and monitored for two weeks for any signs of distress. Two weeks after orthotopic injection, the mice were treated with vehicle ( 5 mice) or $1 \mathrm{mg} / \mathrm{kg}$ of lovastatin ( 5 mice) per cell line by intraperitoneal injection. The mice were sacrificed after 4 weeks of lovastatin treatment and tumor weight measured relative to whole brain. The tumors were then incubated in $10 \%$ formalin for 3 days, embedded in paraffin and $5 \mu \mathrm{M}$ sections cut onto slides. H\&E staining was performed to determine size of D283tumors from mice treated and Daoy tumors as previously described. Immunohistochemistry (IHC) was performed using sections from the tumor groups to determine expression level of c-Myc, Sox2, Cyclin E, PCNA and Caspase 7 as previously described.

\subsubsection{EpiTect methy qPCR assay}

The EpiTect methyl DNA restriction Kit (Qiagen, Frederick, MD) and the EpiTect Methyl qPCR Primer Assay for SREBF1 (Qiagen, Frederick, MD) were used to determine the methylation status of the SREBF1 promoter following treatment with lovastatin and /or 5-azacytidine according to the manufacturer's protocol. Briefly, HeLa cell were treated with vehicle, $0.2 \mu \mathrm{M}$ of lovastatin, 5-azacytidine or lovastatin/5Azacytidine for 48 hrs. Genomic DNA was extracted using TriZol as per the manufacturer's instruction and the concentration measured by nanodrop software. The genomic DNA $(1 \mu \mathrm{g})$ was digested with a methyl sensitive, methyl dependent or methyl sensitive/dependent enzymes for $6 \mathrm{hrs}$. The digested DNA was them used as a template 
SBRY (BioRad, Hercules, CA) for qRT-PCR using the SREBF1 methyl primer. Methylation status was determined using the EpiTect methyl DNA methylation PCR data analysis tool (Qiagen, Frederick, MD).

\subsubsection{Statistics}

The experimental results were expressed as the mean \pm standard deviation of at least 3 independent experiments. Two-tail Student's t-test was performed with $\mathrm{p} \leq 0.05\left(^{*}\right)$ considered as statistically significant between the samples and their respective controls.

\subsection{RESULTS}

\subsubsection{Lovastatin up-regulates miR-33b expression and adversely impacts on c-Myc expression and function in medulloblastoma cells.}

We performed a small scale screening assay to identify FDA-approved compounds that reduce medulloblastoma cell viability and increase miR-33b expression using Daoy, a medulloblastoma cell line with intact $17 \mathrm{p} 11.2$ and with no gene amplification of $M Y C$ $(100,241)$. We singled out lovastatin (Fig. 17) as (i) its resulted in inhibition of Daoy cell proliferation by more than $40 \%$ and up-regulated miR-33b expression by 2.8 fold; (ii) its safety and efficacy has been tested for over thirty years; (iii) statins are reported to reduce cancer risk $(242,243)$; and (iv) statins or other cholesterol-lowering approaches up-regulate miR-33a, the homolog of miR-33b (244-247). MTT cell

proliferation/viability assay was performed after Daoy or D283 cells were treated with increasing concentration of Lovastatin. The decrease in cell proliferation was lovastatin dose-dependent in Daoy but did not affect the proliferation of D283 cells (Fig. 18). 


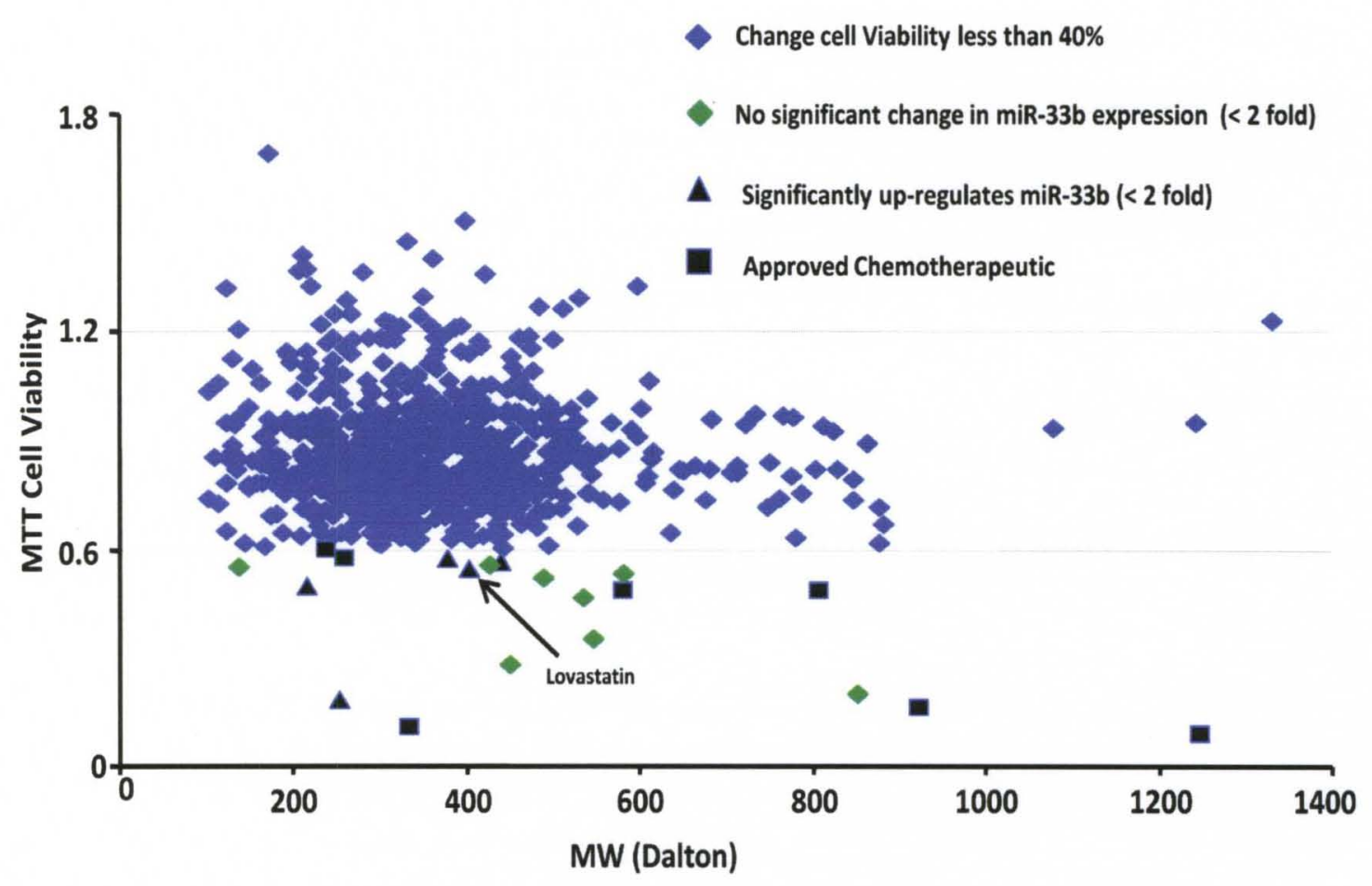

Fig. 17: Lovastatin most significantly resulted in reduced Daoy cell proliferation and up-regulation of miR-33. A small scale screening assay to identify FDAapproved compounds that reduce medulloblastoma Daoy cell proliferation and increase miR-33b expression. 


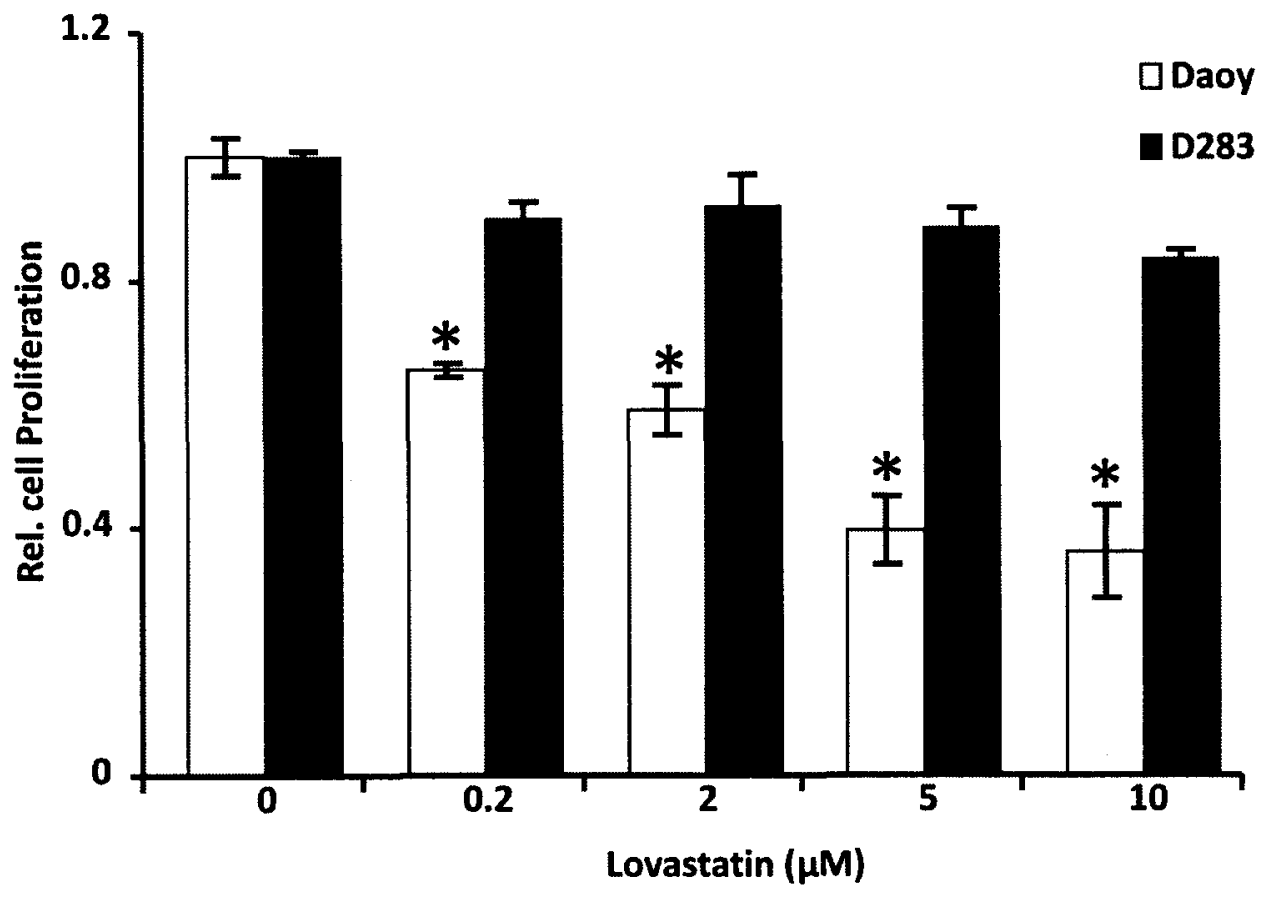

Fig. 18: Lovastatin dose-dependent decrease in Daoy, but not D283, cell proliferation. D283 and Daoy cell treated with lovastatin for 48 hrs followed by MTT cell proliferation/viability assay. 
A qRT-PCR assay showed that in Daoy cells, lovastatin induced miR-33b and SREBF1 expression in a dose-dependent manner, while MYC mRNA was down-regulated (Fig. 19A). Yet there was no significant change in $M Y C$ mRNA levels upon lovastatin treatment in D283 cells (Fig. 19C). We tested the promoter of the human SREBF1 miR$33 b$ gene and found that lovastatin increased the promoter activities (Fig. 20A). In addition, mevalonate inhibited the induction of miR-33b and $S R E B F 1$ and $M Y C$ mRNA down-regulation by lovastatin in Daoy cells (Fig. 20C), suggesting lovastatin activates miR-33b through the cholesterol biosynthetic pathway. In D283 cells, mevalonate did not affect the expression of $M Y C$ mRNA (Fig. 20B). Using other statins, we found that miR-33b expression was also increased but in a less significant manner compared to lovastatin (Fig. 21). 

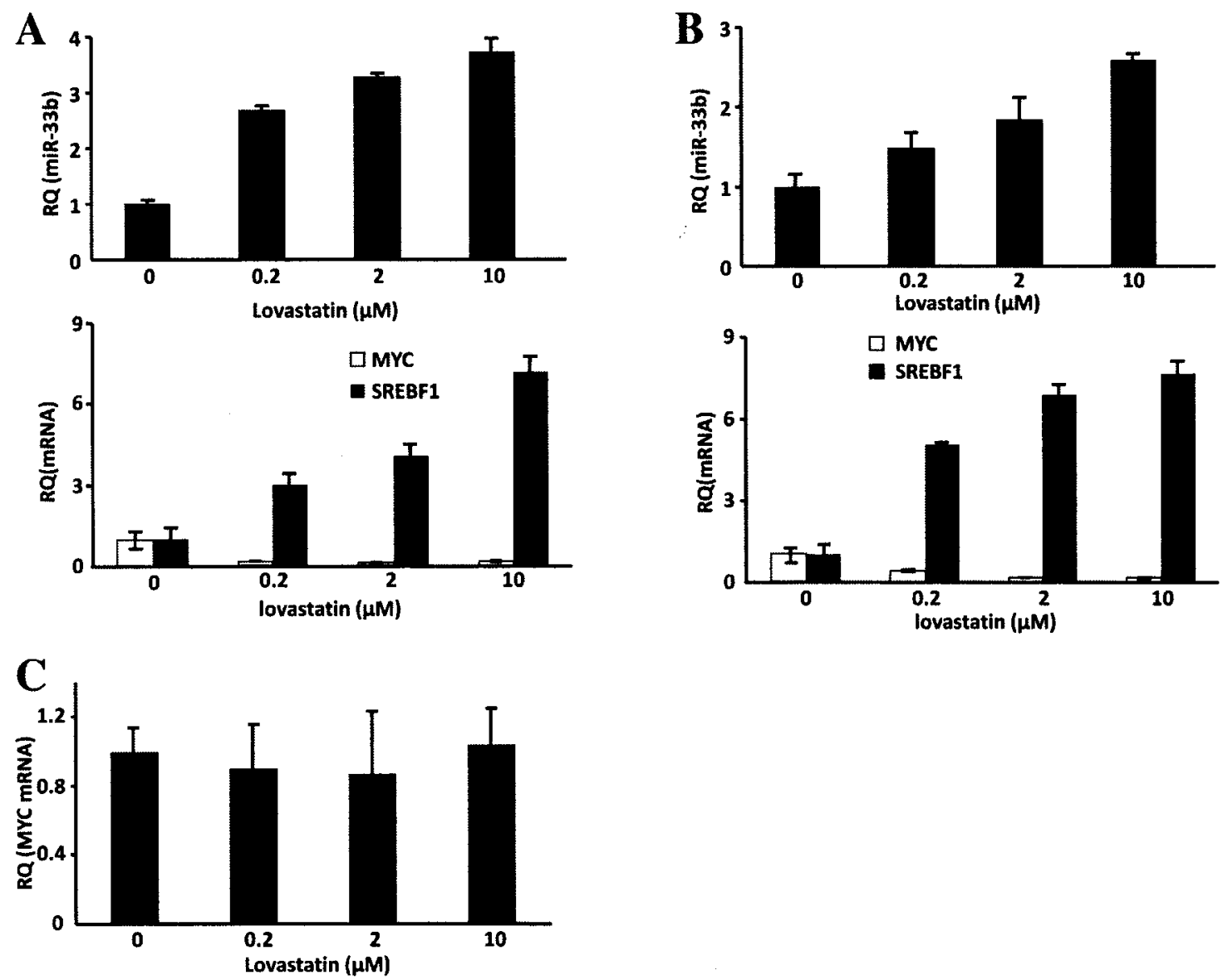

Fig.19: Lovastatin positively modulates miR-33b expression in miR-33b-positive medulloblastoma cells Daoy and UW228. (A) QRT-PCR to measure the levels of $m i R-33 b, S R E B F 1$ and MYC mRNA in Daoy cells treated with lovastatin for 48 hrs. (B) QRT-PCR to measure the levels of $m i R-33 b, S R E B F 1$ and $M Y C$ mRNA in UW228 cells treated with lovastatin for $48 \mathrm{hrs}$. (C) MYC mRNA expression in D283 cell treated with lovastatin was determined by TaqMan realtime PCR. 

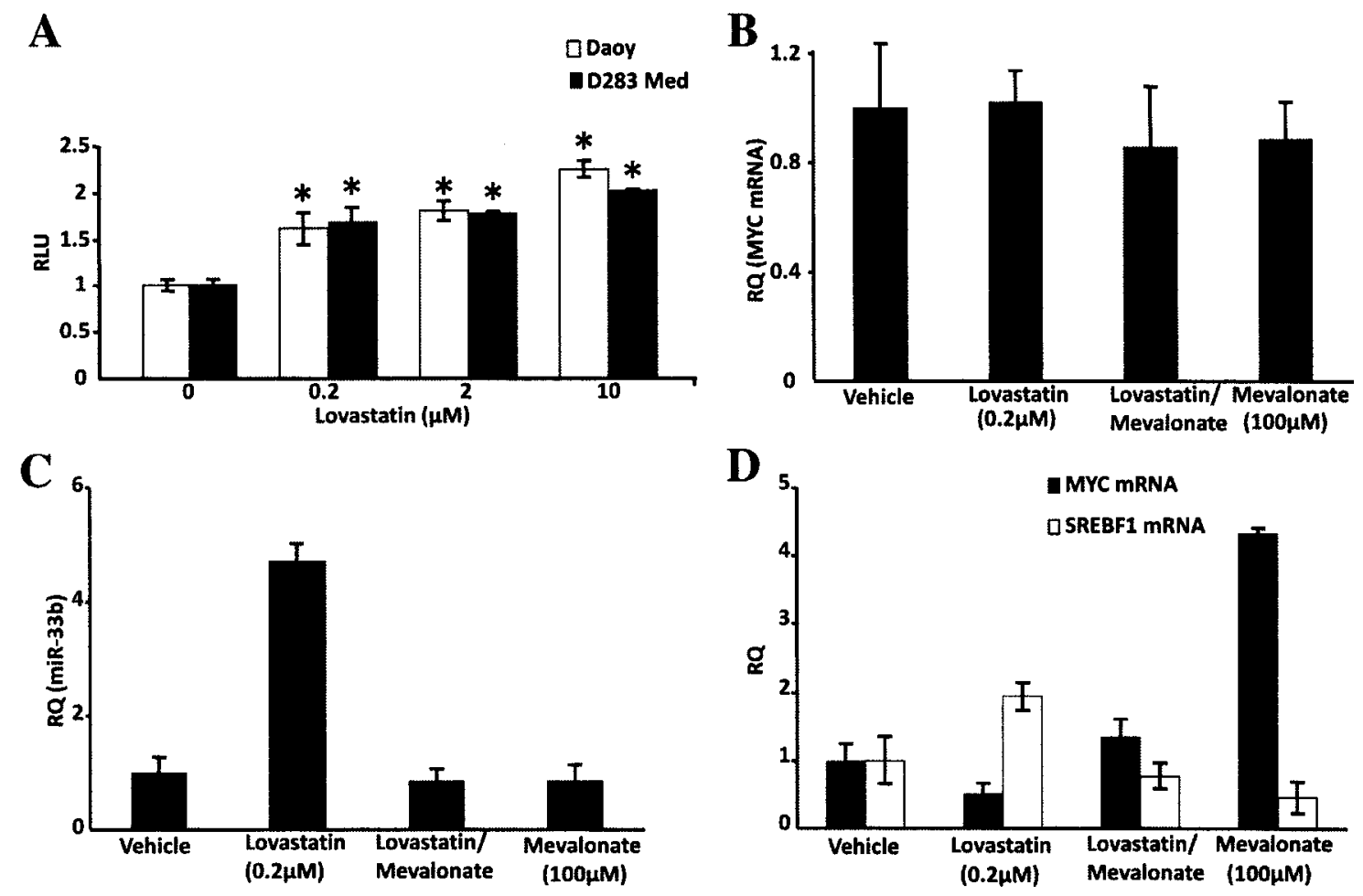

Fig. 20: Lovastatin increases miR-33b transcription through the cholesterol synthetic pathway. (A) Luciferase activity from an SREBF1promoter luciferase construct in Daoy and D283 cells treated in response to lovastatin. (B) MYC mRNA expression levels in D283 cells treated with lovastatin and/or mevalonate was determined by TaqMan qRT-PCR. (C \& D) TaqMan qRT-PCR was used to determine the expression levels of $m i R-33 b, S E R B F 1$ and $M Y C$ mRNA from Daoy cells treated with lovastatin and/or mevalonate. 


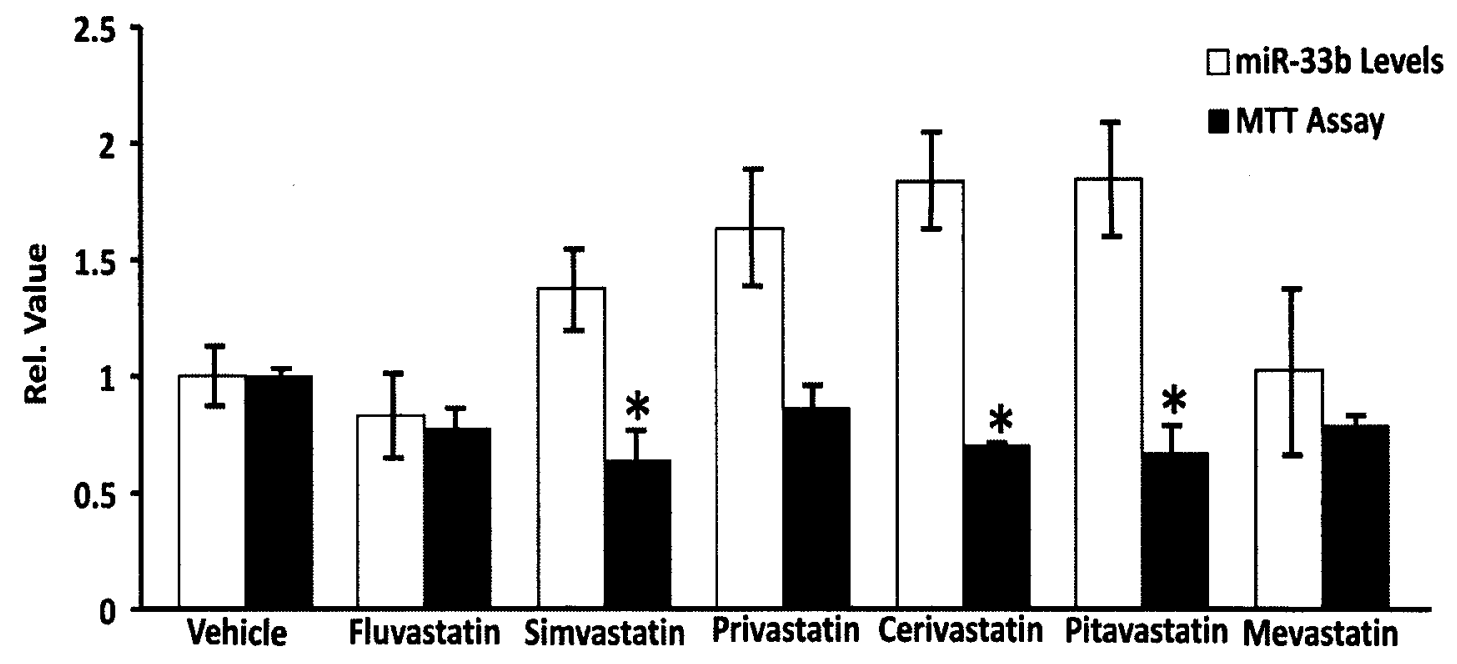

Fig. 21: Impact on cell proliferation and miR-33b expression by other statins in Daoy cells. Daoy cells were treated with $0.2 \mu \mathrm{M}$ of various statins, and cell proliferation and miR-33b levels were determined. miR-33b was up-regulated by Simvastatin, Pravastatin, Cerivastatin, and Pitavastatin. 
Correspondingly, lovastatin caused reduction in c-Myc, Cyclin E and ODC protein levels and up-regulation of Gadd45 $\alpha$ in Daoy (Fig. 22A) but not in D283 cells (Fig. 22B). We also treated UW228 (another cell line with an intact 17p11.2) with lovastatin and found there was a significant induction of miR-33b and SREBF1, and a decrease in MYC mRNA expression in UW228 cells (Fig. 19B). Equally, protein levels of c-Myc and its transcriptional targets Cyclin E and ODC are down-regulated in a dose-dependent manner by lovastatin in UW228 cells, while Gadd45a was up-regulated (Fig. 22A) This is in line with a previous report that Daoy and UW228 are more sensitive to lovastatin than D283 (248). Cell cycle analyses demonstrated that more Daoy but not D283 cells had delay G1 phase progression upon lovastatin treatment (Fig. 23). Lovastatin resulted in decreased levels of miR-9 (Fig. 24A) and a corresponding reduction in cell migration of Daoy cells (Fig. 24B) but did not affect miR-9 (Fig. 24A) levels or cell migration of D283 cells (Fig. 25B). 

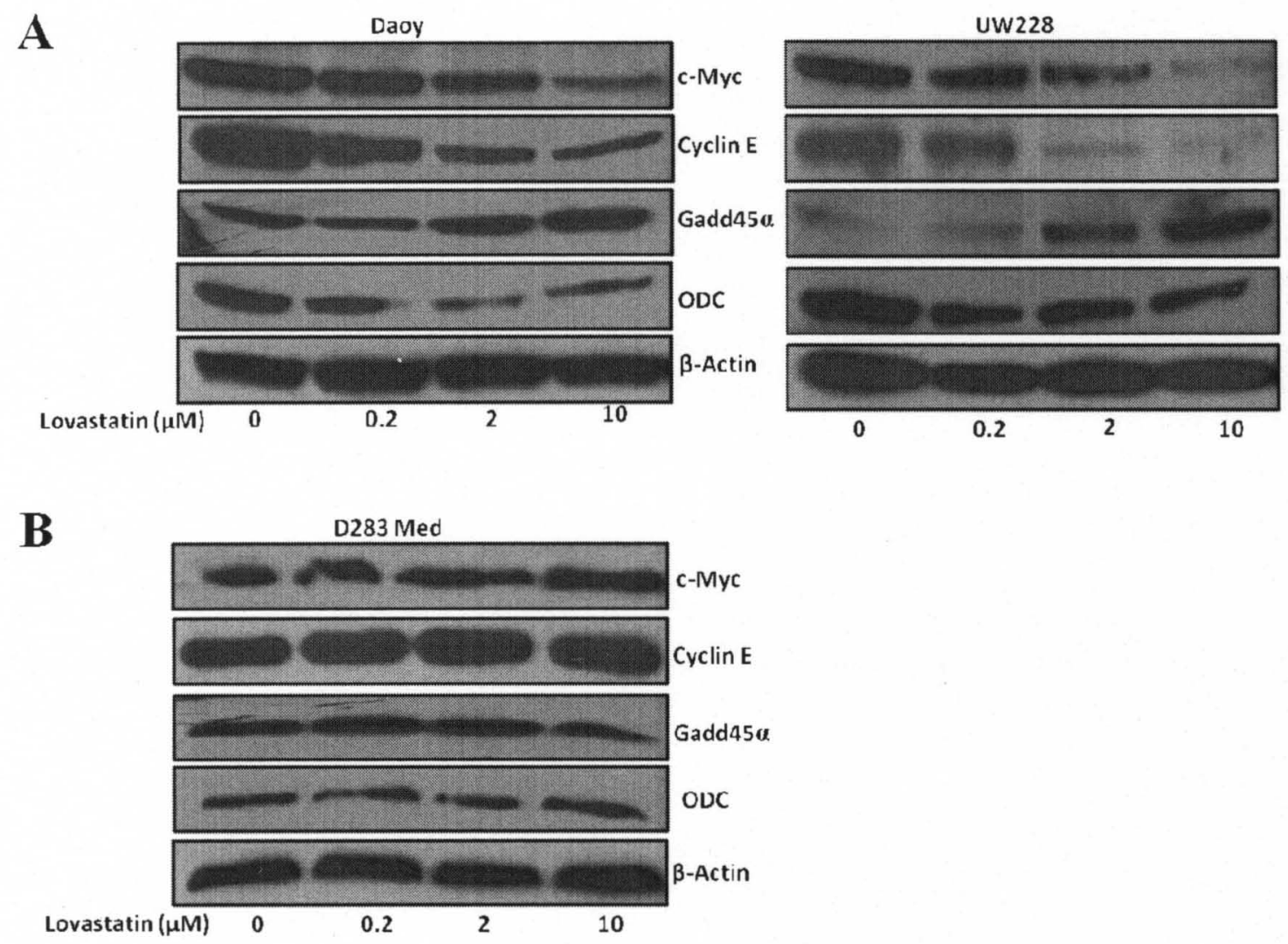

Fig. 22: Lovastatin down-regulates the protein expression of c-Myc and its transcriptional target in miR-33b-positive medulloblastoma Daoy and UW228 cells. (A) Western blot analysis for the expression of c-Myc, Cyclin E, ODC and Gadd45 $\alpha$ in response to lovastatin treatment of miR-33b-positive Daoy and UW228 cells. (B) Protein levels of c-Myc and its target Cyclin E, ODC and Gadd $45 \alpha$ determine by western blot in miR-33b-negative D283 cells upon lovastatin treatment. 

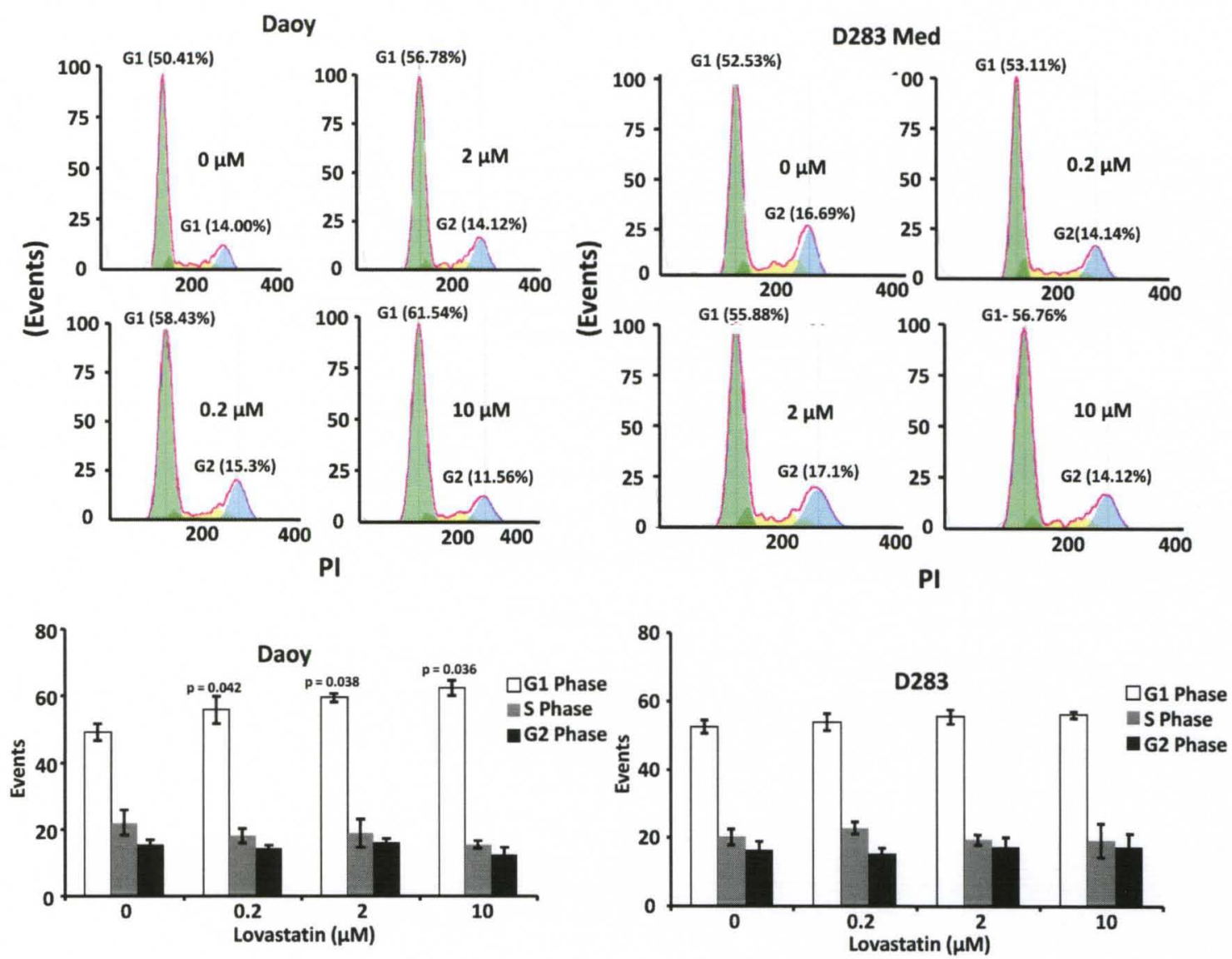

Fig. 23: Lovastatin increase G1 cell cycle arrest in Daoy, but not D283, cells. Cell cycle analyses of Daoy (left) and D283 (right) cells treated with lovastatin. Lovastatin induces significant G1 phase cell cycle arrest in Daoy cells but not D283 cells. On the top is a representative photo of a single run with the y-axis denoting events (the number of cells) and the $\mathrm{x}$-axis denoting the emitted fluorescence of the DNA dye (PI); the bar graph on at the bottom summarizes three independent runs. " $0-10 \mu \mathrm{M}$ " indicates the final concentration of lovastatin 

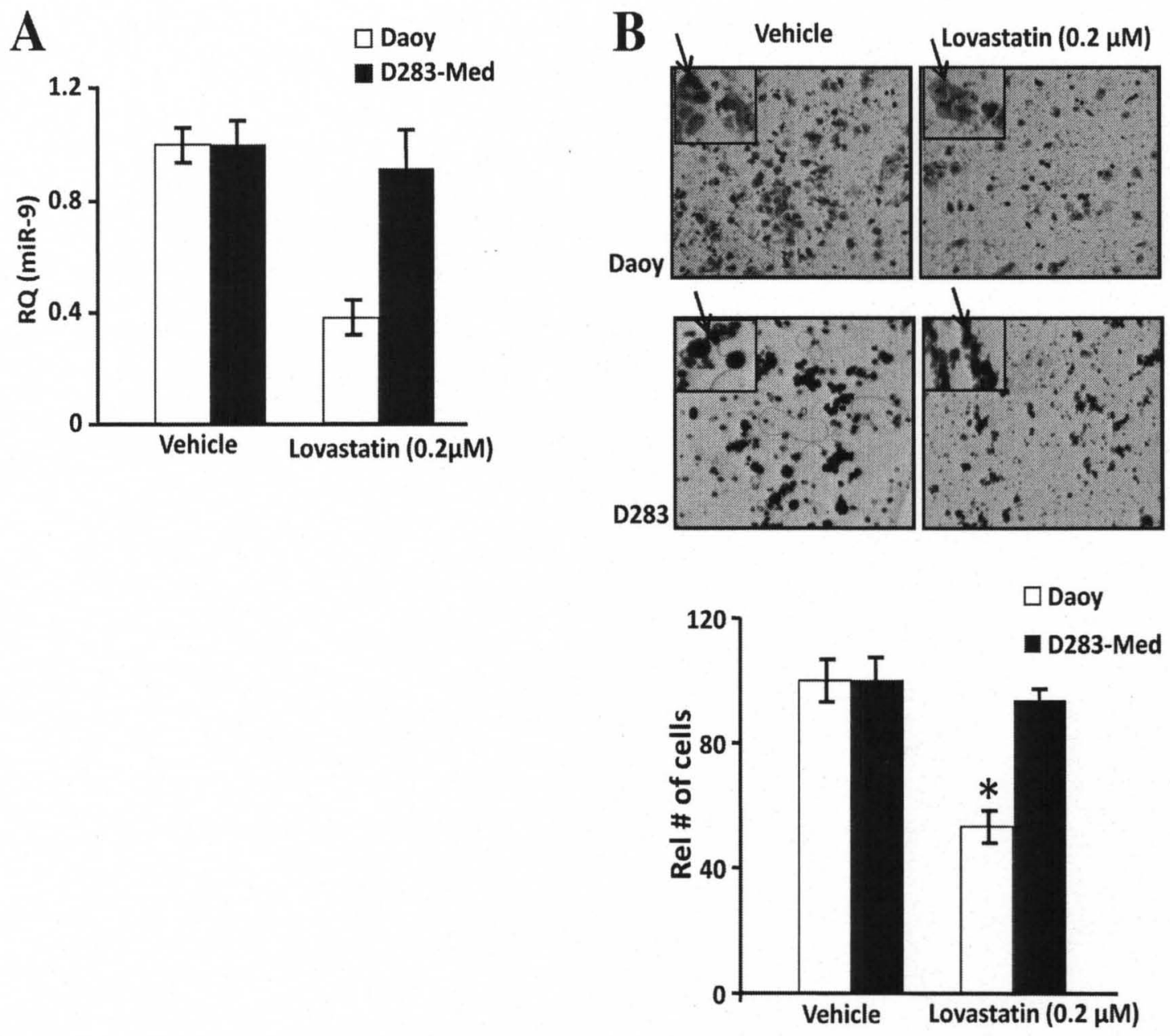

Fig. 24: Lovastatin decrease cell invasiveness of miR-33b-positive medulloblastoma

Daoy cells. (A) miR-9 expression levels, assayed by qRT-PCR, in Daoy and D283 cells treated with vehicle or lovastatin. Lovastatin results in downregulation of miR-9 in Daoy cells, but not D283. (B) Transwell cell migration assay of Daoy and D283 cells treated with vehicle or lovastatin. The upper panel is a representative photo of migrated cells; the bottom panel is the bar graph summarizing three independent experiments. 


\subsubsection{Lovastatin decrease tumor growth of miR-33b-postive medulloblastoma Daoy, but not $\mathrm{D283}$, cells in immunodeficient nu/nu mice}

We treated mice subcutaneously injected with Daoy cells to form xenografts and found either $1.0 \mathrm{mg} / \mathrm{kg}$ or $0.2 \mathrm{mg} / \mathrm{kg}$ of lovastatin treatment resulted in smaller tumor sizes (Fig. 25A) and lower c-Myc expression (Fig. 26D). We then tested whether lovastatin reduces the brain tumor burden in an orthotopic model, which requires testing compounds to penetrate the blood-brain barrier (BBB), a separation of circulating blood and cerebrospinal fluid in the central nervous system. Lovastatin is lipophilic and is able to penetrate the BBB, and this comes with some side effects such as sleep disturbances $(249,250)$. We inoculated Daoy or D283 cells into the brain of immunodeficient mice and treated them with $1.0 \mathrm{mg} / \mathrm{kg}$ of lovastatin. At the end of regimen (4 wks), all Daoybearing mice in the control group became moribund or paralyzed; yet mice in the lovastatin group appeared physically normal. We found that Daoy-xenografted mice treated with lovastatin had less tumor expansion in the ventricles; and strikingly, tumor invasion into the surrounding cerebellar tissues was completely blocked (Fig. 25B). In contrast, D283 xenograft neither invaded surrounding tissues nor responded to lovastatin treatment (Fig.25B). Lovastatin treatment led to miR-33b up-regulation (Fig. 26B) and lowered expression of miR-9 (Fig. 26A), c-Myc, and Cyclin E in tumors of Daoy (Fig. 26C) but not in that of D283 cells (Fig. 26A \& C). There was no statistically significant change in the percentage of PCNA-positive cells in control tumors versus treated Daoy tumors (Fig. 26C), suggesting cell cycle arrest rather than apoptosis is a likely cause for attenuated tumor growth in vivo. 
A

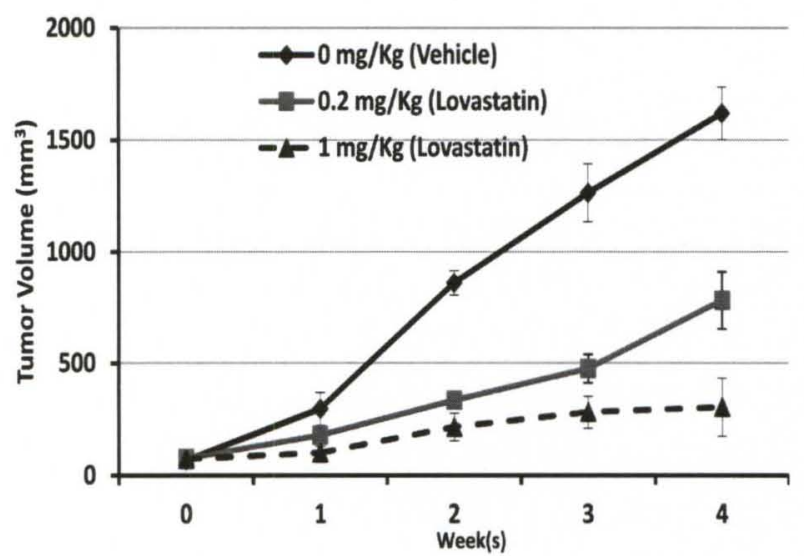

B
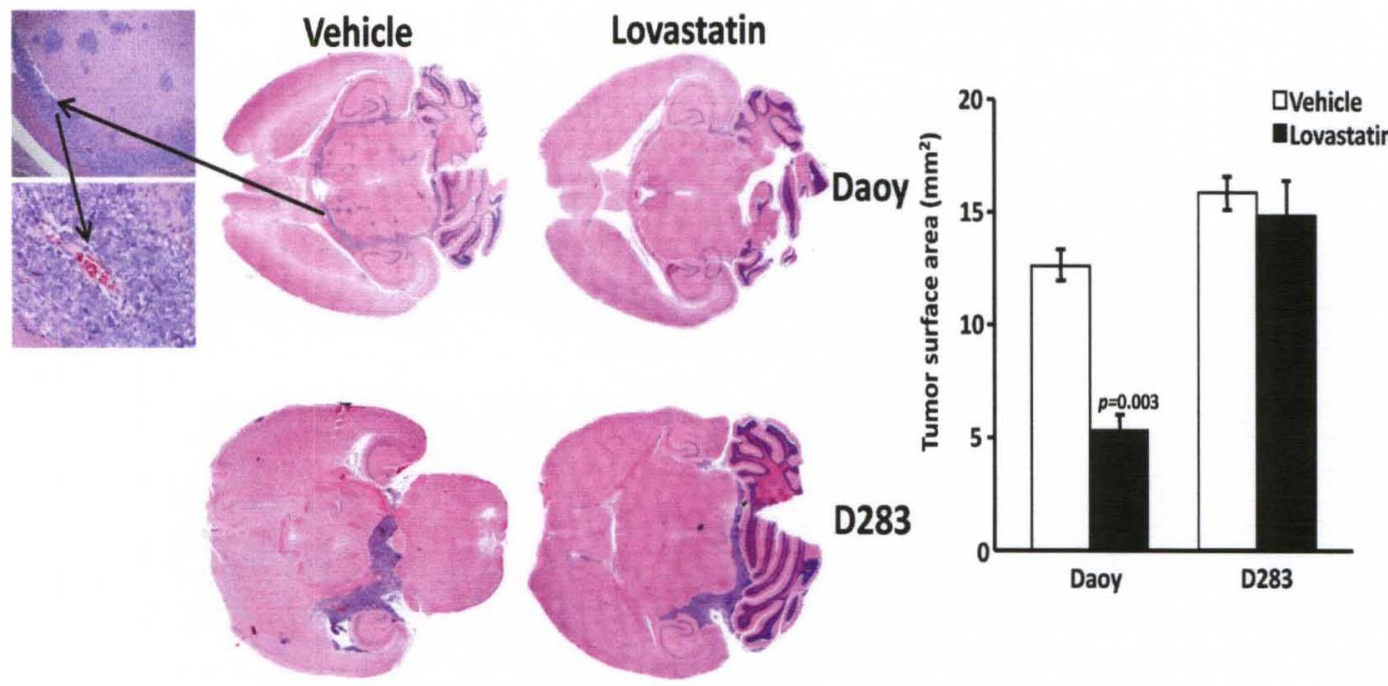

Fig. 25: Xenograft models of medulloblastoma with lovastatin treatment. (A)

Subcutaneous tumor growth rate of Daoy tumors in vehicle or lovastatin treated nu/nu mice. (B) A representative H\&E staining of the brain from mice, orthotopically injected with Daoy or D283 cells, treated with vehicle or lovastatin (left). Average surface area from tumors in vehicle or lovastatin treated group (Right) 

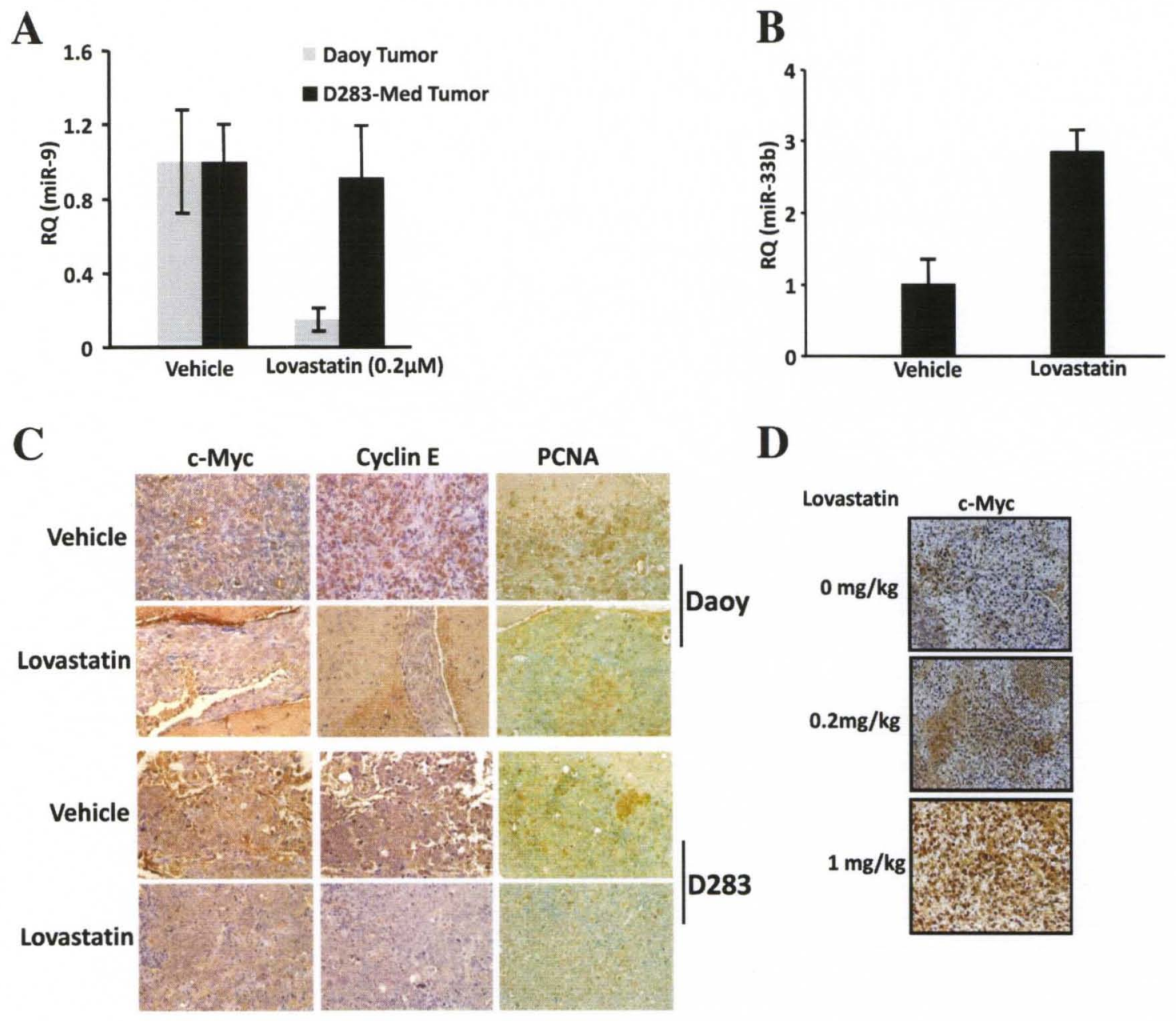

D

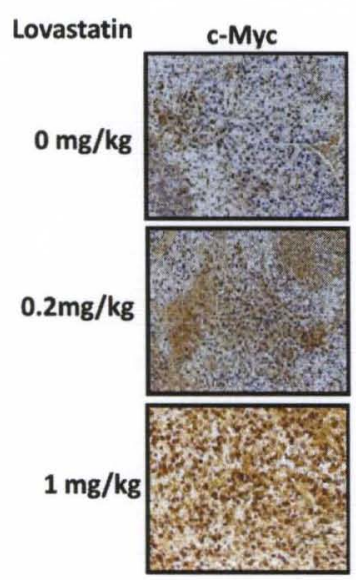

Fig. 26: Lovastatin treatment led to miR-33b up-regulation and lowered expression of miR-9, c-Myc, and Cyclin E in Daoy tumors. (A \& B) The expression of miR-9 from Daoy and D283 tumors from mice treated with vehicle or lovastatin (A) The levels of miR-33b from Daoy tumor from vehicle or lovastatin treated mice (B). (C) IHC analyses of orthotopic tumor of Daoy or D283 cells. (D) IHC analyses of c-Myc expression in subcutaneous Daoy tumor sections. 


\subsubsection{The methylation inhibitor 5-azacytidine sensitize HeLa cells to lovastatin- induced inhibition of cell growth}

There was no change in proliferation of HeLa cell treated with increasing concentration

of lovastatin $(0,0.2,2$, or $10 \mu \mathrm{M})$, except at the high concentration of $40 \mu \mathrm{M}$ (Fig. 27A), a concentration used to synchronize HeLa cells at G1 phase of the cell cycle (251). QRTPCR shows that in HeLa cells, lovastatin did not affect the levels of miR-33b and MYC mRNA (Fig. 27B \& C). Correspondingly, lovastatin did not affect the protein levels of cMyc and its target gene Cyclin E, ODC and Gadd45 (Fig. 27D). Cell cycle analysis revealed that at a concentration up to $10 \mu \mathrm{M}$ there was no change in cell cycle progression (Fig. 28B). Using FDA-approved drugs that were found to up-regulate miR33b in Daoy cells, qRT-PCR using RNA from HeLa cells treated with $0.2 \mu \mathrm{M}$ of these drugs did not significantly affect on miR-33b expression (Fig. 28A). 

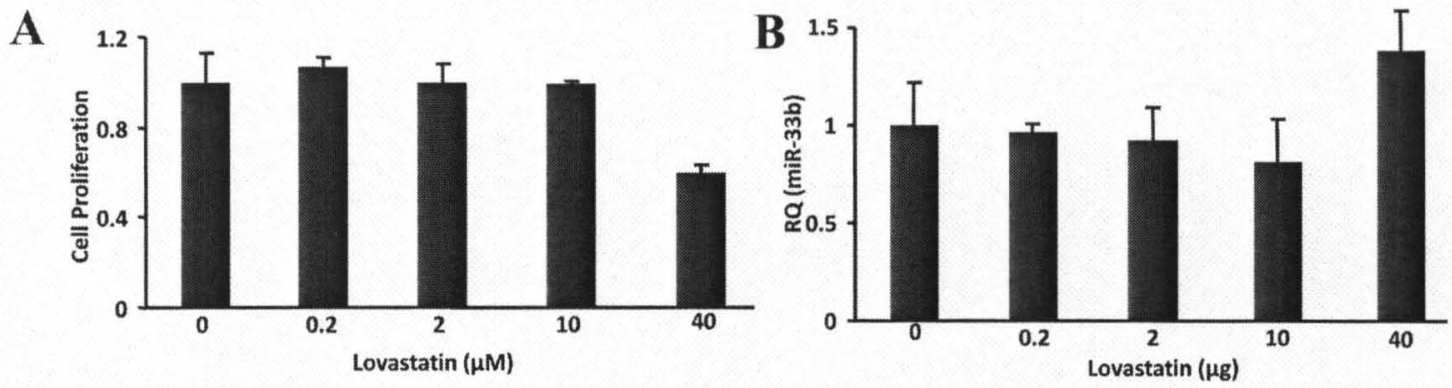

C

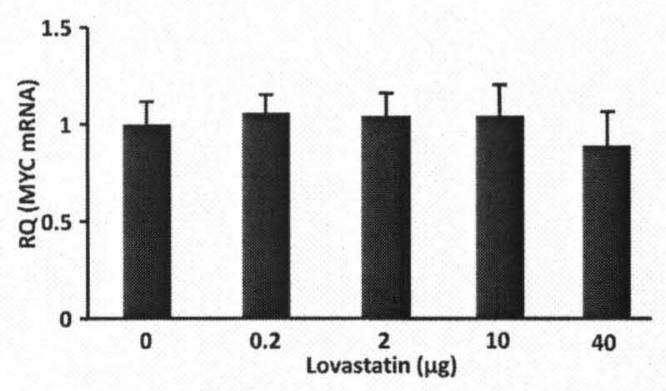

D

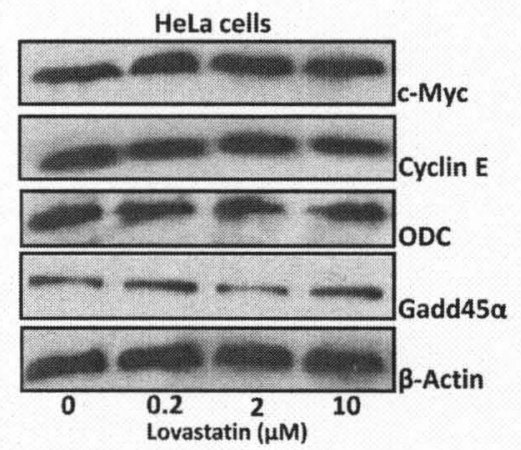

Fig. 27: HeLa cells show reduced sensitivity in response to lovastatin treatment. (A) MTT cell proliferation assay of HeLa cell in response to lovastatin treatment for 48 hrs. ' $0-10 \mu$ ' denotes low therapeutic concentration range; ' $40 \mu \mathrm{M}$ ' donates high concentration (B \& C) QRT-PCR assay shows the level of miR-33b (B) or MYC mRNA (C) in HeLa cells response to lovastatin. (D) Protein levels of cMyc and its transcriptional targets Cyclin E, ODC and Gadd45 $\alpha$ in HeLa cells upon lovastatin treatment for $48 \mathrm{hrs}$ determined by western blot. 


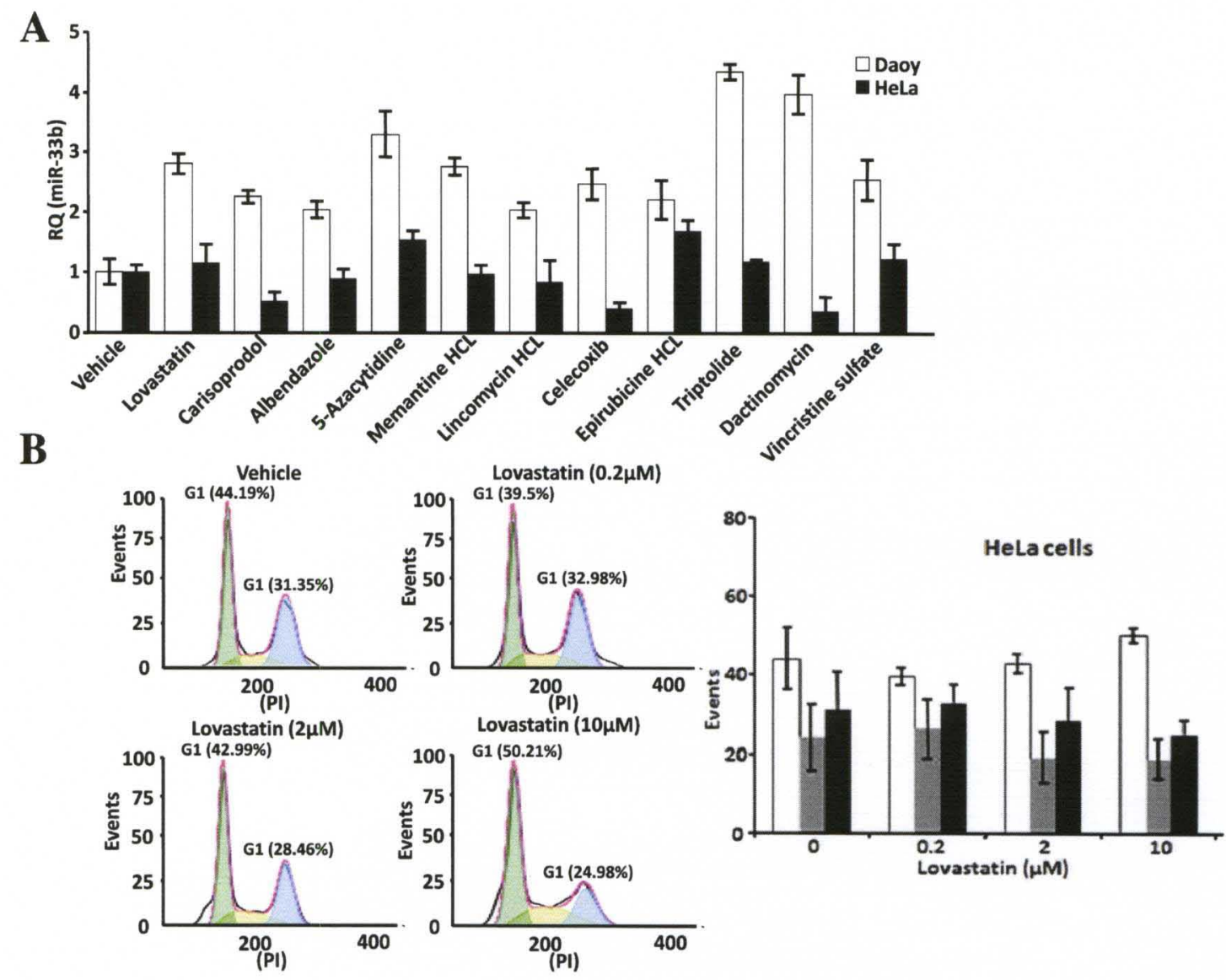

Fig. 28: Expression of miR-33b is lower in HeLa cells compared to Daoy cells in response to FDA approved drugs. (A) Differential expression of miR-33b in response to drugs that significantly decrease medulloblastoma Daoy cell proliferation. (B) Flow cytometry analysis for cell cycle progression of HeLa cell treated with lovastatin for $48 \mathrm{hrs}$. On the left is a representative photo of a single run with the $y$-axis denoting events (the number of cells) and the $\mathrm{x}$-axis denoting the emitted fluorescence of the DNA dye (PI); the bar graph on the right summarizes three independent runs. "0-10 $\mu \mathrm{M}$ " indicates the final concentration of lovastatin. 
HeLa cell proliferation was significantly reduced in response to valproic/lovastatin acid or 5-azacytidine/lovastatin treatments compared to lovastatin, valproic acid, 5-azacytidine or vehicle treatment alone (Fig 29A). The reduced proliferation corresponded with upregulation of miR-33b expression and decreased MYC mRNA (Fig 29B \& C). Furthermore, c-Myc, Cyclin E and ODC protein expression levels were down-regulated, and Gadd45 $\alpha$ expression was up-regulated in HeLa cells treated with 5azacytidine/lovastatin (Fig. 29D). Cell cycle analysis show that 5-azacytidine/lovastatin increased G1 cell cycle arrest (Fig. 30B). A methylation assay demonstrates that 5azacytidine decrease the methylation status of the $S R E B F 1-m i R-33 b$ promoter to levels comparable to lovastatin sensitive Daoy cells (Fig. 30A). These results suggest that methylation on the $S R E B F 1-m i R-33 b$ promoter might contribute to reduced lovastatin sensitivity in HeLa cells. 

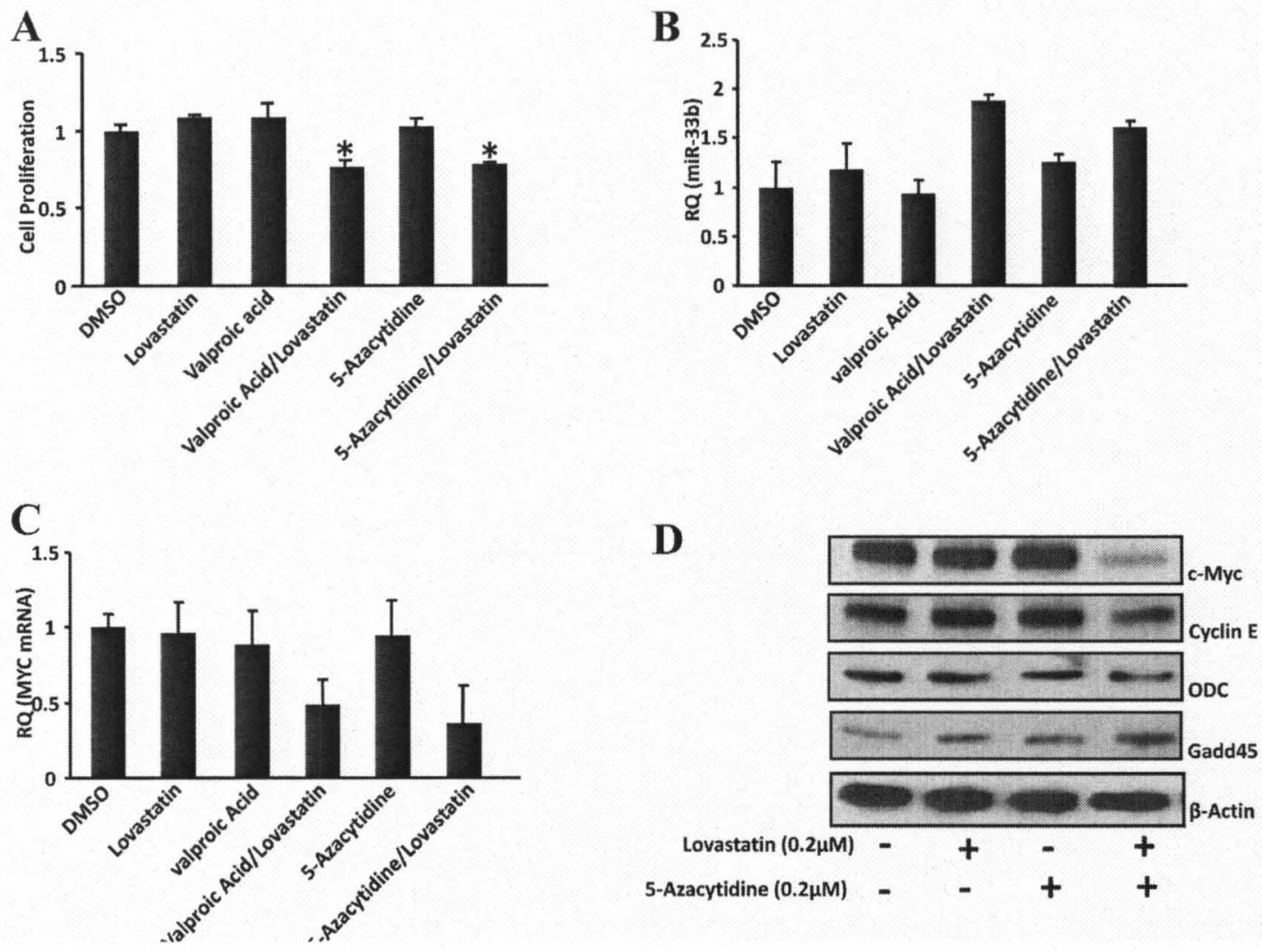

Fig. 29: 5-Azacytidine sensitizes HeLa cells to lovastatin-induced inhibition of cell proliferation. (A) Cell proliferation of HeLa cells was determined by MTT cell proliferation assay following treatment with vehicle, lovastatin and/or 5azacytidine for $48 \mathrm{hrs}$. (B \& C) HeLa cells expression of miR-33b (B) and MYC mRNA (C) assayed by qRT-PCR upon vehicle, lovastatin and/or 5-azacytidine treatment. (D) Western blot analysis for expression of c-Myc, Cyclin E, ODC and Gadd $45 \alpha$ in HeLa cell treated with lovastatin and/or 5-azacytidine for 48 hrs. 


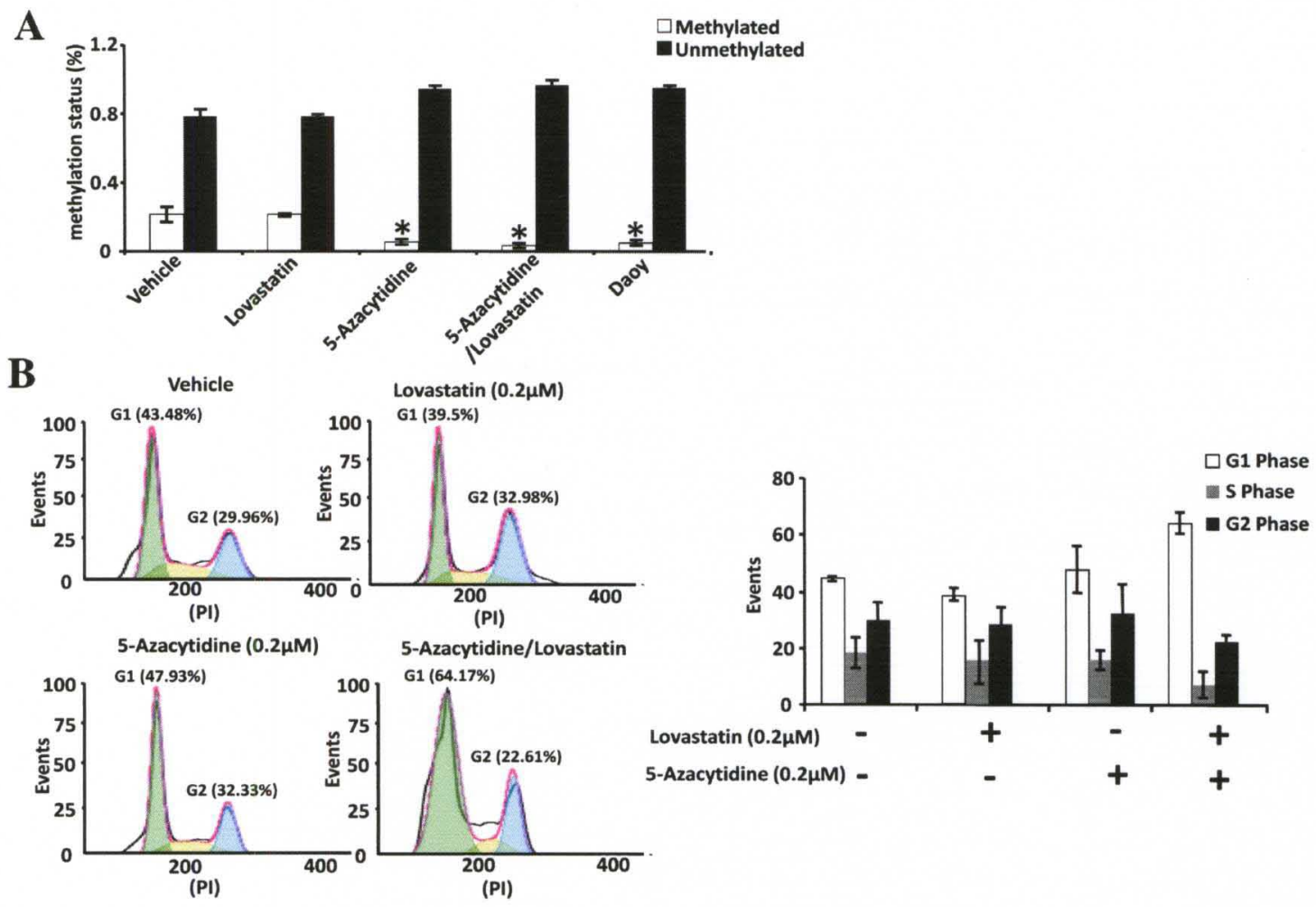

Fig. 30: 5-Azacytidine treatment decreases the methylation level of the SREBF1miR-33b gene enable lovastatin-mediated G1 cell cycle arrest of HeLa cells. (A) Methylation status was determined by SYBR green PCR using SREBF1 methyl specific primer following enzymatic digestion of HeLa cell genomic DNA extracted from cells treated with vehicle, lovastatin and/or 5-azacytidine (B) Flow cytometry analysis for cell cycle progression of HeLa cell treated with vehicle, lovastatin and/or 5-azacytidine. On the left is a representative photo of a single run with the $y$-axis denoting events (the number of cells) and the $x$-axis denoting the emitted fluorescence of the DNA dye (PI); the bar graph on the right summarizes three independent runs. "0-10 $\mu \mathrm{M}$ " indicates the final concentration of lovastatin. 


\subsection{4. miR-33b down-regulates endogenous c-Myc expression and adversely affect its}

\section{function in HeLa cells}

Next, we determined the expression of c-Myc and its transactivational targets in HeLa

cells following miR-33b over-expression. We found down-regulation of c-Myc, Cyclin E, ODC (Fig. 31A), and miR-9 (Fig. 33A), a miRNA regulated by c-Myc (252) and upregulation of Gadd45 $\alpha$ (Fig. 31A) by miR-33b but not by miR-33bM in HeLa cells, similar to that in $293 \mathrm{~T}$ cells. Additionally, $M Y C \mathrm{mRNA}$ levels were reduced in response to miR-33b over-expression (Fig. 31B). 
A
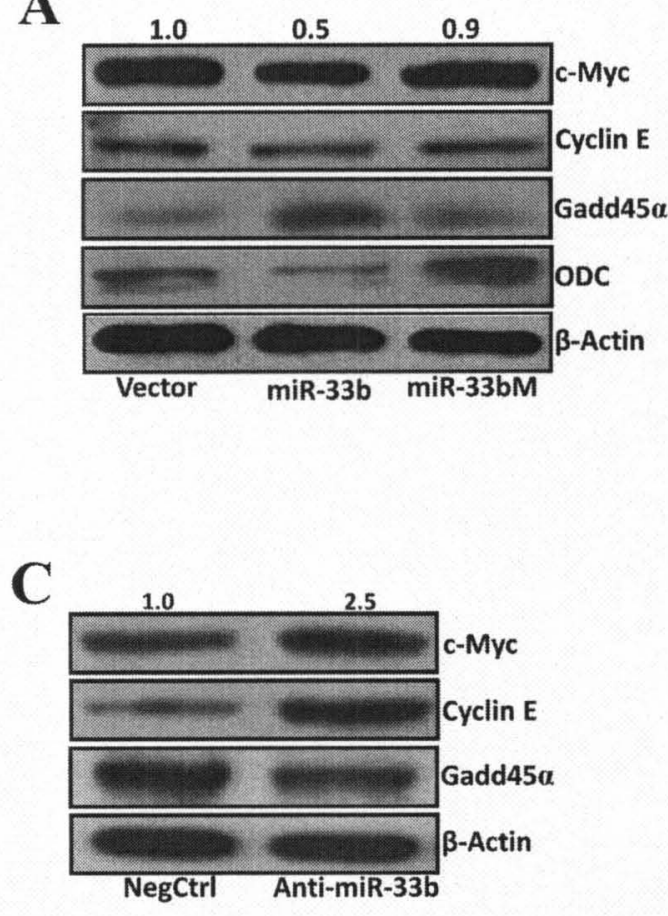

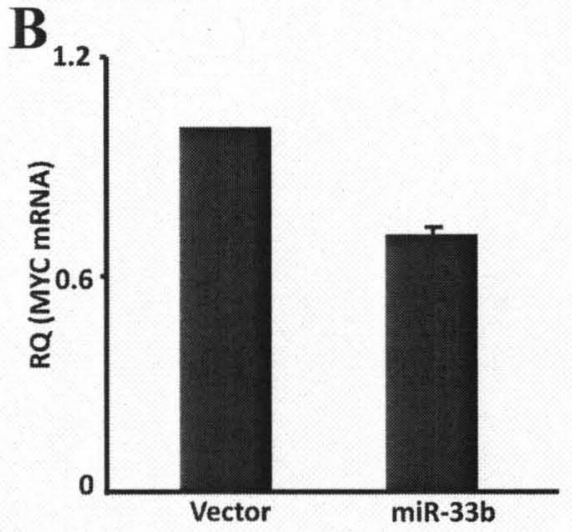

Vector miR-33b 
As cyclin E expression is required for G1-S phase transition during cell cycle, we performed cell cycle analyses using flow cytometry. As shown in Fig. 32A, miR-33b, but not miR-33bM or the control resulted in more cells arrested at the G1 phase. Moreover, as c-Myc is reported to promote cell proliferation, and cell migration (100, 211-213, 215), we performed MTT, and Transwell assays to determine whether these cMyc functions in HeLa cells were compromised by miR-33b. As expected, cells with miR-33b over-expression have a significant reduction in cell proliferation (Fig. 32B), and cell migration (Fig. 33B), indicating miR-33b down-regulate endogenous c-Myc expression and adversely affect c-Myc function in HeLa cells. We also inhibited miR$33 \mathrm{~b}$ expression using modified antisense oligonucleotides against miR-33b (Anti-miR33b) and found that the expression of c-Myc was up-regulated, so did cyclin E (Fig. 31C), amnd miR-9 (Fig. 33A) whereas Gadd45 $\alpha$ was down-regulated (Fig. 31C). Cell migration was increased when miR-33b was inhibited (Fig. 33B). We next determine whether exogenous expressed c-Myc could rescue apoptosis induced by miR-33b. We co-transfected cells with a c-Myc-expressing construct with a WT or a mutant 3'UTR and miR-33b. Flow cytometry results show that exogenous c-Myc, with the mutant UTR (which is not targeted by miR-33b) but not that with WT, is able to rescue apoptosis caused by miR-33b over-expression (Fig. 34). These results demonstrate that miR-33b overexpression negatively affects HeLa cells c-Myc-mediated functions. 

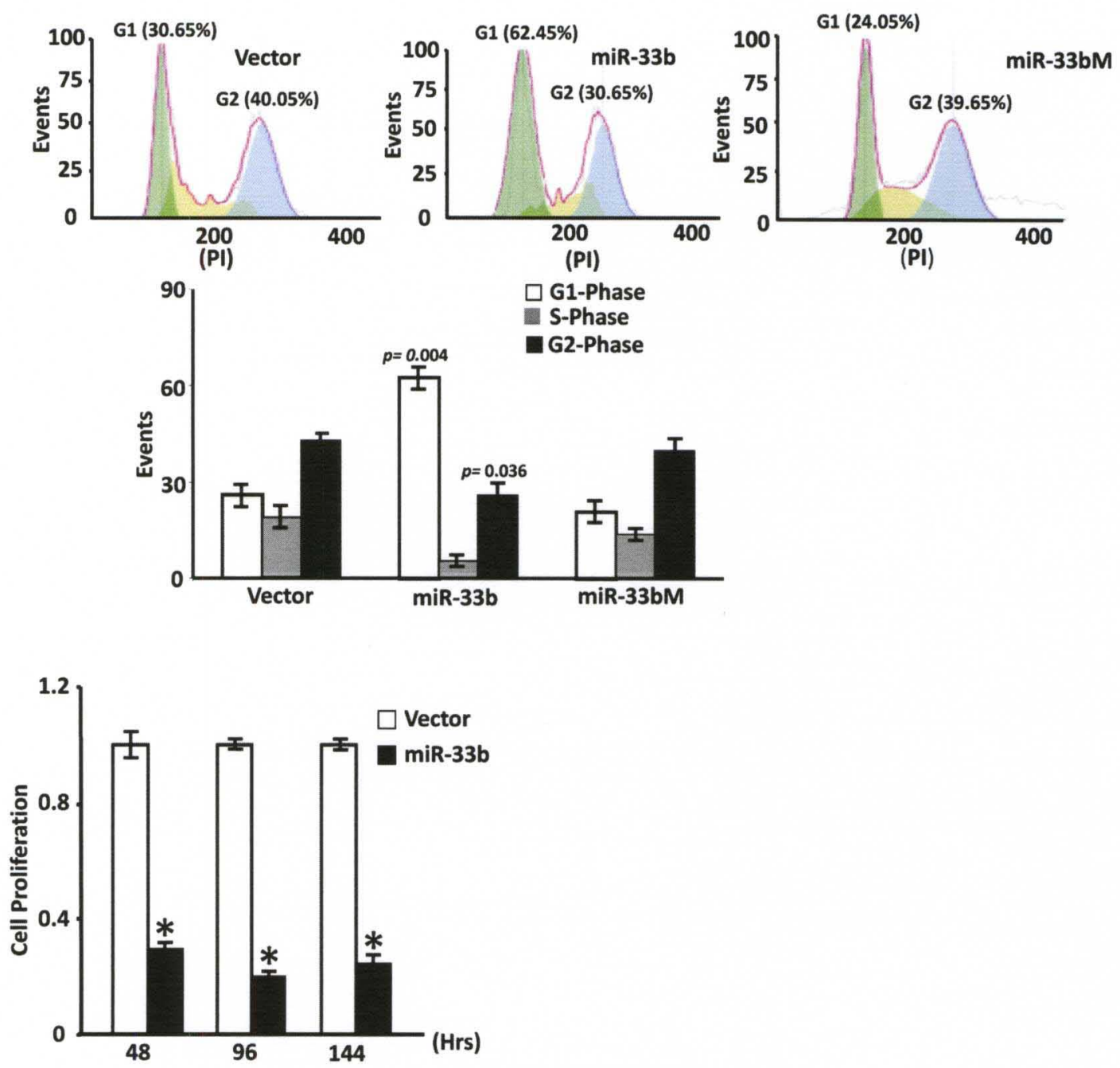

Fig. 32: Over-expression of miR-33b down-regulated c-Myc-mediated functions in

HeLa cells. (A) Flow cytometry of HeLa cells transfected with a parental vector, miR-33b or miR-33b mutant to determine cell cycle progression. On top is a representative photo of a single run with the y-axis denoting events (the number of cells) and the $\mathrm{x}$-axis denoting the emitted fluorescence of the DNA dye (PI); the bar graph at the bottom summarizes three independent runs. (B) HeLa cell proliferation following HeLa cell transfection with the parental vector or miR-33b for 48, 96 and 144 hrs. 

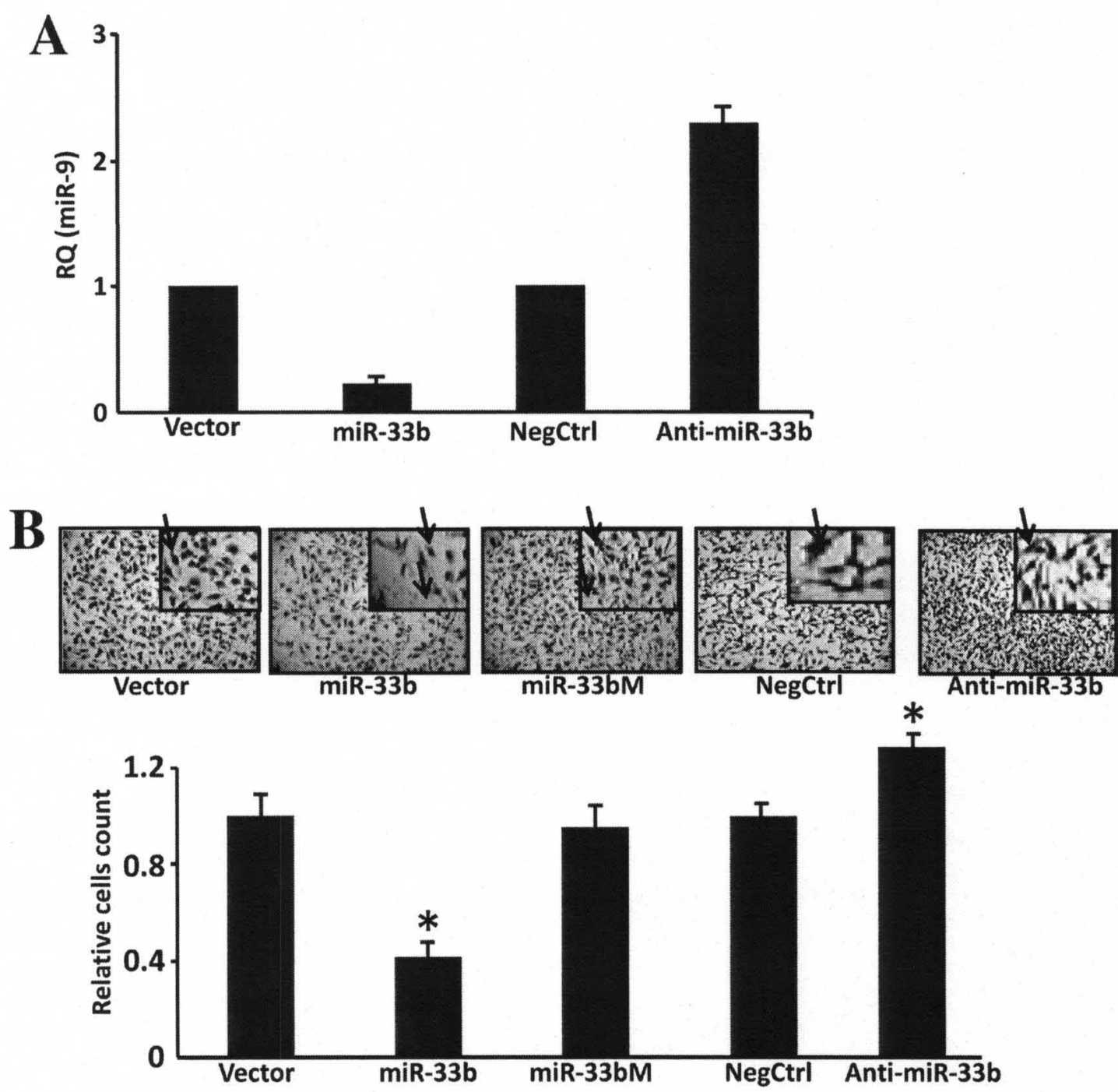

Fig. 33: Modulation of miR-33b inversely correlates with HeLa cells invasiveness.

(A) Expression level of miR-9 in HeLa cells determined by TaqMan qRT-PCR. (B)

Transwell cell migration assay of HeLa show that miR-33b expression inversely correlates cell migration. 


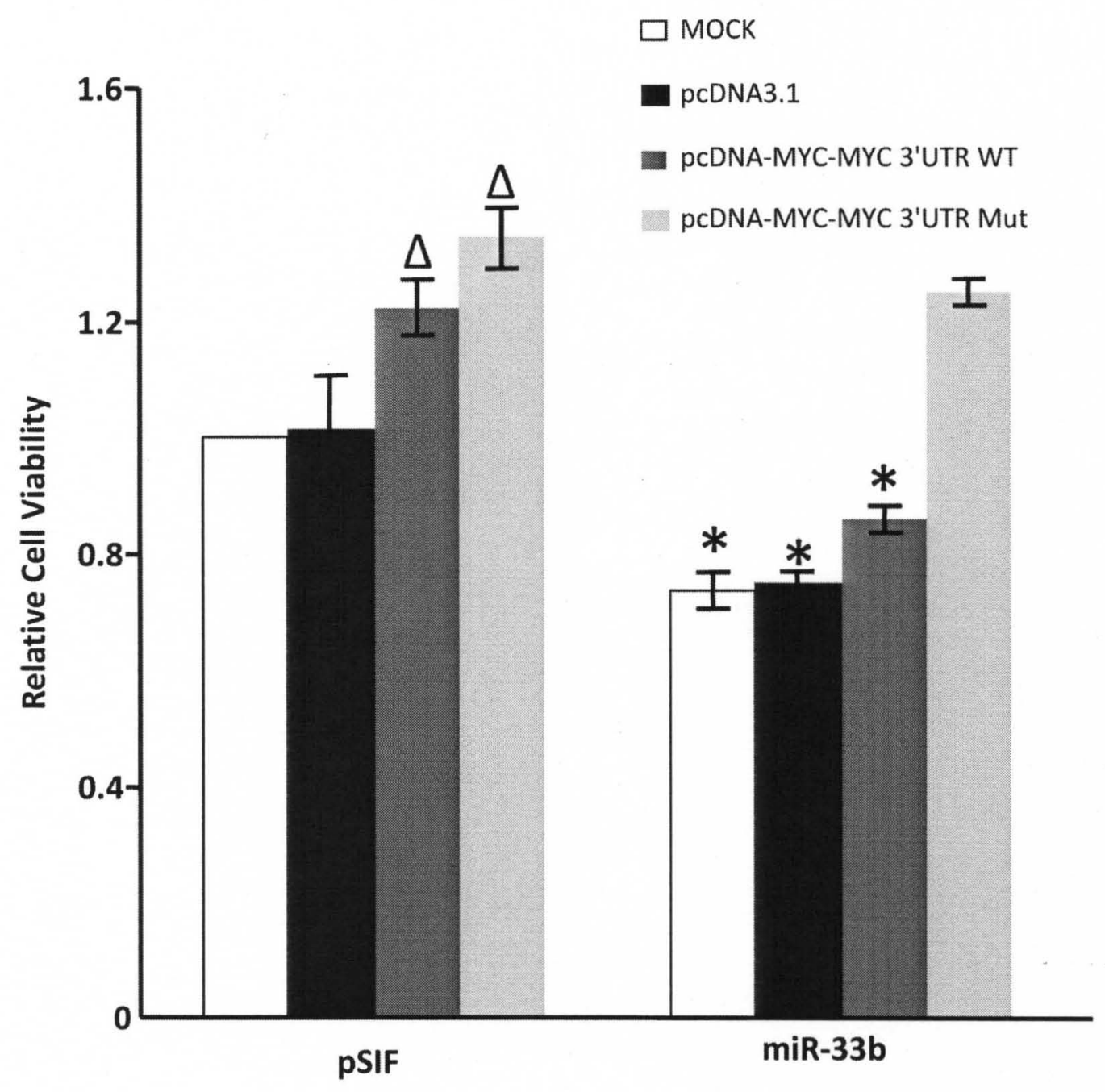

Fig. 34: miR-33b-induced apoptosis is rescued by exogenous c-Myc expression ( $\Delta$ indicates significance of $<0.05$ between pSIF transfected group: * represent significance of $<0.05$ between $\mathrm{pSIF}$ and miR-33b transfected groups). HeLa cells were co-transfected with MYC 3'UTR WT or mutant constructs and pSIF or miR-33b and 48 hrs later, flow cytometry was performed to determine cellular apoptosis. 


\subsection{DISCUSSION}

Lovastatin, a 3-hydroxy-3-methylglutaryl coenzyme A (HMG-CoA) reductase inhibitor, blocks the mevalonate pathway, decreasing cholesterol biosynthesis as well as the production of non-steroidal mevalonate derivatives (253). Lovastatin has profound cellular effects, including inhibition of proliferation and induction of apoptosis of cancer cells $(254,255)$, including medulloblastoma cells $(256)$, and has been used as a potential anticancer drug in clinical studies $(257,258)$. In this study, we show that lovastatin can be used against tumors through modulation of an miRNA targeting c-Myc. As described earlier, medulloblastoma show over-expression of c-Myc in $64 \%$ of cases but only 10$15 \%$ show MYC genomic amplification. The second major characteristic is that a subset $(\sim 17 \%)$ has a major breakpoint on chromosome $17 \mathrm{p} 11.2$, which results in chromosomal deletion at the breakpoint (259). One of the genes found in this deleted region is SREBF1 miR-33b, which is important in lipid metabolism and harbors miR-33b on intron 17. Interestingly, medulloblastoma cells that have this chromosome breakpoint show lower sensitivity to lovastatin treatment (256). We show that lovastatin treatment of medulloblastoma cell line Daoy results in a significant reduction in cell proliferation, an effect not observed in D283 cells. Lovastatin-induced inhibition of Daoy cell proliferation correlated with an increase in miR-33b expression as well as a downregulation of c-Myc expression. Treatment of mice with lovastatin resulted in a significant reduction of Daoy tumor growth. Concordantly, the tumors expressed lower levels of miR-9, c-Myc and Cyclin E. This, to our knowledge, is the first evidence of pharmaceutical modulation of c-Myc via a miRNA, and provides a new approach to cancer therapy by targeting miRNA with repurposed drugs. 
Sensitivity to lovastatin is cell-type specific (260). In the case of HeLa cells, lovastatin treatment has no effect on miR-33b expression. We hypothesize that epigenetic silencing of the $S R E B F 1$ gene promoter is responsible for the reduced lovastatin sensitivity. In mammals, the best understood epigenetic modification of DNA is post-replicative methylation of the $\mathrm{C} 5$ position of cytosine residues in $\mathrm{CpG}$ dinucleotides. We did find the SREBF1 promoter was significantly methylated in HeLa cells, but not in Daoy cells. 5-Azacytine is an FDA-approved antitumor drug that acts as a methyltransferase inhibitor, that prevents epigenetic gene silencing $(261,262)$. When HeLa cells were treated with 5-Azacytidine along with lovastatin, miR-333b was up-regulated and MYC was down-regulated. This was accompanied with decreased methylation of the SREBF1 promoter, as well as suppression of c-Myc-mediated cell proliferation, cell migration and cell cycle progression.

In summary, we have documented here that lovastatin represses the oncogenic activities of $c-M y c$ through activation of miR-33b transcription. Furthermore, lovastatin sensitivity may be augmented in cells that are resistant with the methylase inhibitor 5-Azacytidine. 


\section{CHAPTER 4}

\section{CONCLUDING REMARKS}

Tumor development is hallmarked by a variety of genetic and epigenetic alterations in protein-coding genes and small non-coding RNA genes. $M Y C$ is a major oncogene encoding the transcription factor c-Myc. miRNAs are a diverse family of small RNAs that regulate the stability and translational efficiency of partially complementary target mRNAs $(263,264)$. By regulating specific oncogenes or tumor-suppressor molecules, these small RNAs may have profound effects in tumor development (265). A few target genes have been validated for some miRNAs, and each individual miRNA can target multiple genes and participate in diverse physiological or pathological functions. In this study we aimed at elucidating miRNAs that target MYC. Using an array of molecular techniques including reporter assays, western blot analysis, cell cycle analysis, cell migration and cell proliferation assays, we showed that $M Y C$ is a miR- $33 \mathrm{~b}$ target gene.

Medulloblastoma is characterized by an over-expression of c-Myc. While $64 \%$ of medulloblastomas have over-expression of c-Myc, yet only 5-15\% of medulloblastomas show MYC gene amplification $(96,266)$. Myc over-expression is a biomarker associated with poor prognosis of medulloblastoma, along with chromosome $17 \mathrm{p}$ breakpoint abnormalities. Interestingly, Myc over-expression without genomic amplification is mostly observed in the more aggressive and metastatic anaplastic subtype of medulloblastoma $(96,267-269)$. The human $m i R-33 b$ gene is located in intron 17 of the 
SREBF1-miR-33b gene and this genomic locus, $17 \mathrm{p} 11.2$, is frequently lost in medulloblastoma $(44,228,270)$. Breakpoints on chromosome $17 \mathrm{p}$, similar to that in Smith-Magenis syndrome, present in medulloblastoma either leads to complete deletion of this chromosomal regions or it is translocated to chromosome $17 \mathrm{q}$ thereby forming an isochromosome $\mathrm{i} 17 \mathrm{q}$ (227). We have determined that miR-33b targets MYC and adversely affects its biological functions in medulloblastoma. Therefore the loss of miR$33 \mathrm{~b}$ expression could in part explain the over-expression of c-Myc in a subset of medulloblastoma. Various functional assays were used to show that re-introduction of miR-33b in anaplastic medulloblastoma D283 cells negatively affects medulloblastoma tumorigenic proporties by reducing cell proliferation, cell migration, and cell cycle progression. Using neurosphere assays, immucytochemistry and qRT-PCR, we show that miR-33b re-introduction results in reduction of neurospheres formed by medulloblastoma D283 cells and down-regulation of stem cell markers such as CD133, SOX2, Musashi and MEKL.

We decided to use drug repurposing to elevate miR-33b and inhibit c-Myc. We performed a small scale screening of FDA-approved drugs and found that lovastatin inhibits cell growth and increases miR-33b levels in Daoy cells. Lovastatin downregulated c-Myc expression and led to G1 arrest in the miR-33b-positive Daoy cells, but not in the miR-33b-null D283 cells. The change in miR-33b expression upon lovastatin treatment reciprocally corresponded with c-Myc expression. These results suggest that lovastatin acts in part as an activator of miR-33b expression, which, in turn, decreases cellular proliferation through suppression of the $M Y C$ gene. We then tested whether 
lovastatin reduces the brain tumor burden in an orthotopic model, which requires the testing compounds to penetrate the blood-brain barrier (BBB), a separation of circulating blood and cerebrospinal fluid in the central nervous system. Lovastatin is lipophilic and is able to penetrate the BBB $(250,271)$. We found that lovastatin slows the growth of tumors inoculated with Daoy, but not that with D283 (a miR-33b-negative cell line). This suggests a therapeutic option against medullablastoma with lovastatin using miR$33 \mathrm{~b}$ as a biomarker.

However, not all cell lines are responsive to lovastatin, one of which is HeLa. This could be due to hypermethylation of the promoter to $S R E B F 1$, the host gene for miR-33b (272). We did find that this promoter is methylated at a higher level in HeLa compared with Daoy cells. When treated with a methylase inhibitor, 5-azacytidine, HeLa cells became sensitive to lovastatin, along with reduced methylation of the promoter. In addition, increased sensitivity to lovastatin correlated with up-regulation of miR-33b and down-regulation of c-Myc expression in HeLa cells treated with 5-azacytindine. These results suggest that DNA hypermethylation of the SREBF1 promoter is the underline mechanism of lovastatin insensitivity in HeLa cells.

Taken together, this study has: (i) identified that miR-33b targets $M Y C$; (ii) suggested that miR-33b loss may contribute to c-Myc over-expression in some medulloblastomas; (iii) revealed that lovastatin is an attractive chemotherapeutic agent against medulloblastoma with a native miR-33b gene; (iv) shown that the resistance of lovastatin in HeLa cells is likely a result of epigenetic inactivation of the $S R E B F 1 \sim m i R-33 b$ gene; and (v) presented 
a highly promising therapeutic option, using drug repurposing and a miRNA as a biomarker, against cancers that over-express c-Myc. 


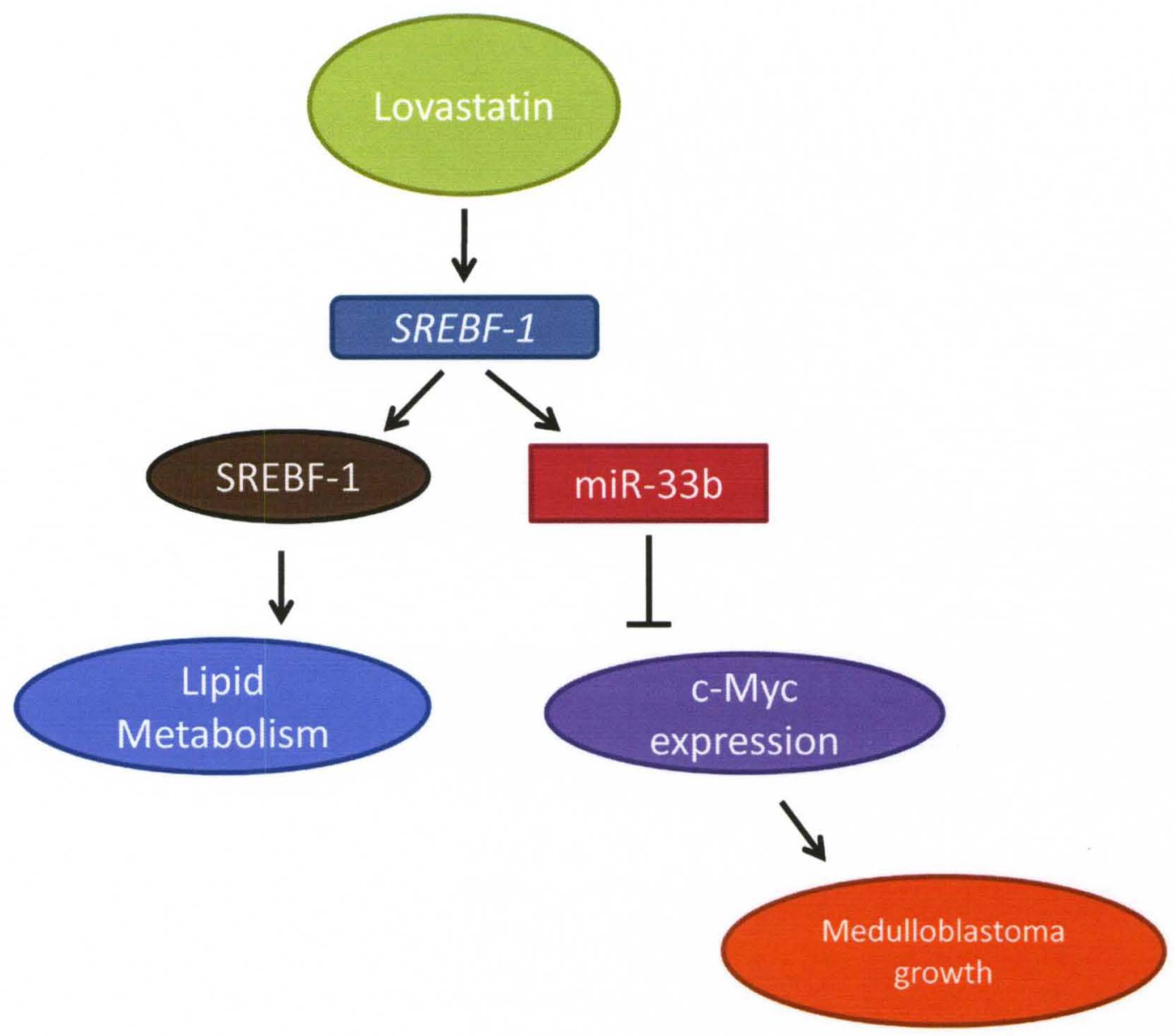

Fig. 35: Proposed schematic of lovastatin induce inhibition of medulloblastoma cell growth. Lovastatin decreases medulloblastoma cell growth by positively modulating miR-33b expression. 


\section{REFERENCES}

1. McKinney PA (2005) Central nervous system tumours in children: Epidemiology and risk factors. Bioelectromagnetics 26(S7):S60-S68.

2. Pritchard-Jones K, Kaatsch P, Steliarova-Foucher E, Stiller CA, \& Coebergh JWW (2006) Cancer in children and adolescents in Europe: Developments over 20 years and future challenges. European Journal of Cancer 42(13):2183-2190.

3. Abdullah S, Qaddoumi I, \& Bouffet E (2008) Advances in the Management of Pediatric Central Nervous System Tumors. Annals of the New York Academy of Sciences 1138(1):22-31.

4. Gottardo NG \& Gajjar A (2008) Chemotherapy for Malignant Brain Tumors of Childhood. Journal of Child Neurology 23(10):1149-1159.

5. Packer RJ (2008) Childhood Brain Tumors: Accomplishments and Ongoing Challenges. Journal of Child Neurology 23(10):1122-1127.

6. Louis D, et al. (2007) The 2007 WHO Classification of Tumours of the Central Nervous System. Acta Neuropathologica 114(2):97-109.

7. Lannering B, et al. (2009) Classification, incidence and survival analyses of children with CNS tumours diagnosed in Sweden 1984-2005. Acta Pædiatrica 98(10):1620-1627.

8. VÃ@zina L-G (2008) Imaging of Central Nervous System Tumors in Children: Advances and Limitations. Journal of Child Neurology 23(10):1128-1135.

9. Mueller S \& Chang S (2009) Pediatric Brain Tumors: Current Treatment Strategies and Future Therapeutic Approaches. Neurotherapeutics 6(3):570-586.

10. Mulhern RK, Merchant TE, Gajjar A, Reddick WE, \& Kun LE (2004) Late neurocognitive sequelae in survivors of brain tumours in childhood. (Translated from eng) Lancet Oncol 5(7):399-408 (in eng).

11. Mulhern RK, et al. (2004) Attentional functioning and white matter integrity among survivors of malignant brain tumors of childhood. (Translated from eng) $\mathrm{J} / \mathrm{nt}$ Neuropsychol Soc 10(2):180-189 (in eng).

12. Laughton SJ, et al. (2008) Endocrine Outcomes for Children With Embryonal Brain Tumors After Risk-Adapted Craniospinal and Conformal Primary-Site Irradiation and High-Dose Chemotherapy With Stem-Cell Rescue on the SJMB-96 Trial. Journal of Clinical Oncology 26(7):1112-1118.

13. Dhall G (2009) Medulloblastoma. Journal of Child Neurology 24(11):1418-1430.

14. Chan AW, et al. (2000) Adult Medulloblastoma: Prognostic Factors and Patterns of Relapse. Neurosurgery 47(3):623-632.

15. Rorke LB (1983) The cerebellar medulloblastoma and its relationship to primitive neuroectodermal tumors. (Translated from eng) J Neuropathol Exp Neuro/ 42(1):1-15 (in eng).

16. Pomeroy SL, et al. (2002) Prediction of central nervous system embryonal tumour outcome based on gene expression. Nature 415(6870):436-442.

17. Fan X \& Eberhart CG (2008) Medulloblastoma Stem Cells. Journal of Clinical Oncology 26(17):2821-2827. 
18. Eberhart CG \& Burger PC (2003) Anaplasia and grading in medulloblastomas. (Translated from eng) Brain Pathol 13(3):376-385 (in eng).

19. Gorlin RJ (1987) Nevoid basal-cell carcinoma syndrome. (Translated from eng) Medicine (Baltimore) 66(2):98-113 (in eng).

20. Hamilton SR, et al. (1995) The Molecular Basis of Turcot's Syndrome. New England Journal of Medicine 332(13):839-847.

21. Polkinghorn WR \& Tarbell NJ (2007) Medulloblastoma: tumorigenesis, current clinical paradigm, and efforts to improve risk stratification. Nat Clin Prac Oncol 4(5):295-304.

22. Gilbertson RJ \& Ellison DW (2008) The Origins of Medulloblastoma Subtypes. Annual Review of Pathology: Mechanisms of Disease 3(1):341-365.

23. Logan CY \& Nusse R (2004) THE WNT SIGNALING PATHWAY IN DEVELOPMENT AND DISEASE. Annual Review of Cell and Developmental Biology 20(1):781-810.

24. Reya T \& Clevers $\mathrm{H}$ (2005) Wnt signalling in stem cells and cancer. Nature 434(7035):843-850.

25. Barker N \& Clevers H (2006) Mining the Wnt pathway for cancer therapeutics. Nat Rev Drug Discov 5(12):997-1014.

26. Chen G \& Courey AJ (2000) Groucho/TLE family proteins and transcriptional repression. Gene 249(1-2):1-16.

27. Ellison DW, et al. (2005) Ã $\ddot{Y}$-Catenin Status Predicts a Favorable Outcome in Childhood Medulloblastoma: The United Kingdom Children's Cancer Study Group Brain Tumour Committee. Journal of Clinical Oncology 23(31):7951-7957.

28. Dahmen RP, et al. (2001) Deletions of AXIN1, a Component of the WNT/wingless Pathway, in Sporadic Medulloblastomas. Cancer Research 61(19):7039-7043.

29. EBERHART CG, TIHAN T, \& BURGER PC (2000) Nuclear Localization and Mutation of [beta]-Catenin in Medulloblastomas. Journal of Neuropathology \& Experimental Neurology 59(4):333-337.

30. Huang H, et al. (2000) APC Mutations in Sporadic Medulloblastomas. Am J Pathol 156(2):433-437.

31. Kool M, et al. (2008) Integrated Genomics Identifies Five Medulloblastoma Subtypes with Distinct Genetic Profiles, Pathway Signatures and Clinicopathological Features. PLOS ONE 3(8):e3088.

32. Thompson MC, et al. (2006) Genomics Identifies Medulloblastoma Subgroups That Are Enriched for Specific Genetic Alterations. Journal of Clinical Oncology 24(12):1924-1931.

33. Varjosalo M \& Taipale J (2008) Hedgehog: functions and mechanisms. Genes \& Development 22(18):2454-2472.

34. BeachyPhilip A, KarhadkarSunil S, \& BermanDavid M (2004) Tissue repair and stem cell renewal in carcinogenesis. Nature 432(7015):324-331.

35. Hahn H, et al. (1996) A Mammalian patched Homolog is Expressed in Target Tissues of sonic hedgehog and Maps to a Region Associated with Developmental Abnormalities. Journal of Biological Chemistry 271(21):12125-12128.

36. Bottaro DP, et al. (1991) Identification of the hepatocyte growth factor receptor as the c-met proto-oncogene product. (Translated from eng) Science 251(4995):802-804 (in eng).

37. Weidner KM, et al. (1996) Interaction between Gab1 and the c-Met receptor tyrosine kinase is responsible for epithelial morphogenesis. Nature 384(6605):173-176.

38. Li Y, et al. (2005) The Scatter Factor/Hepatocyte Growth Factor: c-Met Pathway in Human Embryonal Central Nervous System Tumor Malignancy. Cancer Research 65(20):9355-9362. 
39. Li Y, et al. (2007) Functional and molecular interactions between the HGF/c-Met pathway and c-Myc in large-cell medulloblastoma. Lab Invest 88(2):98-111.

40. Grotzer MA, et al. (2000) TrkC Expression Predicts Good Clinical Outcome in Primitive Neuroectodermal Brain Tumors. Journal of Clinical Oncology 18(5):1027.

41. Clifford SC, et al. (2006) Wnt/Wingless pathway activation and chromosome 6 loss characterize a distinct molecular sub-group of medulloblastomas associated with a favorable prognosis. (Translated from eng) Cell Cycle 5(22):2666-2670 (in eng).

42. Sasai K, et al. (2007) Medulloblastomas Derived from Cxcr6 Mutant Mice Respond to Treatment with a Smoothened Inhibitor. Cancer Research 67(8):3871-3877.

43. Gilbertson R \& Ellison D (2008) The origins of medulloblastoma subtypes. Annu Rev Pathol 3:341 - 365.

44. Pfister S, et al. (2009) Outcome Prediction in Pediatric Medulloblastoma Based on DNA Copy-Number Aberrations of Chromosomes $6 q$ and $17 q$ and the MYC and MYCN Loci. Journal of Clinical Oncology 27(10):1627-1636.

45. Grotzer M, et al. (2001) MYC messenger RNA expression predicts survival outcome in childhood primitive neuroectodermal tumor/medulloblastoma. Clin Cancer Res $7(8): 2425$ - 2433.

46. Gajjar A, et al. (2004) Clinical, Histopathologic, and Molecular Markers of Prognosis: Toward a New Disease Risk Stratification System for Medulloblastoma. Journal of Clinical Oncology 22(6):984-993.

47. Sheiness D \& Bishop JM (1979) DNA and RNA from Uninfected Vertebrate Cells Contain Nucleotide Sequences Related to the Putative Transforming Gene of Avian Myelocytomatosis Virus. J. Virol. 31(2):514-521.

48. Vennstrom B, Sheiness D, Zabielski J, \& Bishop JM (1982) Isolation and characterization of $c-m y c$, a cellular homolog of the oncogene $(\mathrm{v}-\mathrm{myc})$ of avian myelocytomatosis virus strain 29. J. Virol. 42(3):773-779.

49. Gregory MA \& Hann SR (2000) c-Myc Proteolysis by the Ubiquitin-Proteasome Pathway: Stabilization of c-Myc in Burkitt's Lymphoma Cells. Mol. Cell. Biol. 20(7):2423-2435.

50. Meyer N \& Penn LZ (2008) Reflecting on 25 years with MYC. Nat Rev Cancer 8(12):976990.

51. Oster SK, Ho CS, Soucie EL, \& Penn LZ (2002) The myc oncogene: MarvelouslY Complex. (Translated from eng) Adv Cancer Res 84:81-154 (in eng).

52. Vita $M \&$ Henriksson $M$ (2006) The Myc oncoprotein as a therapeutic target for human cancer. Seminars in Cancer Biology 16(4):318-330.

53. Conzen SD, et al. (2000) Induction of Cell Cycle Progression and Acceleration of Apoptosis Are Two Separable Functions of c-Myc: Transrepression Correlates with Acceleration of Apoptosis. Mol. Cell. Biol. 20(16):6008-6018.

54. Henriksson M, Bakardjiev A, Klein G, \& Luscher B (1993) Phosphorylation sites mapping in the $\mathrm{N}$-terminal domain of c-myc modulate its transforming potential. (Translated from eng) Oncogene 8(12):3199-3209 (in eng).

55. Herbst A, et al. (2005) A conserved element in Myc that negatively regulates its proapoptotic activity. (Translated from eng) EMBO Rep 6(2):177-183 (in eng).

56. Ponzielli R, Katz S, Barsyte-Lovejoy D, \& Penn LZ (2005) Cancer therapeutics: Targeting the dark side of Myc. European Journal of Cancer 41(16):2485-2501.

57. de Alboran IM, et al. (2001) Analysis of C-MYC Function in Normal Cells via Conditional Gene-Targeted Mutation. Immunity 14(1):45-55.

58. Trumpp A, et al. (2001) c-Myc regulates mammalian body size by controlling cell number but not cell size. Nature 414(6865):768-773. 
59. Perez-Roger I, Kim S-H, Griffiths B, Sewing A, \& Land H (1999) Cyclins D1 and D2 mediate Myc-induced proliferation via sequestration of p27Kip1 and p21Cip1. EMBO J 18(19):5310-5320.

60. Perez-Roger I, Solomon DL, Sewing A, \& Land H (1997) Myc activation of cyclin E/Cdk2 kinase involves induction of cyclin E gene transcription and inhibition of p27(Kip1) binding to newly formed complexes. (Translated from eng) Oncogene 14(20):2373-2381 (in eng).

61. Ponzielli R, Katz S, Barsyte-Lovejoy D, \& Penn L (2005) Cancer therapeutics: targeting the dark side of Myc. Eur J Cancer 41(16):2485 - 2501.

62. Meyskens FL, Jr. \& Gerner EW (1999) Development of difluoromethylornithine (DFMO) as a chemoprevention agent. (Translated from eng) Clin Cancer Res 5(5):945-951 (in eng).

63. Welcker M \& Clurman BE (2008) FBW7 ubiquitin ligase: a tumour suppressor at the crossroads of cell division, growth and differentiation. Nat Rev Cancer 8(2):83-93.

64. Kubota S, Kiyosawa H, Nomura Y, Yamada T, \& Seyama Y (1997) Ornithine Decarboxylase Overexpression in Mouse 10T12 Fibroblasts: Cellular Transformation and Invasion. Journal of the National Cancer Institute 89(8):567-571.

65. Rottmann S \& Luscher B (2006) The Mad side of the Max network: antagonizing the function of Myc and more. (Translated from eng) Curr Top Microbiol Immunol 302:63122 (in eng).

66. Iritani BM \& Eisenman RN (1999) C-Myc enhances protein synthesis and cell size during B lymphocyte development. (Translated from eng) Proc Natl Acad Sci U S A 96(23):13180-13185 (in eng).

67. Dang CV, et al. (2006) The c-Myc target gene network. Seminars in Cancer Biology 16(4):253-264.

68. Dang CV (1999) c-Myc Target Genes Involved in Cell Growth, Apoptosis, and Metabolism. Mol. Cell. Biol. 19(1):1-11.

69. Amundson SA, et al. (1999) Fluorescent CDNA microarray hybridization reveals complexity and heterogeneity of cellular genotoxic stress responses. (Translated from eng) Oncogene 18(24):3666-3672 (in eng).

70. Marhin WW, Chen S, Facchini LM, Fornace AJ, Jr., \& Penn LZ (1997) Myc represses the growth arrest gene gadd45. (Translated from eng) Oncogene 14(23):2825-2834 (in eng).

71. Mai S, Hanley-Hyde J, \& Fluri M (1996) C-Myc overexpression associated DHFR gene amplification in hamster, rat, mouse and human cell lines. (Translated from eng) Oncogene 12(2):277-288 (in eng).

72. Li Q \& Dang CV (1999) c-Myc Overexpression Uncouples DNA Replication from Mitosis. Mol. Cell. Biol. 19(8):5339-5351.

73. Yin XY, Grove L, Datta NS, Long MW, \& Prochownik EV (1999) C-myc overexpression and p53 loss cooperate to promote genomic instability. (Translated from eng) Oncogene 18(5):1177-1184 (in eng).

74. Prochownik EV \& Li Y (2007) The ever expanding role for C-Myc in promoting genomic instability. (Translated from eng) Cell Cycle 6(9):1024-1029 (in eng).

75. Louis SF, et al. (2005) c-Myc induces chromosomal rearrangements through telomere and chromosome remodeling in the interphase nucleus. (Translated from eng) Proc Nat/ Acad Sci U S A 102(27):9613-9618 (in eng).

76. Gorrini C, et al. (2007) Tip60 is a haplo-insufficient tumour suppressor required for an oncogene-induced DNA damage response. Nature 448(7157):1063-1067. 
77. Dews $M$, et al. (2006) Augmentation of tumor angiogenesis by a Myc-activated microRNA cluster. Nat Genet 38(9):1060-1065.

78. Delaloy $C$, et al. (MicroRNA-9 Coordinates Proliferation and Migration of Human Embryonic Stem Cell-Derived Neural Progenitors. Cell Stem Cell 6(4):323-335.

79. Uchida N (MicroRNA-9 Controls a Migratory Mechanism in Human Neural Progenitor Cells. Cell Stem Cell 6(4):294-296.

80. Ma L, et al. (miR-9, a MYC/MYCN-activated microRNA, regulates E-cadherin and cancer metastasis. Nat Cell Biol 12(3):247-256.

81. Hoang AT, Cohen KJ, Barrett JF, Bergstrom DA, \& Dang CV (1994) Participation of cyclin A in Myc-induced apoptosis. (Translated from eng) Proc Nat/ Acad Sci U S A 91(15):68756879 (in eng).

82. Albihn A, Lovén J, Ohlsson J, Osorio LM, \& Henriksson M (2006) C-Myc-dependent etoposide-induced apoptosis involves activation of Bax and caspases, and PKCdelta signaling. Journal of Cellular Biochemistry 98(6):1597-1614.

83. Brunelle JK, et al. (2004) c-Myc sensitization to oxygen deprivation-induced cell death is dependent on Bax/Bak, but is independent of p53 and hypoxia-inducible factor-1. (Translated from eng) J Biol Chem 279(6):4305-4312 (in eng).

84. Teitz T, et al. (2000) Caspase 8 is deleted or silenced preferentially in childhood neuroblastomas with amplification of MYCN. (Translated from eng) Nat Med 6(5):529535 (in eng).

85. Wagner AJ, Kokontis JM, \& Hay N (1994) Myc-mediated apoptosis requires wild-type p53 in a manner independent of cell cycle arrest and the ability of p53 to induce p21waf1/cip1. (Translated from eng) Genes Dev 8(23):2817-2830 (in eng).

86. Pelengaris $S \&$ Khan $M(2003)$ The $c-M Y C$ oncoprotein as a treatment target in cancer and other disorders of cell growth. (Translated from eng) Expert Opin Ther Targets 7(5):623-642 (in eng).

87. Knoepfler PS, Cheng PF, \& Eisenman RN (2002) N-myc is essential during neurogenesis for the rapid expansion of progenitor cell populations and the inhibition of neuronal differentiation. (Translated from eng) Genes Dev 16(20):2699-2712 (in eng).

88. Wilson A, et al. (2004) c-Myc controls the balance between hematopoietic stem cell selfrenewal and differentiation. Genes \& Development 18(22):2747-2763.

89. Laurenti E, et al. (2008) Hematopoietic Stem Cell Function and Survival Depend on cMyc and N-Myc Activity. Cell Stem Cell 3(6):611-624.

90. Cartwright $\mathrm{P}$, et al. (2005) LIF/STAT3 controls ES cell self-renewal and pluripotency by a Myc-dependent mechanism. Development 132(5):885-896.

91. Okita K, Ichisaka T, \& Yamanaka S (2007) Generation of germline-competent induced pluripotent stem cells. Nature 448(7151):313-317.

92. Nakagawa M, et al. (2008) Generation of induced pluripotent stem cells without Myc from mouse and human fibroblasts. Nat Biotech 26(1):101-106.

93. Singh AM \& Dalton S (2009) The Cell Cycle and Myc Intersect with Mechanisms that Regulate Pluripotency and Reprogramming. Cell Stem Cell 5(2):141-149.

94. Aldosari N, et al. (2002) MYCC and MYCN Oncogene Amplification in Medulloblastoma. Archives of Pathology \& Laboratory Medicine 126(5):540-544.

95. Eberhart C, et al. (2004) Histopathological and molecular prognostic markers in medulloblastoma: c-myc, N-myc, TrkC, and anaplasia. J Neuropathol Exp Neurol 63(5):441 - 449.

96. Stearns D, et al. (2006) C-Myc Overexpression Causes Anaplasia in Medulloblastoma. Cancer Research 66(2):673-681. 
97. Rao G, Pedone CA, Coffin CM, Holland EC, \& Fults DW (2003) C-Myc enhances sonic hedgehog-induced medulloblastoma formation from nestin-expressing neural progenitors in mice. (Translated from eng) Neoplasia 5(3):198-204 (in eng).

98. Browd SR, et al. (2006) N-myc Can Substitute for Insulin-Like Growth Factor Signaling in a Mouse Model of Sonic Hedgehogâ€"Induced Medulloblastoma. Cancer Research 66(5):2666-2672.

99. Herms J, et al. (2000) c-MYC expression in medulloblastoma and its prognostic value. International Journal of Cancer 89(5):395-402.

100. Stearns D, et al. (2006) c-myc overexpression causes anaplasia in medulloblastoma. (Translated from eng) Cancer Res 66(2):673-681 (in eng).

101. Taub R, et al. (1984) A novel alteration in the structure of an activated c-myc gene in a variant $\mathrm{t}(2 ; 8)$ burkitt lymphoma. Cell $37(2): 511-520$.

102. Paulin FE, Chappell SA, \& Willis AE (1998) A single nucleotide change in the c-myc internal ribosome entry segment leads to enhanced binding of a group of protein factors. (Translated from eng) Nucleic Acids Res 26(13):3097-3103 (in eng).

103. Peng $H$, Diss TIM, Isaacson PG, \& Pan L (1997) c-myc GENE ABNORMALITIES IN MUCOSAASSOCIATED LYMPHOID TISSUE (MALT) LYMPHOMAS. The Journal of Pathology 181(4):381-386.

104. He TC, et al. (1998) Identification of C-MYC as a target of the APC pathway. (Translated from eng) Science 281(5382):1509-1512 (in eng).

105. Yeh $\mathrm{E}$, et al. (2004) A signalling pathway controlling c-Myc degradation that impacts oncogenic transformation of human cells. Nat Cell Biol 6(4):308-318.

106. Adhikary $S$ \& Eilers $M(2005)$ Transcriptional regulation and transformation by Myc proteins. Nat Rev Mol Cell Biol 6(8):635-645.

107. Kim SY, Herbst A, Tworkowski KA, Salghetti SE, \& Tansey WP (2003) Skp2 Regulates Myc Protein Stability and Activity. Molecular Cell 11(5):1177-1188.

108. Sears R, et al. (2000) Multiple Ras-dependent phosphorylation pathways regulate Myc protein stability. Genes \& Development 14(19):2501-2514.

109. Friedman RC, Farh KK-H, Burge CB, \& Bartel DP (2009) Most mammalian mRNAs are conserved targets of microRNAs. Genome Research 19(1):92-105.

110. Denli AM, Tops BBJ, Plasterk RHA, Ketting RF, \& Hannon GJ (2004) Processing of primary microRNAs by the Microprocessor complex. Nature 432(7014):231-235.

111. Meister G, et al. (2004) Human Argonaute2 Mediates RNA Cleavage Targeted by miRNAs and siRNAs. Molecular Cell 15(2):185-197.

112. Gregory RI, Chendrimada TP, Cooch N, \& Shiekhattar R (2005) Human RISC Couples MicroRNA Biogenesis and Posttranscriptional Gene Silencing. Cell 123(4):631-640.

113. Bartel D (2004) MicroRNAs: genomics, biogenesis, mechanism, and function. Cell 116:281 - 297.

114. Kahvejian A, Svitkin YV, Sukarieh R, M'Boutchou MN, \& Sonenberg N (2005) Mammalian poly(A)-binding protein is a eukaryotic translation initiation factor, which acts via multiple mechanisms. (Translated from eng) Genes Dev 19(1):104-113 (in eng).

115. Eulalio A, et al. (2009) The RRM domain in GW182 proteins contributes to miRNAmediated gene silencing. (Translated from eng) Nucleic Acids Res 37(9):2974-2983 (in eng).

116. Calin G, et al. (2004) MicroRNA profiling reveals distinct signatures in B cell chronic lymphocytic leukemias. Proc Natl Acad Sci USA 101:11755 - 11760. 
117. Selcuklu SD, Yakicier MC, \& Erson AE (2009) An investigation of microRNAs mapping to breast cancer related genomic gain and loss regions. Cancer Genetics and Cytogenetics 189(1):15-23.

118. Kumar MS, Lu J, Mercer KL, Golub TR, \& Jacks T (2007) Impaired microRNA processing enhances cellular transformation and tumorigenesis. Nat Genet 39(5):673-677.

119. O'Donnell KA, Wentzel EA, Zeller KI, Dang CV, \& Mendell JT (2005) c-Myc-regulated microRNAs modulate E2F1 expression. Nature 435(7043):839-843.

120. He L, et al. (2005) A microRNA polycistron as a potential human oncogene. Nature 435(7043):828-833.

121. Eis PS, et al. (2005) Accumulation of miR-155 and BIC RNA in human B cell lymphomas. Proceedings of the National Academy of Sciences of the United States of America 102(10):3627-3632.

122. Johnson SM, et al. (2005) RAS is Regulated by the let-7 MicroRNA Family. Cell 120(5):635-647.

123. Mayr C, Hemann MT, \& Bartel DP (2007) Disrupting the Pairing Between let-7 and Hmga2 Enhances Oncogenic Transformation. Science 315(5818):1576-1579.

124. Esquela-Kerscher $A$, et al. (2008) The $<i>\mid e t-7</ i>$ microRNA reduces tumor growth in mouse models of lung cancer. Cell Cycle 7(6):759-764.

125. Childs G, et al. (2009) Low-Level Expression of MicroRNAs let-7d and miR-205 Are Prognostic Markers of Head and Neck Squamous Cell Carcinoma. The American Journal of Pathology 174(3):736-745.

126. Boyerinas B, Park S-M, Hau A, Murmann AE, \& Peter ME (The role of let-7 in cell differentiation and cancer. Endocrine-Related Cancer 17(1):F19-F36.

127. Talotta F, et al. (2008) An autoregulatory loop mediated by miR-21 and PDCD4 controls the AP-1 activity in RAS transformation. Oncogene 28(1):73-84.

128. MaX, et al. (Loss of the miR-21 allele elevates the expression of its target genes and reduces tumorigenesis. Proceedings of the National Academy of Sciences 108(25):1014410149.

129. Hatley ME, et al. (Modulation of K-Ras-Dependent Lung Tumorigenesis by MicroRNA-21. Cancer Cell 18(3):282-293.

130. Malumbres M \& Barbacid M (2001) Milestones in cell division : To cycle or not to cycle: a critical decision in cancer. Nat Rev Cancer 1(3):222-231.

131. Emmrich S \& PÃ $1 / 4$ tzer BM (Checks and balances: E2Fâ $€$ "microRNA crosstalk in cancer control. Cell Cycle 9(13):2553-2565.

132. Petrocca F, Vecchione A, \& Croce CM (2008) Emerging Role of miR-106b-25/miR-17-92 Clusters in the Control of Transforming Growth Factor $\hat{i}^{2}$ Signaling. Cancer Research 68(20):8191-8194.

133. Sylvestre $Y$, et al. (2007) An E2F/miR-20a Autoregulatory Feedback Loop. Journal of Biological Chemistry 282(4):2135-2143.

134. Liu $Q$ et al. (2008) miR-16 family induces cell cycle arrest by regulating multiple cell cycle genes. Nucleic Acids Research 36(16):5391-5404.

135. Takeshita F, et al. (2009) Systemic Delivery of Synthetic MicroRNA-16 Inhibits the Growth of Metastatic Prostate Tumors via Downregulation of Multiple Cell-cycle Genes. Mol Ther 18(1):181-187.

136. Pierson J, Hostager B, Fan R, \& Vibhakar R (2008) Regulation of cyclin dependent kinase 6 by microRNA 124 in medulloblastoma. Journal of Neuro-Oncology 90(1):1-7. 
137. Kozaki K-i, Imoto I, Mogi S, Omura K, \& Inazawa J (2008) Exploration of TumorSuppressive MicroRNAs Silenced by DNA Hypermethylation in Oral Cancer. Cancer Research 68(7):2094-2105.

138. Lujambio A, et al. (2007) Genetic Unmasking of an Epigenetically Silenced microRNA in Human Cancer Cells. Cancer Research 67(4):1424-1429.

139. Lal A, et al. (2008) p16(INK4a) translation suppressed by miR-24. (Translated from eng) PLOS ONE 3(3):e1864 (in eng).

140. Boominathan $L(T h e$ tumor suppressors $p 53, p 63$, and $p 73$ are regulators of microRNA processing complex. (Translated from eng) PLOS ONE 5(5):e10615 (in eng).

141. Chang WY, Andrews J, Carter DE, \& Dagnino L (2006) Differentiation and Injury-Repair Signals Modulate the Interaction of E2F and pRB Proteins with Novel Target Genes in Keratinocytes. Cell Cycle 5(16):1872-1879.

142. Calin GA, et al. (2008) MiR-15a and miR-16-1 cluster functions in human leukemia. Proceedings of the National Academy of Sciences 105(13):5166-5171.

143. Palm W \& de Lange T (2008) How Shelterin Protects Mammalian Telomeres. Annual Review of Genetics 42(1):301-334.

144. Ben-Porath I \& Weinberg RA (2005) The signals and pathways activating cellular senescence. The International Journal of Biochemistry \& Cell Biology 37(5):961-976.

145. Kumamoto K, et al. (2008) Nutlin-3a Activates p53 to Both Down-regulate Inhibitor of Growth 2 and Up-regulate mir-34a, mir-34b, and mir-34c Expression, and Induce Senescence. Cancer Research 68(9):3193-3203.

146. Tazawa H, Kagawa S, \& Fujiwara T (MicroRNAs as potential target gene in cancer gene therapy of gastrointestinal tumors. Expert Opinion on Biological Therapy 11(2):145-155.

147. Brosh R, et al. (2008) p53-repressed miRNAs are involved with E2F in a feed-forward loop promoting proliferation. Mol Syst Biol 4.

148. Mitomo S, et al. (2008) Downregulation of miR-138 is associated with overexpression of human telomerase reverse transcriptase protein in human anaplastic thyroid carcinoma cell lines. Cancer Science 99(2):280-286.

149. Bonifacio LN \& Jarstfer MB (2010) MiRNA Profile Associated with Replicative Senescence, Extended Cell Culture, and Ectopic Telomerase Expression in Human Foreskin Fibroblasts. (Translated from English) PLOS ONE 5(9) (in English).

150. Yamakuchi $\mathrm{M}$, et al. (P53-induced microRNA-107 inhibits HIF-1 and tumor angiogenesis. Proceedings of the National Academy of Sciences 107(14):6334-6339.

151. Ghosh AK, et al. (2009) Aberrant regulation of $\mathrm{pVHL}$ levels by microRNA promotes the HIF/VEGF axis in CLL B cells. Blood 113(22):5568-5574.

152. Ghosh G, et al. (2010) Hypoxia-induced microRNA-424 expression in human endothelial cells regulates HIF-alpha isoforms and promotes angiogenesis. (Translated from English) Journal of Clinical Investigation 120(11):4141-4154 (in English).

153. Kulshreshtha R, et al. (2007) A MicroRNA Signature of Hypoxia. Mol. Cell. Biol. 27(5):1859-1867.

154. Chen Z, Li Y, Zhang H, Huang P, \& Luthra R (Hypoxia-regulated microRNA-210 modulates mitochondrial function and decreases ISCU and COX10 expression. Oncogene 29(30):4362-4368.

155. Fish JE, et al. (2008) miR-126 Regulates Angiogenic Signaling and Vascular Integrity. Developmental Cell 15(2):272-284.

156. Hua Z, et al. (2006) MiRNA-directed regulation of VEGF and other angiogenic factors under hypoxia. (Translated from eng) PLOS ONE 1:e116 (in eng). 


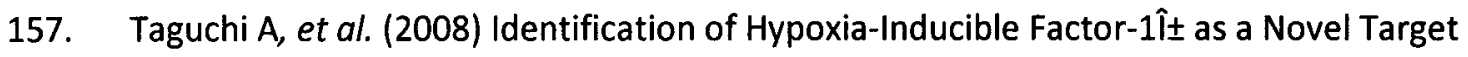
for miR-17-92 MicroRNA Cluster. Cancer Research 68(14):5540-5545.

158. Dykxhoorn DM, et al. (2009) miR-200 enhances mouse breast cancer cell colonization to form distant metastases. (Translated from eng) PLOS ONE 4(9):e7181 (in eng).

159. Hurteau GJ, Carlson JA, Roos E, \& Brock GJ (2009) Stable expression of miR-200c alone is sufficient to regulate TCF8 (ZEB1) and restore E-cadherin expression. Cell Cycle 8(13):2064-2069.

160. Ma $\mathrm{L}$, et al. (Therapeutic silencing of miR-10b inhibits metastasis in a mouse mammary tumor model. Nat Biotech 28(4):341-347.

161. Zhu S, et al. (MicroRNA-21 targets tumor suppressor genes in invasion and metastasis. Cell Res 18(3):350-359.

162. Zhu S, Si M-L, Wu H, \& Mo Y-Y (2007) MicroRNA-21 Targets the Tumor Suppressor Gene Tropomyosin 1 (TPM1). Journal of Biological Chemistry 282(19):14328-14336.

163. Gabriely G, et al. (2008) MicroRNA 21 Promotes Glioma Invasion by Targeting Matrix Metalloproteinase Regulators. Mol. Cell. Biol. 28(17):5369-5380.

164. Meng F, et al. (2007) MicroRNA-21 Regulates Expression of the PTEN Tumor Suppressor Gene in Human Hepatocellular Cancer. Gastroenterology 133(2):647-658.

165. Kota J, et al. (2009) Therapeutic microRNA Delivery Suppresses Tumorigenesis in a Murine Liver Cancer Model. Cell 137(6):1005-1017.

166. Krutzfeldt J, et al. (2005) Silencing of microRNAs in vivo with /'antagomirs/'. Nature 438(7068):685-689.

167. KrÃ $1 / 4$ tzfeldt J, et al. (2007) Specificity, duplex degradation and subcellular localization of antagomirs. Nucleic Acids Research 35(9):2885-2892.

168. Fontana $L$, et al. (2008) Antagomir-17-5p abolishes the growth of therapy-resistant neuroblastoma through p21 and BIM. (Translated from eng) PLOS ONE 3(5):e2236 (in eng).

169. ElmÃ@On J, et al. (2008) Antagonism of microRNA-122 in mice by systemically administered LNA-antimiR leads to up-regulation of a large set of predicted target mRNAs in the liver. Nucleic Acids Research 36(4):1153-1162.

170. Goodrich LV, Milenkovï̈ł L, Higgins KM, \& Scott MP (1997) Altered Neural Cell Fates and Medulloblastoma in Mouse patched Mutants. Science 277(5329):1109-1113.

171. Garzia L, et al. (2009) MicroRNA-199b-5p Impairs Cancer Stem Cells through Negative Regulation of HES1 in Medulloblastoma. PLOS ONE 4(3):e4998.

172. Ferretti E, et al. (2009) MicroRNA profiling in human medulloblastoma. International Journal of Cancer 124(3):568-577.

173. Northcott PA, et al. (2009) The miR-17/92 polycistron is up-regulated in sonic hedgehogdriven medulloblastomas and induced by $\mathrm{N}$-myc in sonic hedgehog-treated cerebellar neural precursors. (Translated from eng) Cancer Res 69(8):3249-3255 (in eng).

174. Kenneth NS, et al. (2007) TRRAP and GCN5 are used by c-Myc to activate RNA polymerase III transcription. Proceedings of the National Academy of Sciences 104(38):14917-14922.

175. Schulte JH, et al. (2008) MYCN regulates oncogenic MicroRNAs in neuroblastoma. International Journal of Cancer 122(3):699-704.

176. Chang T-C, et al. (2008) Widespread microRNA repression by Myc contributes to tumorigenesis. Nat Genet 40(1):43-50.

177. Calin GA, et al. (2002) Frequent deletions and down-regulation of micro- RNA genes miR15 and miR16 at 13q14 in chronic lymphocytic leukemia. Proceedings of the National Academy of Sciences 99(24):15524-15529. 
178. Cimmino A, et al. (2005) miR-15 and miR-16 induce apoptosis by targeting BCL2. Proceedings of the National Academy of Sciences of the United States of America 102(39):13944-13949.

179. Dangi-Garimella S, et al. (2009) Raf kinase inhibitory protein suppresses a metastasis signalling cascade involving LIN28 and let-7. EMBO J 28(4):347-358.

180. Bui TV \& Mendell JT (Myc: Maestro of MicroRNAs. (Translated from Eng) Genes Cancer 1(6):568-575 (in Eng).

181. Jensen NA, et al. (2003) Astroglial c-Myc Overexpression Predisposes Mice to Primary Malignant Gliomas. J. Biol. Chem. 278(10):8300-8308.

182. Shi H, et al. (2009) Suppression of N-Myc Downstream-Regulated Gene 2 Is Associated with Induction of Myc in Colorectal Cancer and Correlates Closely with Differentiation. Biological \& Pharmaceutical Bulletin 32(6):968-975.

183. Couillard $M \&$ Trudel $M(2009)<1>C$-myc $</ 1>$ as a modulator of renal stem/progenitor cell population. Developmental Dynamics 238(2):405-414.

184. Shachaf CM, et al. (2004) MYC inactivation uncovers pluripotent differentiation and tumour dormancy in hepatocellular cancer. Nature 431(7012):1112-1117.

185. Amati B, Frank SR, Donjerkovic D, \& Taubert S (2001) Function of the C-Myc oncoprotein in chromatin remodeling and transcription. Biochimica et Biophysica Acta (BBA) Reviews on Cancer 1471(3):M135-M145.

186. Frank SR, Schroeder M, Fernandez P, Taubert S, \& Amati B (2001) Binding of C-Myc to chromatin mediates mitogen-induced acetylation of histone $\mathrm{H} 4$ and gene activation. Genes \& Development 15(16):2069-2082.

187. Stearns D, et al. (2006) c-Myc Overexpression Causes Anaplasia in Medulloblastoma. Cancer Res 66(2):673-681.

188. Gilhuis HJ, et al. (2000) Comparative genomic hybridization of medulloblastomas and clinical relevance: eleven new cases and a review of the literature. Clinical Neurology and Neurosurgery 102(4):203-209.

189. Hambardzumyan D, et al. (2008) PI3K pathway regulates survival of cancer stem cells residing in the perivascular niche following radiation in medulloblastoma in vivo. Genes \& Development 22(4):436-448.

190. Schüller U, et al. (2008) Acquisition of Granule Neuron Precursor Identity Is a Critical Determinant of Progenitor Cell Competence to Form Shh-Induced Medulloblastoma. Cancer Cell 14(2):123-134.

191. Yang Z-J, et al. (2008) Medulloblastoma Can Be Initiated by Deletion of Patched in Lineage-Restricted Progenitors or Stem Cells. Cancer Cell 14(2):135-145.

192. Sampson VB, et al. (2007) MicroRNA Let-7a Down-regulates MYC and Reverts MYCInduced Growth in Burkitt Lymphoma Cells. Cancer Research 67(20):9762-9770.

193. Frenzel A, LovÃ@n J, \& Henriksson MA (Targeting MYC-Regulated miRNAs to Combat Cancer. Genes \& Cancer 1(6):660-667.

194. Kovesdi I, Reichel R, \& Nevins JR (1987) Role of an adenovirus E2 promoter binding factor in E1A-mediated coordinate gene control. Proceedings of the National Academy of Sciences 84(8):2180-2184.

195. DeGregori J, Kowalik T, \& Nevins J (1995) Cellular targets for activation by the E2F1 transcription factor include DNA synthesis- and G1/S-regulatory genes [published erratum appears in Mol Cell Biol 1995 Oct;15(10):5846-7]. Mol. Cell. Biol. 15(8):42154224. 
196. Helin K \& Harlow E (1994) Heterodimerization of the transcription factors E2F-1 and DP1 is required for binding to the adenovirus E4 (ORF6/7) protein. J. Virol. 68(8):50275035.

197. Sears R, Ohtani K, \& Nevins J (1997) Identification of positively and negatively acting elements regulating expression of the E2F2 gene in response to cell growth signals. Mol. Cell. Biol. 17(9):5227-5235.

198. Chen C, et al. (Real-time quantification of microRNAs by stemâ€"loop RTâ€"PCR. Nucleic Acids Research 33(20):e179.

199. Lu Z, et al. (2010) miR-301a as an NF-KB activator in pancreatic cancer cells. EMBO J PMID: 21113131.

200. Zhang Z, et al. (2009) MicroRNA miR-210 modulates cellular response to hypoxia through the MYC antagonist MNT. (Translated from eng) Cell Cycle 8(17):2756-2768 (in eng).

201. Griffiths-Jones S, Grocock R, van Dongen S, Bateman A, \& Enright A (2006) miRBase: microRNA sequences, targets and gene nomenclature. Nucleic Acids Res 34:D140 - 144.

202. Mateyak M, Obaya A, Adachi S, \& Sedivy J (1997) Phenotypes of c-Myc-deficient rat fibroblasts isolated by targeted homologous recombination. Cell Growth Differ 8(10):1039-1048.

203. Siu I-M, Lal A, Blankenship JR, Aldosari N, \& Riggins GJ (2003) c-Myc Promoter Activation in Medulloblastoma. Cancer Research 63(16):4773-4776.

204. Ma L, et al. (2010) miR-9, a MYC/MYCN-activated microRNA, regulates E-cadherin and cancer metastasis. Nat Cell Biol 12(3):247-256.

205. Varlakhanova NV, et al. (myc maintains embryonic stem cell pluripotency and selfrenewal. (Translated from eng) Differentiation 80(1):9-19 (in eng).

206. Annabi B, et al. (2008) Tumor environment dictates medulloblastoma cancer stem cell expression and invasive phenotype. (Translated from eng) Mol Cancer Res 6(6):907-916 (in eng).

207. Hemmati HD, et al. (2003) Cancerous stem cells can arise from pediatric brain tumors. (Translated from eng) Proc Natl Acad Sci U S A 100(25):15178-15183 (in eng).

208. Sakakibara S, et al. (2002) RNA-binding protein Musashi family: roles for CNS stem cells and a subpopulation of ependymal cells revealed by targeted disruption and antisense ablation. (Translated from eng) Proc Natl Acad Sci U S A 99(23):15194-15199 (in eng).

209. Kaneko Y, et al. (2000) Musashi1: an evolutionally conserved marker for CNS progenitor cells including neural stem cells. (Translated from eng) Dev Neurosci 22(1-2):139-153 (in eng).

210. Sakakibara S, et al. (1996) Mouse-Musashi-1, a neural RNA-binding protein highly enriched in the mammalian CNS stem cell. (Translated from eng) Dev Biol 176(2):230242 (in eng).

211. Teleman AA, Hietakangas V, Sayadian AC, \& Cohen SM (2008) Nutritional Control of Protein Biosynthetic Capacity by Insulin via Myc in Drosophila. Cell Metabolism 7(1):2132.

212. Podar K, et al. (2006) The small-molecule VEGF receptor inhibitor pazopanib (GW786034B) targets both tumor and endothelial cells in multiple myeloma. Proceedings of the National Academy of Sciences 103(51):19478-19483.

213. Bouchard C, Marquardt J, Bras A, Medema RH, \& Eilers M (2004) Myc-induced proliferation and transformation require Akt-mediated phosphorylation of FoxO proteins. (Translated from eng) EMBO J 23(14):2830-2840 (in eng). 
214. Lu Q \& Hong W (2009) Bcl2 enhances c-Myc-mediated MMP-2 expression of vascular smooth muscle cells. Cellular Signalling 21(7):1054-1059.

215. Gatti G, et al. (2009) Myc Prevents Apoptosis and Enhances Endoreduplication Induced by Paclitaxel. PLOS ONE 4(5):e5442.

216. Shachaf $C$, et al. (2004) MYC inactivation uncovers pluripotent differentiation and tumour dormancy in hepatocellular cancer. Nature 431(7012):1112 - 1117.

217. Zhang Z, et al. (2009) MicroRNA miR-210 modulates cellular response to hypoxia through the MYC antagonist MNT. (Translated from Eng) Cell Cycle 8(17) (in Eng).

218. Aguda BD, Kim Y, Piper-Hunter MG, Friedman A, \& Marsh CB (2008) MicroRNA regulation of a cancer network: Consequences of the feedback loops involving miR-1792, E2F, and Myc. Proceedings of the National Academy of Sciences 105(50):1967819683.

219. Kim HH, et al. (2009) HuR recruits let-7/RISC to repress c-Myc expression. Genes \& Development 23(15):1743-1748.

220. PILLAI RS (2005) MicroRNA function: Multiple mechanisms for a tiny RNA? RNA 11(12):1753-1761.

221. Batra SK, McLendon RE, \& Koo JS (1995) Prognostic implications of chromosome 17p deletions in human medulloblastomas. J Neurooncol 24:39-45.

222. Herms J, Neidt I, \& Luscher B (2000) C-MYC expression in medulloblastoma and its prognostic value. Int $J$ Cancer 89:395-402.

223. Eberhart CG, Kratz J, \& Wang Y (2004) Histopathological and molecular prognostic markers in medulloblastoma: c-myc, N-myc, TrkC, and anaplasia. J Neuropathol Exp Neurol 63:441-449.

224. Aldosari N, Bigner SH, \& Burger PC (2002) MYCC and MYCN oncogene amplification in medulloblastoma. A fluorescence in situ hybridization study on paraffin sections from the Children's Oncology Group. Arch Pathol Lab Med 126:540-544.

225. Bigner SH, Friedman HS, \& Vogelstein B (1990) Amplification of the c-myc gene in human medulloblastoma cell lines and xenografts. Cancer Res 50:2347-2350.

226. Batra SK, Rasheed BK, \& Bigner SH (1994) Oncogenes and anti-oncogenes in human central nervous system tumors. Lab Invest 71:621-637.

227. Pan E, et al. (2005) Isochromosome 17q Is a Negative Prognostic Factor in Poor-Risk Childhood Medulloblastoma Patients. Clinical Cancer Research 11(13):4733-4740.

228. Frühwald MC, et al. (2001) Aberrant hypermethylation of the major breakpoint cluster region in $17 \mathrm{p} 11.2$ in medulloblastomas but not supratentorial PNETs. Genes, Chromosomes and Cancer 30(1):38-47.

229. Blazek ER, Foutch JL, \& Maki G (2007) Daoy medulloblastoma cells that express CD133 are radioresistant relative to $\mathrm{CD} 133$ - cells, and the CD133+ sector is enlarged by hypoxia. International Journal of Radiation Oncology*Biology*Physics 67(1):1-5.

230. Jordan CT, Guzman ML, \& Noble M (2006) Cancer Stem Cells. New England Journal of Medicine 355(12):1253-1261.

231. Dean M, Fojo T, \& Bates S (2005) Tumour stem cells and drug resistance. Nat Rev Cancer 5(4):275-284.

232. Kim DH \& Rossi JJ (2007) Strategies for silencing human disease using RNA interference. (Translated from eng) Nat Rev Genet 8(3):173-184 (in eng).

233. Filipowicz W, Jaskiewicz L, Kolb FA, \& Pillai RS (2005) Post-transcriptional gene silencing by siRNAs and miRNAs. (Translated from eng) Curr Opin Struct Biol 15(3):331-341 (in eng). 
234. Doench JG, Petersen CP, \& Sharp PA (2003) siRNAs can function as miRNAs. (Translated from eng) Genes Dev 17(4):438-442 (in eng).

235. Fire A, et al. (1998) Potent and specific genetic interference by double-stranded RNA in Caenorhabditis elegans. (Translated from eng) Nature 391(6669):806-811 (in eng).

236. Esau C, et al. (2004) MicroRNA-143 regulates adipocyte differentiation. (Translated from eng) J Biol Chem 279(50):52361-52365 (in eng).

237. Krutzfeldt J, et al. (2005) Silencing of microRNAs in vivo with /'antagomirs/'. Nature 438:685 - 689.

238. Kovalchuk 0 , et al. (2008) Involvement of microRNA-451 in resistance of the MCF-7 breast cancer cells to chemotherapeutic drug doxorubicin. Molecular Cancer Therapeutics 7(7):2152-2159.

239. Munker R, Liu CG, Taccioli C, Alder $H$, \& Heerema N (MicroRNA profiles of drug-resistant myeloma cell lines. (Translated from eng) Acta Haematol 123(4):201-204 (in eng).

240. Sleigh SH \& Barton CL (Repurposing Strategies for Therapeutics. Pharmaceutical Medicine 24(3):151-159 110.2165/11536770-000000000-000000000.

241. Siu IM, Lal A, Blankenship JR, Aldosari N, \& Riggins GJ (2003) c-Myc promoter activation in medulloblastoma. (Translated from eng) Cancer Res 63(16):4773-4776 (in eng).

242. Poynter JN, et al. (2005) Statins and the Risk of Colorectal Cancer. New England Journal of Medicine 352(21):2184-2192.

243. Demierre M-F, Higgins PDR, Gruber SB, Hawk E, \& Lippman SM (2005) Statins and cancer prevention. Nat Rev Cancer 5(12):930-942.

244. Horie T, et al. (2010) MicroRNA-33 encoded by an intron of sterol regulatory elementbinding protein 2 (Srebp2) regulates HDL in vivo. Proceedings of the National Academy of Sciences 107(40):17321-17326.

245. Marquart TJ, Allen RM, Ory DS, \& Baldán Á (2010) miR-33 links SREBP-2 induction to repression of sterol transporters. Proceedings of the National Academy of Sciences 107(27):12228-12232.

246. Najafi-Shoushtari SH, et al. (2010) MicroRNA-33 and the SREBP Host Genes Cooperate to Control Cholesterol Homeostasis. Science 328(5985):1566-1569.

247. Rayner KJ, et al. (2010) MiR-33 Contributes to the Regulation of Cholesterol Homeostasis. Science 328(5985):1570-1573.

248. Dimitroulakos J, et al. (2001) Differential Sensitivity of Various Pediatric Cancers and Squamous Cell Carcinomas to Lovastatin-induced Apoptosis: Therapeutic Implications. Clinical Cancer Research 7(1):158-167.

249. Botti RE, Triscari J, Pan HY, \& Zayat J (1991) Concentrations of pravastatin and lovastatin in cerebrospinal fluid in healthy subjects. (Translated from eng) Clin Neuropharmacol 14(3):256-261 (in eng).

250. Guillot F, Misslin P, \& Lemaire M (1993) Comparison of fluvastatin and lovastatin bloodbrain barrier transfer using in vitro and in vivo methods. (Translated from eng) $J$ Cardiovasc Pharmacol 21(2):339-346 (in eng).

251. Hengst $L$ \& Reed SI (1996) Translational control of p27Kip1 accumulation during the cell cycle. (Translated from eng) Science 271(5257):1861-1864 (in eng).

252. Albihn A, Johnsen JI, \& Henriksson MA (MYC in oncogenesis and as a target for cancer therapies. (Translated from eng) Adv Cancer Res 107:163-224 (in eng).

253. Tobert JA, et al. (1982) Cholesterol-lowering effect of mevinolin, an inhibitor of 3hydroxy-3-methylglutaryl-coenzyme a reductase, in healthy volunteers. (Translated from eng) J Clin Invest 69(4):913-919 (in eng). 
254. Sumi S, et al. (1992) Inhibition of pancreatic adenocarcinoma cell growth by lovastatin. (Translated from eng) Gastroenterology 103(3):982-989 (in eng).

255. Wang W \& Macaulay RJ (1999) Mevalonate prevents lovastatin-induced apoptosis in medulloblastoma cell lines. (Translated from eng) Can J Neurol Sci 26(4):305-310 (in eng).

256. Macaulay RJB, Wang W, Dimitroulakos J, Becker LE, \& Yeger H (1999) Lovastatin-induced Apoptosis of Human Medulloblastoma Cell Lines in vitro. Journal of Neuro-Oncology 42(1):1-11.

257. Larner J, et al. (1998) A Phase I-II Trial of Lovastatin for Anaplastic Astrocytoma and Glioblastoma Multiforme. American Journal of Clinical Oncology 21(6):579-583.

258. Kim WS, et al. (2001) Phase II Study of High-Dose Lovastatin in Patients with Advanced Gastric Adenocarcinoma. Investigational New Drugs 19(1):81-83.

259. Burnett ME, White EC, Sih S, von Haken MS, \& Cogen PH (1997) Chromosome arm 17p deletion analysis reveals molecular genetic heterogeneity in supratentorial and infratentorial primitive neuroectodermal tumors of the central nervous system. Cancer Genetics and Cytogenetics 97(1):25-31.

260. DeClue JE, Vass WC, Papageorge AG, Lowy DR, \& Willumsen BM (1991) Inhibition of cell growth by lovastatin is independent of ras function. (Translated from eng) Cancer Res 51(2):712-717 (in eng).

261. Goffin J \& Eisenhauer E (2002) DNA methyltransferase inhibitorsâ $€^{\prime \prime}$ state of the art. Annals of Oncology 13(11):1699-1716.

262. Yoo CB \& Jones PA (2006) Epigenetic therapy of cancer: past, present and future. Nat Rev Drug Discov 5(1):37-50.

263. Stefani G \& Slack FJ (2008) Small non-coding RNAs in animal development. Nat Rev Mol Cell Biol 9(3):219-230.

264. He L \& Hannon GJ (2004) MicroRNAs: small RNAs with a big role in gene regulation. (Translated from eng) Nat Rev Genet 5(7):522-531 (in eng).

265. Esquela-Kerscher A \& Slack F (2006) Oncomirs [mdash] microRNAs with a role in cancer. Nat Rev Cancer 6:259 - 269.

266. Gilhuis $\mathrm{HJ}$, et al. (2000) Comparative genomic hybridization of medulloblastomas and clinical relevance: eleven new cases and a review of the literature. (Translated from eng) Clin Neurol Neurosurg 102(4):203-209 (in eng).

267. Stearns D, et al. (2006) c-myc overexpression causes anaplasia in medulloblastoma. Cancer Res 66(2):673 - 681.

268. Lamont JM, McManamy CS, Pearson AD, Clifford SC, \& Ellison DW (2004) Combined Histopathological and Molecular Cytogenetic Stratification of Medulloblastoma Patients. Clinical Cancer Research 10(16):5482-5493.

269. Badiali $\mathrm{M}$, et al. (1991) $\mathrm{N}$-myc and c-myc oncogenes amplification in medulloblastomas. Evidence of particularly aggressive behavior of a tumor with c-myc amplification. (Translated from eng) Tumori 77(2):118-121 (in eng).

270. Aldosari N, et al. (2000) Characterization of chromosome 17 abnormalities in medulloblastomas. Acta Neuropathologica 99(4):345-351.

271. Saheki A, Terasaki T, Tamai I, \& Tsuji A (1994) \&lt;i\&gt;In Vivo\&lt;/i\&gt; and \&lt;i\&gt;in Vitro\&lt;/i\&gt; Blood-Brain Barrier Transport of 3-Hydroxy-3-Methylglutaryl Coenzyme A (HMG-CoA) Reductase Inhibitors. Pharmaceutical Research 11(2):305-311.

272. Kuang SQ, et al. (2008) Genome-wide identification of aberrantly methylated promoter associated CpG islands in acute lymphocytic leukemia. Leukemia 22(8):1529-1538. 
273. Hermeking $\mathrm{H}$, et al. (2000) Identification of CDK4 as a target of C-MYC. Proceedings of the National Academy of Sciences 97(5):2229-2234.

274. Marcelli M, et al. (1998) Caspase-7 Is Activated during Lovastatin-induced Apoptosis of the Prostate Cancer Cell Line LNCaP. Cancer Research 58(1):76-83.

275. Ukomadu C \& Dutta A (2003) p21-dependent Inhibition of Colon Cancer Cell Growth by Mevastatin Is Independent of Inhibition of G1 Cyclin-dependent Kinases. Journal of Biological Chemistry 278(44):43586-43594.

276. O'Donnell MP, Kasiske BL, Kim Y, Atluru D, \& Keane WF (1993) The mevalonate pathway: importance in mesangial cell biology and glomerular disease. (Translated from eng) Miner Electrolyte Metab 19(3):173-179 (in eng).

277. Logette E, et al. (2005) Caspase-2, a Novel Lipid Sensor under the Control of Sterol Regulatory Element Binding Protein 2. Mol. Cell. Biol. 25(21):9621-9631. 


\section{Appendix II}

Abbr - Full

3'UTR - 3' Untranslated Region

APC - Polyposis Coli

AR -Average Risk

bHLHZip - Basic Helix-Loop-Helix Leucine Zipper

CDC25A - Cell Division Cycle 25A

CDK - Cyclin Dependent Kinase

$\mathrm{CGH}$ - Comparative Genomic Hybridization

CK1 $\alpha$ - Casein Kinase $1 \alpha$

CLL - Chronic Lymphocytic leukemia

CNS - Central Nervous System

CRT - Conformal Radiation Therapy

CSC - Cancer Stem Cell

CSRT - Craniospinal Radiatherapy

CT - CAT scan

Dsh - Dishevelled

E2F $1 / 2$ - E2 promoter binding Factor $1 / 2$

EGFR -Epidermal Growth Factor Receptor

EGL - External granular layer

ES - Embryonic Stem cells 
FBW7 - F-box and WD repeat domain containing 7

GFP - Greem Fluorescent Protein

GSK-3 $\beta$ - Glycogen Synthase Kinase - $3 \beta$

HAT - Histone acetyl Transferase

HGF - Hepatocyte Growth Factor

HGMA2 - high mobility group AT-hook 2

HIF-1 - Hypoxia-Inducible Factor !

HR - High Risk

HSC - haematopoietic Stem cell

IGFR - Insulin-like Growth Factor Receptor

IQ -Intelligence Quotient

LA/C - Large/anaplastic medulloblastoma

LDL - Low density lipoprotein

LR - Low risk

LRP - LDL Receptor-related Protein

MB -Myc-Box

MBEN-Medulloblastoma with extensive nodularity

miRNA - MicroRNA

MRI - Magnetic resonance Imaging

Mut - Mutant

ncRNA - Non-coding RNA

NSC - Neural Stem Cell

nt - Nucleotide 
ODC - Ornithine Decarboxylase

PDCD4 - programmed Cell Death 4

PDGFR - Platelet-derived growth Factor receptor

PI3K - Phosphatidylinositol-3-kinase

PNET - Primitive neuroectodermal tumor

PNK -Polynucleotide Kinase

RB - Retinoblastoma

RFP (mKate) - Red Fluorescent Protein

RISC - RNA-Induced Silencing Complex

ROS -Reactive Oxygen Species

RTK - Receptor Tyrosine Kinase

$\mathrm{SC}-$ Stem cell

Shh - Sonic Hedgehog

SREBF1 - Sterol Regulatory Element Binding Transcription Factor 1

TAD - Transactivation domain

Tcf/Lef - T-cell factor/lef

TGF $\beta$ - Transforming Growth Factor $\beta$

TIC - Tumor Initiating Cell

TRK - Tyrosine Kinase

VEGF - Vascular endothelial Growth Factor

WHO - World Health Organization

Wnt - wingless

WT -Wild type 
ZEB - Zinc finger E-box binding Factor 
Department of Biochemistry and Molecular Biology,

University of Louisville,

319 Abraham Flexner Way, HSC A Rm 714

Louisville, KY 40202

(502) 802-9437

Ltapan01@louisville.edu

Place and date of birth:

Yaoundé, Central Province, Cameroon

$20^{\text {th }}$ September 1980

\section{Visa Status:}

F1 Visa

\section{Education \& training:}

Ph.D., Biochemistry and Molecular Biology, University of Louisville, KY, Pending (Anticipated December 2011)

Thesis: A Statin-regulated MicroRNA Represses c-Myc Expression and Function

Supervisor: Dr. Yong Li

2003.

B.Sc., Biochemistry, Second Class upper division, University of Buea, Cameroon,

\section{TECHNICAL SKILLS}

- Techniques for microRNA studies

Luciferase assay, miRNA plasmid cloning, purification and transfection into cell lines.

- Molecular Biological Techniques

Isolation \& Quantification of Genomic DNA; PCR; RT-PCR; Primer designing; Microscopy; Cloning; Western Blot; Apoptosis assay; Flow cytometry.

- Animal Handling techniques 
Subcutaneous tumor formation techniques (subcutaneous injection of mice); monitoring of mice for tumor growth; intraperitoneal injection of mice; Basic mice breeding technique

- Bioinformatics

BLAST search; sequence alignment; identification of exon, intron, probable genes and pseudogenes.

- Biochemical \& Instrumental methods

Nanodrop, Protein Purification \& Estimation; Spectrophotometry; Gel Electrophoresis; Immobilization of Enzymes; H\&E Staining; Immunohistochemistry and immunocytochemistry Staining.

- Culture Techniques

Maintaining cell line cell culture; Bacteria cell culture.

- Computer skills

MS Word, Excel, Power point, FrontPage, Picture Manager, EndNote 9, Adode Acrobat, Photoshop

\section{Research experience}

Doctoral Research: Department of Biochemistry and Molecular Biology, University of Louisville, Louisville, KY, 2006 to present (Research advisor: Dr. Yong Li)

- Identification of microRNAs targeting MYC: Performed gene cloning, cell culture, luciferase assay, western blotting, flow cytometry and major molecular biology techniques.

- Investigated the role played by miR-33b in the development of Medulloblastoma: Western blot, flow cytometry, qRT-PCR, neurosphere formation assay, soft agar colony formation assay, In vivo tumor formation, H\&E staining, immunocytochemistry and immunohistochemistry.

- Investigate the mechanism of lovastatin anti-medulloblastoma effect through up-regulation of miR-33b expression: western blot, qRT-PCR, subcutaneous in vivo tumor formation in mice, immunohistochemistry.

- Determine microRNAs (miR-25 and miR-30d) that target p53 and their implication in cancer.

- Elucidated the reason why NFkB is specifically over-expressed in pancreatic cancer and the role played by $\mathrm{miR}-301$.

Masters Research: Department of Biochemistry, University of Buea, Cameroon 2004 - 2006 (Research advisor: Dr. Vincent Titanji) - not completed

- Purification of Onchocerciasis oxygen peroxidase for monoclonal antibody production: electrophoresis, ELISA and protein purification.

- Breeding of mice: maintaining mice, subcutaneous injection of protein antigen in adjuvant.

- Monoclonal antibody production using myeloma derived cells. 
- Study basic biochemistry and molecular laboratory techniques: western blotting, ELISA assay, etc.

\section{Research Interest}

- Explore the role played by miRNAs in cancer development.

- Elucidate the various mechanisms by which miRNAs can act both as tumor suppressors and oncogenes.

- Explore avenues by which miRNAs can act as therapeutic targets against various diseases.

- Study the signaling pathways that are activated in disease processes, especially cancer.

- Investigate ways through which these signaling pathways could be used to develop novel targeted therapies.

\section{Teaching experience}

- Teaching assistant: University of Louisville, Advance Biochemistry II (BIOC647), Spring 2008

- Teaching assistant: University of Buea 2004-2006

\section{NON-RESEARCH EXPERIENCE}

Sale person: Worked under the supervision of Dr. Nkwenti Achu (Pharmacist, Oregon State University)

- Professional Plaza pharmacy Bamenda, Cameroon - June - September 1998

- Professional Plaza pharmacy Bamenda, Cameroon - June - September 1999

- Professional Plaza pharmacy Bamenda, Cameroon - June - September 2000

- Professional Plaza pharmacy Bamenda, Cameroon - January - October 2003

\section{Diabetes educator}

- Professional Plaza pharmacy, Bamenda - work with Dr. Nkwenti Achu in the diabetes centre to give various lesson to diabetic patients $\mathbf{- 2 0 0 3 - 2 0 0 4}$

\section{Publications}

Lu Z, Li Y, Takwi A, Li B, Zhang J, Conklin DJ, Young KM, Martin R, Li Y. miR-301a as an NF-KB activator in pancreatic cancer cells. EMBO J. 2011 Jan 5;30(1):57-67.

Kumar M, Lu Z, Takwi AA, Chen W, Callander NS, Ramos KS, Young KH, Li Y. Negative regulation of the tumor suppressor p53 gene by microRNAs. Oncogene (2010), 111. 
Takwi A, Yong Li. The p53 Pathway Encounters the MicroRNA World. Curr Genomics 2009; 10(3): 194-7.

\section{Awards and Honors}

- Integrated Programs in Biomedical Sciences (IPIBS) Doctoral Fellowship, 2006 present

- American Society of Biochemistry and Molecular Biology (ASBMB) Travel Award, Anahiem, 2010

- Speaker at Rustbelt RNA meeting Ohio, 2009

- Winner of Rustbelt RNA meeting Poster competition, Ohio, 2008

- Financial Aid for outstanding performance by Ministry for Higher Education, Cameroon, 2002

- Financial Aid for outstanding performance by Ministry for Higher Education, Cameroon, 2001 